\title{
Study and Implementation of Lower-Ground CPW Transmission Lines for the Design of V-band Si-based Transceiver Blocks
}

\author{
by \\ Ibrahim Haroun, B.Sc., M.Sc., B.Ed. \\ Thesis submitted to the Faculty of Graduate Studies and Research in \\ partial fulfillment of the requirements for the degree of
}

\author{
Doctor of Philosophy \\ in
}

Electrical Engineering

Ottawa-Carleton Institute of Electrical and Computer Engineering Department of Electronics

Carleton University

March 2010

Copyright $(\underset{2}{ } 2010$, Ibrahim Haroun 


\begin{tabular}{|c|c|}
\hline $\begin{array}{l}\text { Library and Archives } \\
\text { Canada }\end{array}$ & $\begin{array}{l}\text { Bibliotheque et } \\
\text { Archives Canada }\end{array}$ \\
\hline $\begin{array}{l}\text { Published Heritage } \\
\text { Branch }\end{array}$ & $\begin{array}{l}\text { Direction du } \\
\text { Patrimoine de l'édition }\end{array}$ \\
\hline $\begin{array}{l}395 \text { Wellington Street } \\
\text { Ottawa ON K1A ON4 } \\
\text { Canada }\end{array}$ & $\begin{array}{l}\text { 395, rue Wellington } \\
\text { Ottawa ON K1A ON4 } \\
\text { Canada }\end{array}$ \\
\hline
\end{tabular}

Your file Votre référence

ISBN: 978-0-494-67891-6

Our file Notre référence

ISBN: 978-0-494-67891-6

NOTICE:

The author has granted a nonexclusive license allowing Library and Archives Canada to reproduce, publish, archive, preserve, conserve, communicate to the public by telecommunication or on the Internet, loan, distribute and sell theses worldwide, for commercial or noncommercial purposes, in microform, paper, electronic and/or any other formats.

The author retains copyright ownership and moral rights in this thesis. Neither the thesis nor substantial extracts from it may be printed or otherwise reproduced without the author's permission.
AVIS:

L'auteur a accordé une licence non exclusive permettant à la Bibliothèque et Archives Canada de reproduire, publier, archiver, sauvegarder, conserver, transmettre au public par télécommunication ou par l'Internet, prêter, distribuer et vendre des thèses partout dans le monde, à des fins commerciales ou autres, sur support microforme, papier, électronique et/ou autres formats.

L'auteur conserve la propriété du droit d'auteur et des droits moraux qui protège cette thèse. Ni la thèse ni des extraits substantiels de celle-ci ne doivent être imprimés ou autrement reproduits sans son autorisation.
In compliance with the Canadian Privacy Act some supporting forms may have been removed from this thesis.

While these forms may be included in the document page count, their removal does not represent any loss of content from the thesis.
Conformément à la loi canadienne sur la protection de la vie privée, quelques formulaires secondaires ont été enlevés de cette thèse.

Bien que ces formulaires aient inclus dans la pagination, il n'y aura aucun contenu manquant.

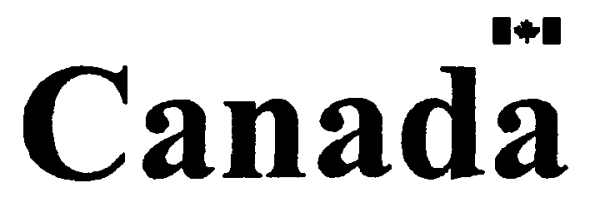




\section{Abstract}

The demand for ultra-high data-rate wireless communication systems and the availability of $7 \mathrm{GHz}$ of unlicensed bandwidth in the $60 \mathrm{GHz}$ ISM band (57-64 GHz) as well as the advances in the CMOS technology, have created a substantial interest in the development of small-size, low-cost millimetre-wave (mmW) systems operating at frequencies in the V-band (50-75 GHz). Although CMOS technology enables having a low-cost system-on-chip that integrates both the analog RF and the digital circuits with the mmW circuits into a single-chip, the technology has design challenges because of its lossy silicon substrate and its process design rules restrictions.

This thesis explores the use of the proposed lower-ground coplanar waveguide (LG-CPW) transmission lines to overcome the limitations of the CMOS technology design rules, which limit the maximum/minimum metal width and the minimum spacing between metal strips. The proposed transmission lines enable the realization of a wide range of impedances that facilitates the optimization and design of V-band passive and active sub-systems without violating the design rules of the CMOS process. Also, they enable achieving low loss transmission line structures.

As applications for the implementation of these LG-CPW transmission lines, two key RF building blocks including a $60-\mathrm{GHz}$ band quadrature branch-line coupler and 
a V-band low noise amplifier have been designed and implemented in a 90-nm RF CMOS process.

The low noise amplifier block utilized LG-CPW transmission lines as matching elements to enable the realization of the needed impedances for achieving optimal noise performance. To the author's knowledge, the amplifier of this thesis is one of the lowest noise figure and the highest gain that have been achieved with a 90$\mathrm{nm}$ CMOS technology in the V-band. Also, the power consumption is among the lowest power consumptions of $\mathrm{V}$-band CMOS low noise amplifiers.

The design of the coupler block utilized capacitively loaded LG-CPW transmission lines to reduce the physical size of the quarter-wavelength elements of the coupler. The coupler's size is $0.102 \mathrm{~mm}^{2}$ which is more than $75 \%$ size reduction compared to conventional CPW branch-line couplers. 


\section{Acknowledgements}

Many people have contributed in important ways to this thesis. The support and guidance of my advisors, Professors Jim Wight and Calvin Plett have made my graduate studies at the Department of Electronics at Carleton University rewarding. I am also grateful for the kind support of Blazenka Power and Anna Lee from the Department of Electronics.

My family has also played a key role throughout the years supporting me always. Special thanks to my wife Magdalene, my sons Adam and Andrew, and my daughter Karena.

I must also greatly thank Dr. Valek Szwarc the manager of the MMIC group at the Communications Research Centre Canada (CRC) for his support and encouragement. Also thanks to Audrey Honeywell for administrative helps at CRC; and Dr. Gerry Chan, former vice president at CRC, who was instrumental to my trip to the Chip Implementation Center (CIC) in Taiwan.

I would like to extend my thanks to my colleagues at CRC Dr. Khelifa Hettak and Dr. Rony Amaya for thoughtful discussions. Special thanks to Dr. Da-Chiang Chang and his team at $\mathrm{CIC}$ for their support with the measurements during my visit to Taiwan. 
Finally, thanks to my former colleagues and friends Abdel-Kader Dhouib, Seif Karim, Terry Kenny, George Khoury, and Andy Rowland who have always been supportive. 


\section{Author's Publications}

The following papers were published and presented as a direct result of this work.

\section{i) Journal Papers:}

1. Haroun, I., Wight, J., Plett, C., et al.," Experimental Analysis of a $60-\mathrm{GHz}$ Compact EC-CPW Branch-Line Coupler for mm-Wave CMOS Radios," accepted for publication in IEEE Microwave and Wireless Components Letters.

\section{ii) Conference Papers:}

2. Haroun, I., Wight, J., Plett, C., et al., "A V-band 90-nm CMOS Low-Noise Amplifier with Modified CPW Transmission Lines for UWB Systems," IEEE Radio and Wireless Symposium (RWS-2010), New Orleans, January 2010.

3. Haroun, I., Wight, J., Plett, C., et al., "A V-band 90-nm CMOS Low-Noise Amplifier with Modified CPW Transmission Lines for UWB Systems," Invited Paper, 2010 IEEE Symposium on SiRF, New Orleans, January 2010.

4. Haroun, I., Wight, J., Plett, C., et al., "Multi-band $700 \mathrm{MHz} / 2.4 \mathrm{GHz} / 60 \mathrm{GHz} \mathrm{RF}$ Front-End for Radio-over-Fiber Base-Stations," IEEE Radio and Wireless Symposium (RWS-2010), New Orleans, January 2010.

5. Haroun, I., Hsu, Y-C., Wight, J., Plett, C., "A CMOS Low-Noise Amplifier with VPW Matching Elements for 60-GHz-band Gbit/s Wireless Systems," 2009 Asia Pacific Microwave Conference, Singapore, December 2009.

6. Haroun, I., Wight, J., Plett, C., et al.," Experimental Characterization of ECCPW Transmission Lines and Passive Components for 60-GHz CMOS Radios," accepted for publication in IEEE MTT-S 2010 International Microwave Symposium, May 23-28, 2010. 


\section{Table of Contents}

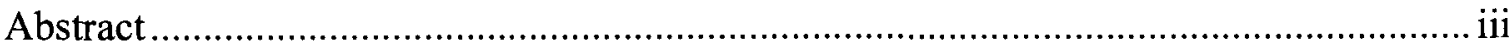

Acknowledgements .............................................................................................. V

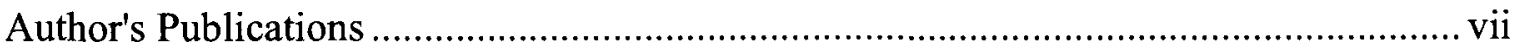

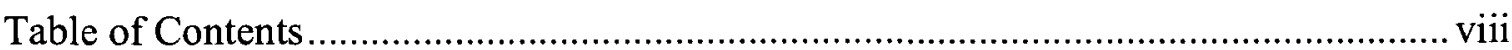

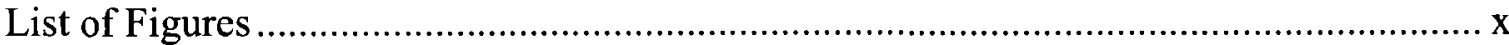

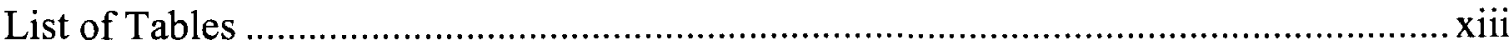

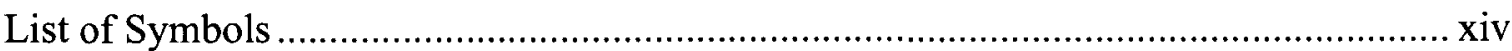

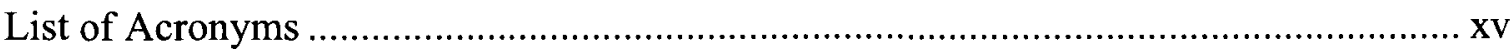

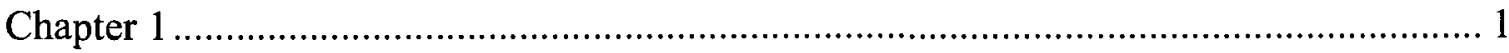

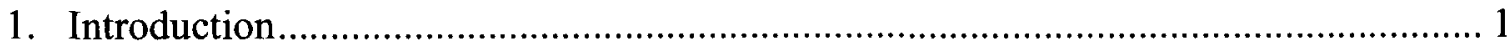

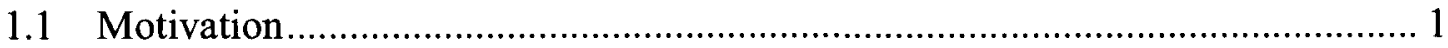

1.2 Applications of Transmission Lines in mmW Radio Front-Ends....................... 4

1.3 CPW Transmission Lines in Silicon Technology ......................................... 6

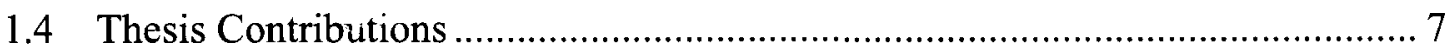

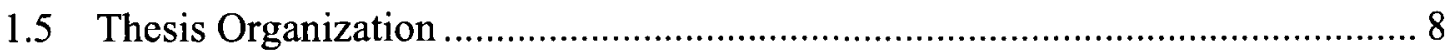

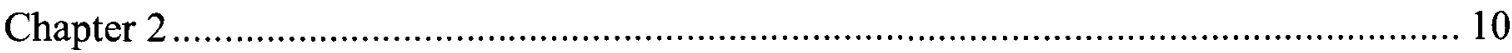

2. Transmission Lines in mmW Radio Front-Ends .................................................. 10

2.1 Radio Transceiver Front-Ends .................................................................... 10

2.2 State-of-the-art mmW Transmission Lines................................................... 13

2.3 Analysis of mmW Transmission Lines......................................................... 15

2.3.1. Maxwell's Electromagnetic Field Equations ........................................ 15

2.3.2. Helmholtz Equation ....................................................................... 16

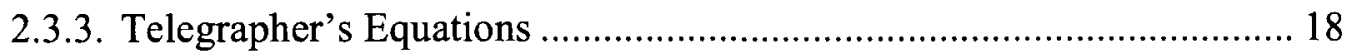

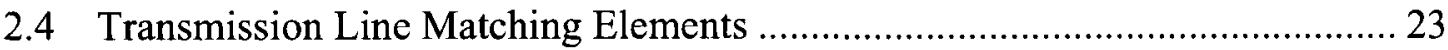

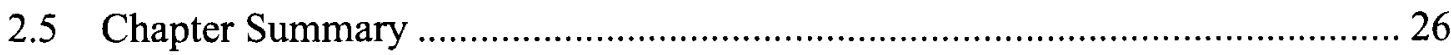

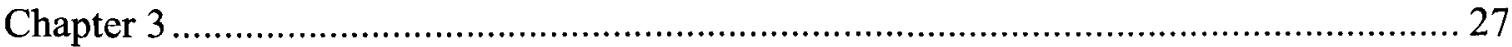

3. Millimeter-wave Si-based Lower-Ground CPW Transmission Lines......................... 27

3.1 Transmission Lines in Silicon Technology ................................................. 27

3.2 Silicon-based CPW Transmission Lines........................................................ 30

3.3 Si-based Lower-Ground CPW Transmission Lines......................................... 31

3.4 Analysis of Si-based LG-CPW Transmission Lines.......................................... 34 
3.5 Extraction of Measured Parameters using Chain Matrix De-embedding 38

3.6 Measurements and EM Simulation Results for CMOS LG-CPW

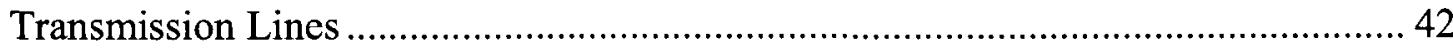

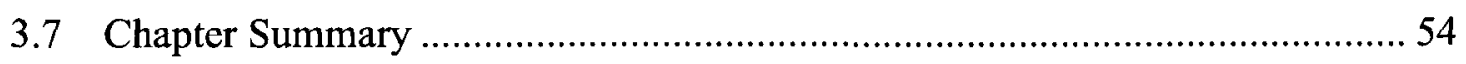

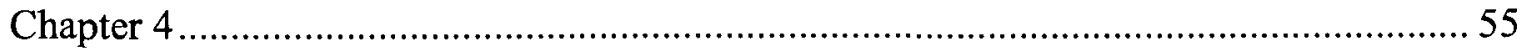

4. Design of a V-band Si-based LNA using LG-CPW Transmission Lines................. 55

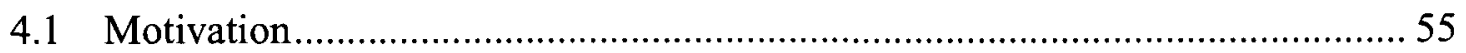

4.2 Noise Figure of a Low Noise Amplifier ..................................................... 58

4.3 Impact of the Transistor Size on Noise-Figure ........................................... 59

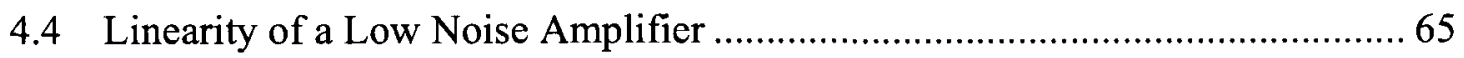

4.5 Design of a V-band LNA with LG-CPW Transmission Lines ......................... 68

4.6 Layout of the Amplifier's Matching Networks ............................................ 76

4.7 Experimental and Post-layout Simulation Results....................................... 78

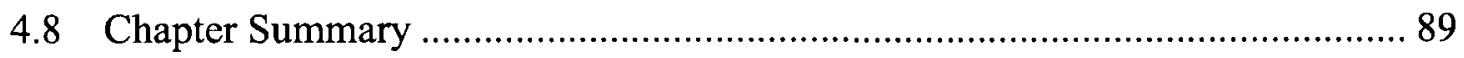

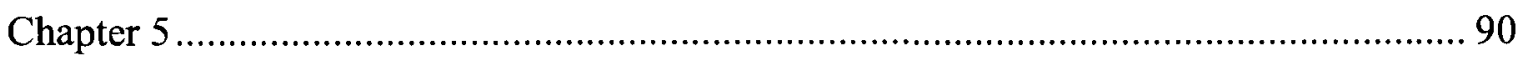

5. Design of a V-band CMOS Reduced-Size LG-CPW $90^{\circ}$ Coupler .......................... 90

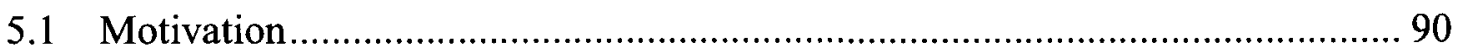

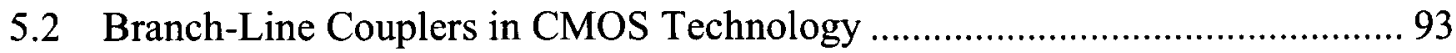

5.2.1 CMOS Capacitively Loaded LG-CPW Transmission Lines ................ 94

5.3 Analysis of Reduced-Size Transmission Lines........................................... 95

5.4 Design of a Reduced-Size $90^{\circ}$ Coupler using CMOS LG-CPW Elements ....... 97

5.5 Layout of the CMOS LG-CPW 90 ${ }^{\circ}$ Branch-Line Coupler ............................ 102

5.6 Design Flow of a CMOS LG-CPW Branch Line Coupler ............................. 104

5.7 Experimental and Post-layout Simulation Results.................................... 105

5.8 Chapter Summary ...................................................................... 110

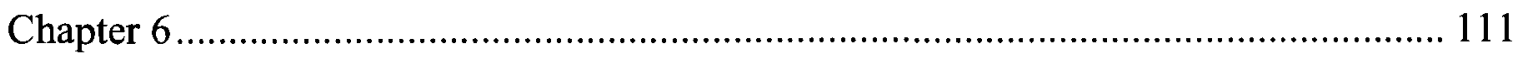

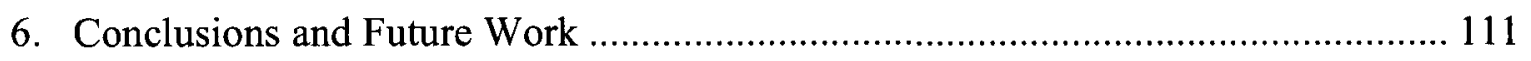

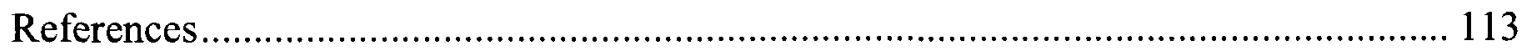




\section{List of Figures}

Figure 1.1: Worldwide $60 \mathrm{GHz}$ allocated frequency band

Figure 1.2: Potential applications for $60 \mathrm{GHz}$ high-speed short-range wireless

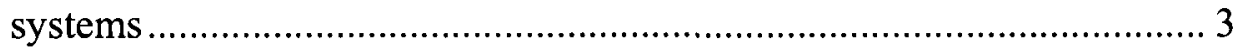

Figure 1.3: $\mathrm{mmW}$ Radio-Over-Fiber sensor network ............................................... 4

Figure 1.4: Applications of transmission line elements in mmW building blocks (a) Low-noise amplifier, (b) Quadrature branch-line coupler. (c) Low pass filter, (d) Wilkinson power splitter.

Figure 1.5: Microphotograph of $\mathrm{mmW}$ radio front-end building blocks (this work) 5

Figure 1.6: Cross-section view of a Si-based CPW transmission line......................... 6

Figure 2.1: Block diagram of mmW Radio Front-End transceiver ......................... 11

Figure 2.2: Cross-section of $\mathrm{mmW}$ planar transmission Lines (a) Microstrip,

(b) Coplanar waveguide, (c) Conductor-backed CPW, (d) Strip line.13

Figure 2.3: Circuit model of a transmission line section of a length $\mathrm{dz}$.................. 18

Figure 2.4: Loaded transmission line................................................................ 23

Figure 3.1: Cross-section view of the metal layers in a typical RF-CMOS process with 9-metal layers........................................................................... 28

Figure 3.2: Cross-section view of a Si-based microstrip line and its electric field distribution.

Figure 3.3: CMOS CPW Structure (a) 3-D view (Si-substrate is not shown), (b)

Electric field distribution 30

Figure 3.4: CMOS LG-CPW transmission line structure (a) 3D view, (b) Cross section. 32

Figure 3.5: 3-D view and a cross section of a vertical LG-CPW transmission line 33

Figure 3.6: Equivalent circuit model of a CMOS LG-CPW transmission line section $\Delta l$ (a) Detailed lumped-element model (b) Simplified equivalent model

Figure 3.7: Two identical CPW test structures of different lengths for extracting the $A B C D$ parameters of an embedded CPW transmission line. .................. 38

Figure 3.8: Representation of the long CPW test structure ....................................... 39

Figure 3.9: Characteristic impedances of CMOS CPW transmission lines of different

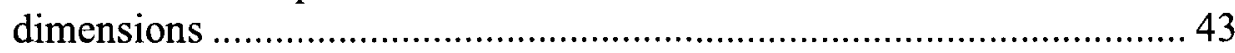

Figure 3.10: Resistance per unit-length for two 50 CMOS CPW transmission lines of

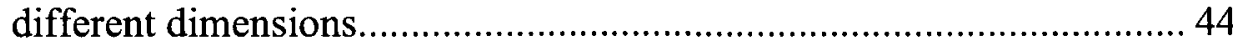

Figure 3.11: Attenuation factor of two CPWs with different dimensions ................. 45

Figure 3.12: Microphotograph of short and long CPW test structures implemented on metal-9 in a $90 \mathrm{~nm} 9$-metal layers CMOS process. 45

Figure 3.13: Extracted and simulated characteristic impedances of a CMOS CPW transmission line on $\mathrm{M} 9(\mathrm{~W}=10 \mu \mathrm{m}, \mathrm{S}=2.5 \mu \mathrm{m}, \mathrm{G}=12 \mu \mathrm{m}, \mathrm{t}=0.81 \mu \mathrm{m}) 46$

Figure 3.14: Extracted and simulated attenuation of a $50 \mathrm{Ohms} \mathrm{CPW}$ transmission line on $\mathrm{M} 9(\mathrm{~W}=10 \mu \mathrm{m}, \mathrm{S}=2.5 \mu \mathrm{m}, \mathrm{G}=12 \mu \mathrm{m}, \mathrm{t}=0.81 \mu \mathrm{m})$. 
Figure 3.15: Extracted and simulated effective permittivity of a $50 \mathrm{Ohms}$ CMOS CPW transmission line on $\mathrm{M} 9(\mathrm{~W}=10 \mu \mathrm{m}, \mathrm{S}=2.5 \mu \mathrm{m}, \mathrm{G}=12 \mu \mathrm{m}$, $\mathrm{t}=0.81 \mu \mathrm{m})$

Figure 3.16: Characteristic impedances of LG-CPW transmission lines of different heights.

Figure 3.17: Extracted and simulated characteristic impedances of CMOS LG-CPW

and CPW transmission lines

Figure 3.18: Extracted attenuation/mm of CMOS LG-CPW and CPW transmission lines.

Figure 3.19: Extracted effective permittivity of CMOS LG-CPW and CPW transmission lines

Figure 3.20: Microphotograph of short and long CPW and LG-CPW test structures implemented in a 90nm 9-metal layers CMOS process.

Figure 3.21: Simulated extreme characteristic impedance of LG-CPW in a $90-\mathrm{nm}$ CMOS process

Figure 4.1: A simplified block diagram of a $60 \mathrm{GHz}$ band Radio-over-Fiber transceiver front-en......

Figure 4.2: Minimum noise figure vs. the number of fingers for three different finger widths $\left(\mathrm{V}_{\mathrm{GS}}=0.7 \mathrm{~V}, \mathrm{~V}_{\mathrm{DS}}=1.5 \mathrm{~V}\right)$ at $57 \mathrm{GHz}$

Figure 4.3: Equivalent noise resistance vs. number of fingers $\left(\mathrm{W}_{\mathrm{f}}=1 \mathrm{um}, \mathrm{V}_{\mathrm{GS}}=0.7 \mathrm{~V}\right.$, $\mathrm{V}_{\mathrm{DS}}=1.5 \mathrm{~V}$ ) at $57 \mathrm{GHz}$.

Figure 4.4: $\mathrm{NF}_{\min } \mathrm{vs}$. frequency for different device finger widths $\left(\mathrm{V}_{\mathrm{GS}}=0.7 \mathrm{~V}\right.$, $\left.\mathrm{V}_{\mathrm{DS}}=1.5 \mathrm{~V}\right)$

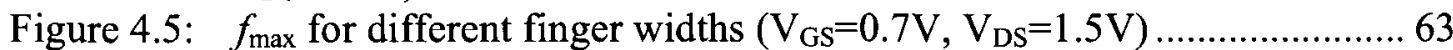

Figure 4.6: I-V Curves of the $30 \mathrm{x} 1 \mu \mathrm{m} / 0.09 \mu \mathrm{m}$ nMOS device..................................64

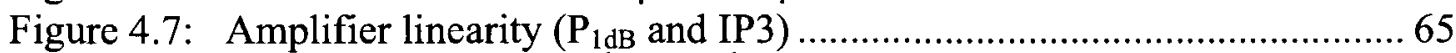

Figure 4.8: Output spectrum of $2^{\text {nd }}$ and $3^{\text {rd }}$ order two-tone inter-modulation products

Figure 4.9: Cascode Amplifier Configuration 66

Figure 4.10: Test bench for simulating the core cascode amplifier 68

Figure 4.11: Simulated $\mathrm{NF}_{\min }$ and $\mathrm{G}_{\max }$ of the core cascode $\mathrm{w} / \mathrm{o}$ matching $(\mathrm{Q} 1$ is

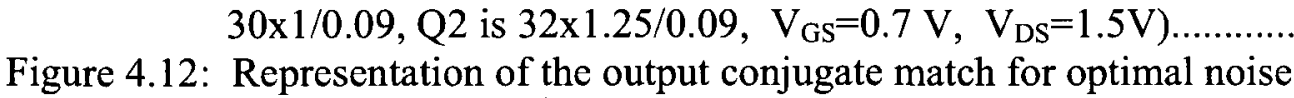
performance $\left(\Gamma_{\text {out }}\right)^{*}=\Gamma_{\mathrm{ML}}, \Gamma_{\mathrm{MS}}=\Gamma_{\mathrm{opt}}$ 71

Figure 4.13: Circuit schematic of a V-band single-stage amplifier with ideal transmission line matching-elements.

Figure 4.14: Simulated NF and gain of the single-stage amplifier of ideal TL matching-elements

Figure 4.15: Simulated input and output return losses of the single-stage amplifier 74

Figure 4.16: Design flow of a LG-CPW Low Noise Amplifier ................................. 75

Figure 4.17: 3-D View (Si-substrate is not shown) of the input and output matching networks with LG-CPW transmission line elements.

Figure 4.18: Photograph of the 90-nm CMOS $57 \mathrm{GHz}$ single-stage amplifier of this work (chip area is $0.2 \mathrm{~mm} 2$ ) .

Figure 4.19: Block diagram of the noise figure test setup for characterizing the single-stage LG-CPW LNA 
Figure 4.20: V-band test setup at the Chip Implementation Centre in Taiwan .......... 80

Figure 4.21: Measured and post-layout simulated NF of the CMOS LG-CPW singlestage amplifier $(\mathrm{Vdd}=1.5 \mathrm{~V}, \mathrm{Idd}=11 \mathrm{~mA})$

Figure 4.22: Measured and post-layout simulated gain of the CMOS EC-CPW single-stage amplifier (Vdd $=1.5 \mathrm{~V}, \mathrm{Idd}=11 \mathrm{~mA}$ )

Figure 4.23: Measured and post-layout simulated input return losses of the CMOS LG-CPW single-stage amplifier $(\mathrm{Vdd}=1.5 \mathrm{~V}, \mathrm{Idd}=11 \mathrm{~mA})$

Figure 4.24: Measured and post-layout simulated S22, and simulated S22 with modified transistor model of the CMOS LG-CPW single-stage amplifier

Figure 4.25: Test setup for measuring the gain and return losses of the single-stage

LNA chip.

Figure 4.26: Measured $\mathrm{IP}_{3}$ of a $57 \mathrm{GHz}$ EC-CPW Single-Stage Amplifier with 100

MHz Tone Spacing.

Figure 4.27: Test set up for measuring the third-intercept point of the single-stage amplifier.

Figure 5.1: A simplified block diagram of a V-band Radio-Over-Fiber transceiver front-end for high-speed wireless applications ................................. 92

Figure 5.2: A conventional branch-line coupler ................................................. 94

Figure 5.3: Capacitively loaded CMOS LG-CPW transmission line element......... 95

Figure 5.4: Schematic representation of (a) conventional transmission line section,

(b) capacitively loaded line...

Figure 5.6: Simulated S-parameters of the branch-line coupler (ideal transmission lines and lumped capacitors).

Figure 5.7: Simulated phase difference of the branch-line coupler (ideal transmission lines and lumped capacitors). 101

Figure 5.8: 3-D view (Si-substrate is not shown) of the layout of the $60 \mathrm{GHz}$ branch-line coupler.... 103

Figure 5.9: Microphotograph of the fabricated $60 \mathrm{GHz}$ CMOS reduced-size coupler

Figure 5.10: Design flow of the ED-CPW Low Noise Amplifier 103

Figure 5.11: Measured and post-layout simulated S-parameters of the LG-CPW

Figure 5.12: Measured and post-layout simulated phase-difference of the LG-CPW 104 branch line coupler.

Figure 5.13: Measured and post-layout simulated amplitude-imbalance of the LG-

CPW branch line coupler.... 108

Figure 5.14: Measured and post-layout simulated return loss of the EC-CPW branch line coupler. 


\section{List of Tables}

Table 3-1: Dimensions of conventional CPW transmission line 42

Table 4-1: Performance summary of this single-stage amplifier in comparison with V-band $90 \mathrm{~nm}$ CMOS amplifiers. 


\section{List of Symbols}

$\begin{array}{ll}\varepsilon_{0} & \text { Permittivity of Vacuum }\left(8.854 \times 10^{-12} \mathrm{~F} / \mathrm{m}\right) \\ \varepsilon_{\mathrm{r}} & \text { Relative Permittivity (dielectric constant) } \\ \mu_{\mathrm{o}} & \text { Magnetic Permeability of the Vacuum }\left(4 \pi \times 10^{-7} \mathrm{H} / \mathrm{m}\right) \\ \mu_{\mathrm{r}} & \text { Relative Permeability of the Medium } \\ \sigma & \text { Electric Conductivity of the Medium } \\ \rho_{v} & \text { Volume Charge Density } \\ \delta & \text { Skin depth } \\ \gamma & \text { Propagation Constant } \\ \alpha & \text { Attenuation Factor } \\ \beta & \text { Phase of Propagation Constant } \\ \lambda & \text { Free space wavelength in meters } \\ \lambda_{\mathrm{g}} & \text { Guided wavelength in meters } \\ \mathrm{v}_{\mathrm{p}} & \text { phase velocity }\end{array}$




\section{List of Acronyms}

\begin{tabular}{|c|c|}
\hline BS & Base-station \\
\hline B & Magnetic Flux Density \\
\hline CMOS & Complementary Metal-Oxide Semiconductor \\
\hline CPW & Coplanar Wave-guide \\
\hline $\mathrm{D}$ & Electric Flux Density \\
\hline DSP & Digital Signal Processing \\
\hline $\mathrm{E}$ & Electric Field Intensity \\
\hline EM & Electromagnetic \\
\hline $\mathrm{G}_{\max }$ & Maximum Available Gain \\
\hline $\mathrm{H}$ & Magnetic Field Intensity \\
\hline ISM & Industrial, Science, and Medical \\
\hline $\mathrm{J}$ & Current Density \\
\hline LG-CPW & Lower-ground CPW \\
\hline LNA & Low Noise Amplifier \\
\hline $\mathrm{NF}_{\min }$ & Minimum Noise Figure \\
\hline $\mathrm{mmW}$ & Millimeter-wave \\
\hline nMOS & n-type Metal-Oxide Semiconductor \\
\hline Q & Quality factor \\
\hline RF & Radio Frequency \\
\hline $\mathrm{Si}$ & Silicon \\
\hline TEM & Transverse Electric Magnetic \\
\hline $\mathrm{V}_{\mathrm{G}}$ & Gate Bias Voltage \\
\hline$V_{D}$ & Drain Bias Voltage \\
\hline WLAN & Wireless Local Area Network \\
\hline WPAN & Wireless Personal Area Network \\
\hline
\end{tabular}




\section{Chapter 1}

\section{Introduction}

\subsection{Motivation}

The demand for ultra-high speed wireless communication systems is increasing daily with the emergence of a multitude of multimedia applications. In particular, the need for high speed personal internet access, video streaming, point-to-point or point-tomultipoint wireless data links, and broadband wireless sensor networks. This demand has created substantial interest in utilizing millimetre-wave $(\mathrm{mmW})$ frequencies in the $\mathrm{V}$ band for these applications [1-4]. Particularly, the $60 \mathrm{GHz}$ unlicensed ISM band (57-64 $\mathrm{GHz}$ ) is of much interest [5-6]. Figure 1.1 shows the worldwide $60 \mathrm{GHz}$ allocated frequency band.

Frequeny A llocated in $\mathrm{GHz}$

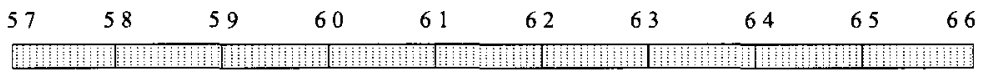

A usralia
Canada and
USA
Japan
Europe

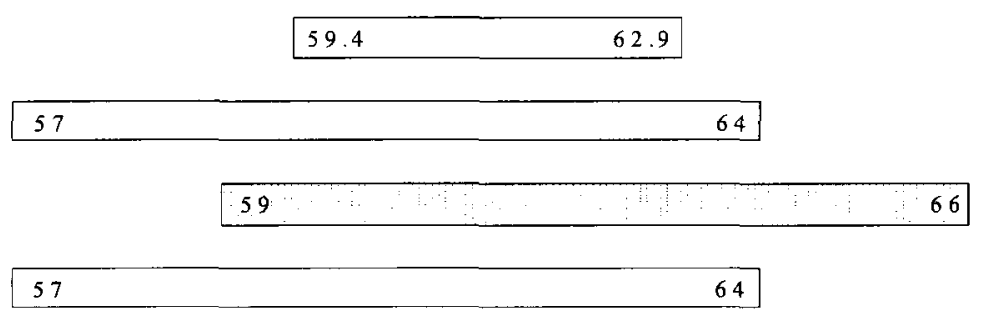

Figure 1.1: Worldwide $60 \mathrm{GHz}$ allocated frequency band 
The main reasons for the interest in the $60 \mathrm{GHz}$ band include: the availability of 7 GHz license-free bandwidth, the advances in silicon (Si) technology [7] which facilitates full integration of the RF and the digital circuitry into a single chip; the small size of the antennas at $60 \mathrm{GHz}$ which enable the implementation of multiple antenna phased-array systems; the ability to implement frequency reuse cellular networks at $60 \mathrm{GHz}$ to increase the system capacity; the oxygen absorption around $60 \mathrm{GHz}$ which limits long-distance interference; the potential for transmitting high data rate in the range of Giga-bits/s.

The recently developed IEEE $802.15 .3 \mathrm{c}$ standards for $\mathrm{mmW}$ wireless personal area networks (WPAN) aim to deliver data rates of 2 to $3 \mathrm{Gbit} / \mathrm{s}$ or even more. This data rate is much higher than the data-rate of the current WLAN systems [8] in the $2.4-2.48 \mathrm{GHz}$ and $5.15-5.825 \mathrm{GHz}$ bands. The $60 \mathrm{GHz}$ band is well suited for short-range high-speed wireless applications including personal area networking (WPAN) in home/office environments and radio-over-fiber sensor network [9]-[10]. Figure 1.2 shows potential applications for using the $60 \mathrm{GHz}$ band in an indoor environments, and Figure 1.3 shows a potential application for $\mathrm{mmW}$ radio-over-fiber sensor network.

In $\mathrm{mmW}$ radio front-ends of wireless systems, transmission lines are indispensable components because of their versatile use as filters, matching networks, phase shifters, antenna feed networks, power splitters/combiners, couplers, balanced mixers, balanced amplifiers, and interconnects. Therefore, the transmission line elements of the radio frontends influence the system performance. Until recently passive circuit-elements played a relatively limited role in Si-based integrated circuits in comparison with the active devices; this is because of the lossy silicon substrate and the relatively large size of the 
passive components, as well as the geometry limitations imposed by the silicon CMOS design rules. These design issues and the demand for low-power consumption, high datarate, and low bit-error-rate radio systems have led to a surge of interest in reduced-size on-chip passive circuit elements. In this thesis, the focus will be placed on the analysis and design of Si-based multilayer transmission lines which overcome the limitations of the CMOS design rules. The focus of this study is also placed on the utilization of the proposed transmission lines to develop a V-band CMOS low noise amplifier, and a reduced-size $60-\mathrm{GHz}$ CMOS quadrature coupler.
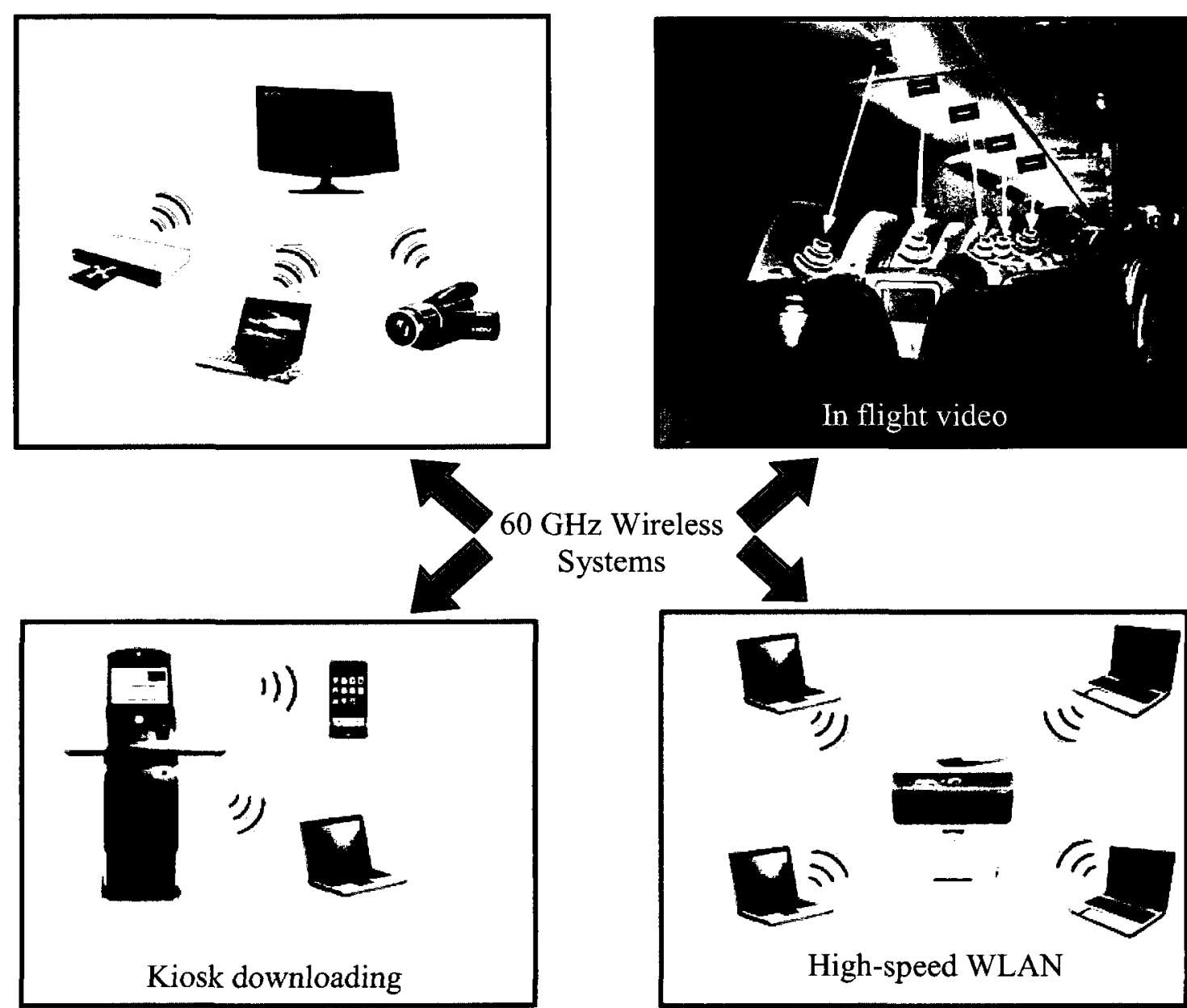

\section{GHz Wireless \\ Systems}
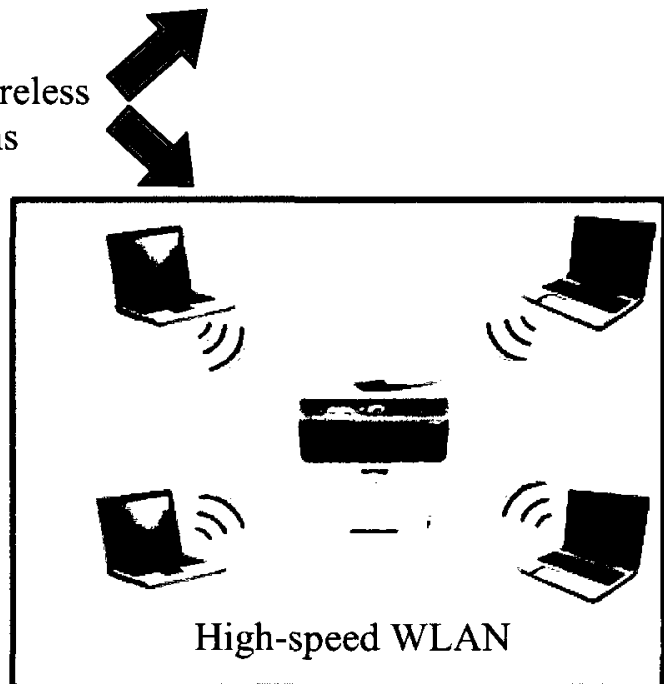

Figure 1.2: Potential applications for $60 \mathrm{GHz}$ high-speed short-range wireless systems 


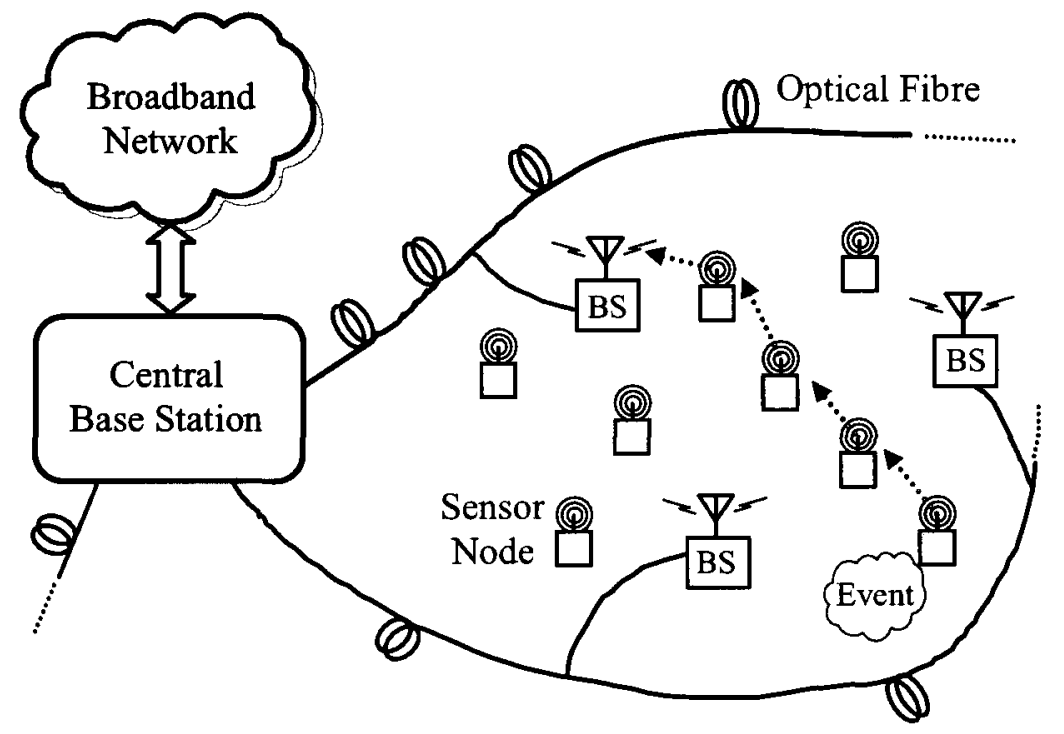

Figure 1.3: mmW Radio-Over-Fiber sensor network

\subsection{Applications of Transmission Lines in $\mathbf{m m W}$ Radio Front-Ends}

Transmission line elements are used in many building blocks of mmW radio front-ends in wireless systems. Figure 1.4 shows some radio frequency (RF) building blocks based on transmission lines. In Figure 1.4 (a), the input and output impedance matching networks of the amplifier are implemented using transmission line elements with short-circuited stubs. Impedance matching enables having minimum noise figure, maximum gain, and minimum reflections. Figure 1.4 (b) shows a quadrature branch-line coupler where the coupler's elements are implemented with quarter-wave transmission lines. Figure 1.4 (c) shows a low pass filter where the filter's series elements are implemented with high 
impedance transmission lines and the shunt elements are implemented with open-stubs. Figure 1.4 (d) shows a Wilkinson power splitter where the splitter elements are implemented with quarter-wave transmission lines. Figure 1.5 shows the microphotographs of a V-band low-noise amplifier and a $60 \mathrm{GHz}$ band quadrature coupler which were designed using lower-ground transmission lines and implemented in a $90-\mathrm{nm}$ CMOS process as part of the research study of this thesis.

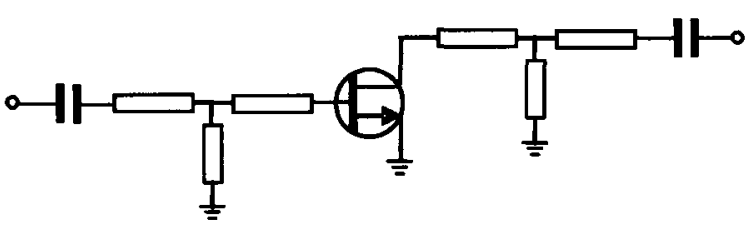

(a)

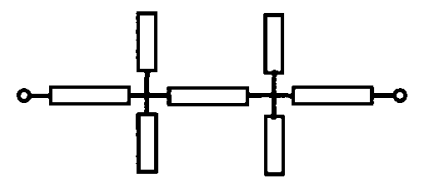

(c)

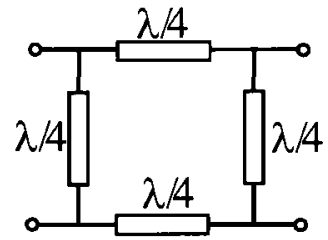

(b)

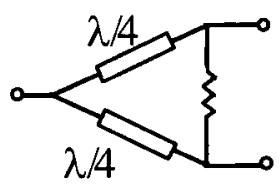

(d)

Figure 1.4: Applications of transmission line elements in mmW building blocks (a) Lownoise amplifier, (b) Quadrature branch-line coupler. (c) Low pass filter, (d) Wilkinson power splitter.

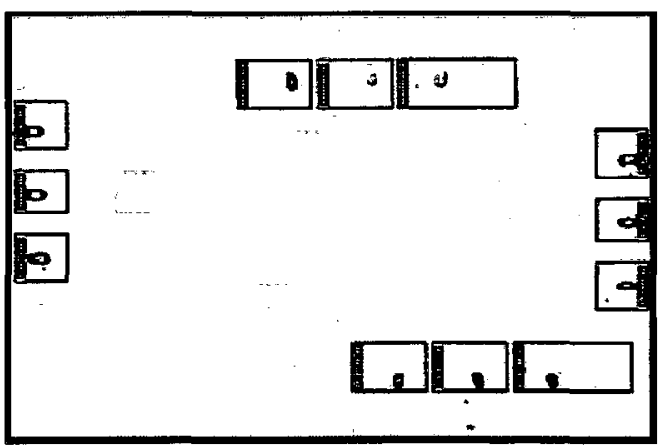

57GHz LNA Chip in 90-nm CMOS

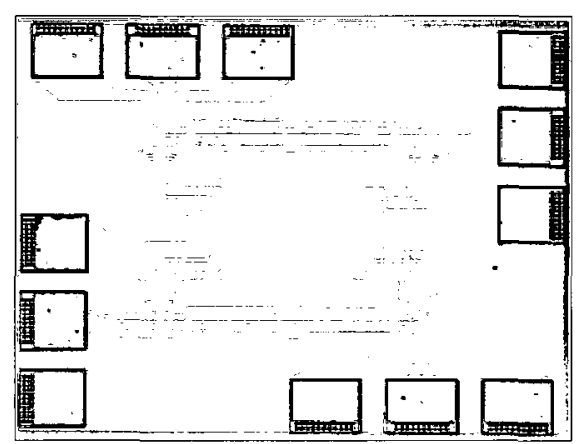

60-GHz 90 Coupler Chip in 90-nm CMOS

Figure 1.5: Microphotograph of $\mathrm{mmW}$ radio front-end building blocks (this work) 


\subsection{CPW Transmission Lines in Silicon Technology}

Coplanar waveguide (CPW) transmission lines [11-15] are commonly used in $\mathrm{mmW}$ systems because of their ease of fabrication, and the elimination of vias to connect shunt elements to the ground plane. The demand for low-cost wireless systems necessitates the use of Si-based CMOS technology because of its low-cost and its potential to develop system-on-chip. However, implementing CPW transmission lines in a CMOS technology presents a serious challenge for achieving a wide range of impedances, Figure 1.6 shows a cross-section view of a Si-based CPW transmission line. This challenge is attributed to the CMOS metal design rules (i.e., maximum/minimum metal width, and minimum spacing between the metal lines). The minimum metal width limits the maximum achievable characteristic impedance; in addition, having a narrow-width line increases the losses of the line and its dependency on the fabrication tolerance.

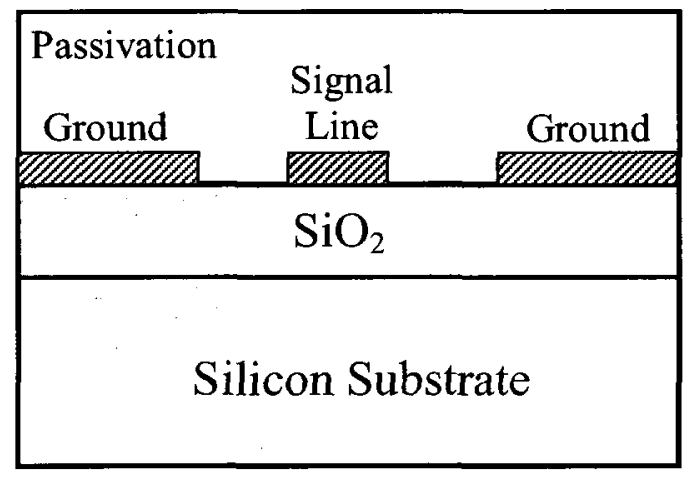

Figure 1.6: Cross-section view of a Si-based CPW transmission line

To increase the characteristic impedance of a CPW line without reducing the width of its signal-line, the gap between the signal-line and its ground strips needs to be increased at 
the expense of increasing the chip-size, the attenuation, and the fabrication cost. On the other hand, the maximum allowable width of the signal-line limits the minimum achievable characteristic impedance. To have a metal width greater than the maximum allowable width, the metal design rules impose having slots in the metal. Having slots in the signal-lines of CPW components in a radio front-end could degrade the system's performance. All these design issue of Si-based transmission lines will be investigated in this thesis. Proposed lower-ground CPW (LG-CPW) transmission lines will be explored to overcome the limitations of the CMOS design rules, and to enhance and reduce the size of passive and active components.

\subsection{Thesis Contributions}

This research has focused on the analysis, design, fabrication, characterization, and applications of LG-CPW transmission lines, to develop V-band CMOS RF front-end building blocks for high data-rate wireless transceivers. The work of this thesis makes the following contributions to increase the competitiveness of CMOS technology to develop V-band low-cost high-speed wireless systems:

- Mitigation of the limitations of the CMOS metal design rules to enable the realization of wide range impedances, which are needed to facilitate the design of $\mathrm{mmW}$ radio front-end components. This work resulted in several publications [21]-[24],[97],[98]. 
- Significant size-reduction in the passive circuit elements by using the proposed LG-CPW transmission lines.

- Design and development of a V-band CMOS low noise amplifier which features low-noise figure, high gain, high linearity, and low power consumption.

- Design and development of a $60 \mathrm{GHz}$ band CMOS quadrature coupler which features very small size in comparison with the state-of-the-art 60 $\mathrm{GHz}$ couplers.

\subsection{Thesis Organization}

This thesis is organized to provide a logical flow through the following chapters:

Chapter 2, Transmission Lines in mmW Radio Front-Ends, provides an overview of the impact of integrated transmission line elements of the radio front-end on the system performance. Analysis of wave propagation in transmission lines based on Maxwell's equations and equivalent circuit model are introduced and discussed. Also an overview of the most common planar transmission lines is provided.

Chapter 3, mmW Silicon-based Lower-Ground CPW Transmission Lines, focuses on the analysis and characterization of different Si-based $\mathrm{mmW}$ multilayer transmission lines including: conventional CPW, vertical ground plane CPW, and LG-CPW. Parameters extraction using chain matrix de-embedding is explained and used to extract 
the measured parameters of transmission line test structures. Experimental measurements and EM simulation results including the characteristic impedance, the attenuation, and the effective dielectric constant of conventional CPW and LG-CPW test structures in a 90-nm CMOS process are presented and discussed.

Chapter 4, Design of a V-band Si-based LNA using LG-CPW Transmission Lines, introduces the design approach of a single-stage LG-CPW low-noise amplifier; characterization of small and large signal performance, electromagnetic simulation, and comparison with the state-of-the-art V-band CMOS LNAs are presented and discussed.

Chapter 5, Design of a Reduced-Size V-band CMOS LG-CPW $90^{\circ}$ Coupler, focuses on the design approach of a compact $60-\mathrm{GHz}$ quadrature coupler in $90-\mathrm{nmCMOS}$ process. Experimental characterization, electromagnetic simulation, and comparison with the state-of-the-art $60 \mathrm{GHz}$ CMOS couplers are presented and discussed.

Chapter 6, Conclusions and Future Work, summarizes the research of this thesis and provides suggestions for future research and potential applications of CMOS LG-CPW transmission lines in mmW high-data rate wireless systems. 


\section{Chapter 2}

\section{Transmission Lines in mmW Radio Front- Ends}

The objective of this chapter is to provide a theoretical foundation for the discussion in the remaining chapters of this thesis. An overview of a wireless transceiver and the impact of the transceiver's transmission line elements on the system's performance is presented. Analysis of wave propagation in transmission lines based on Maxwell's equations and an equivalent circuit model are also discussed, and an overview of the most common planar transmission lines is provided.

\subsection{Radio Transceiver Front-Ends}

Wireless transceivers are examples of radio systems which employ many transmission line circuit-elements. Recently, the increasing demand for high-data rate wireless communications and the continuing improvements in silicon technology, as well as the availability of $7-\mathrm{GHz}$ bandwidth in the unlicensed $60-\mathrm{GHz}$ ISM band have created significant interest in the development of low-cost CMOS mmW wireless transceivers [16]-[19]. Figure 2.1 shows a block diagram of a $\mathrm{mmW}$ radio front-end [20] incorporating Si-based components which were developed as part of this research [21][24]. In Si-based radio front-ends, passive components including filters and couplers are normally implemented off-chip because of their large size, and the losses associated with the silicon substrate [25]-[28]. Meanwhile, having off-chip passive components makes 
the transceiver bulky and expensive. Hence, to meet the objective of low-cost systems, the transceivers of the systems need to feature full integration with reduced-size passive components. The size of the transceiver can also be reduced by using a Zero-IF transceiver architecture [20], which eliminates the need for filters at the outputs of the mixers. However, Zero-IF transceivers have other design challenges such as dc offset which could impact the analog-to-digital converters (ADC) in the transceiver.

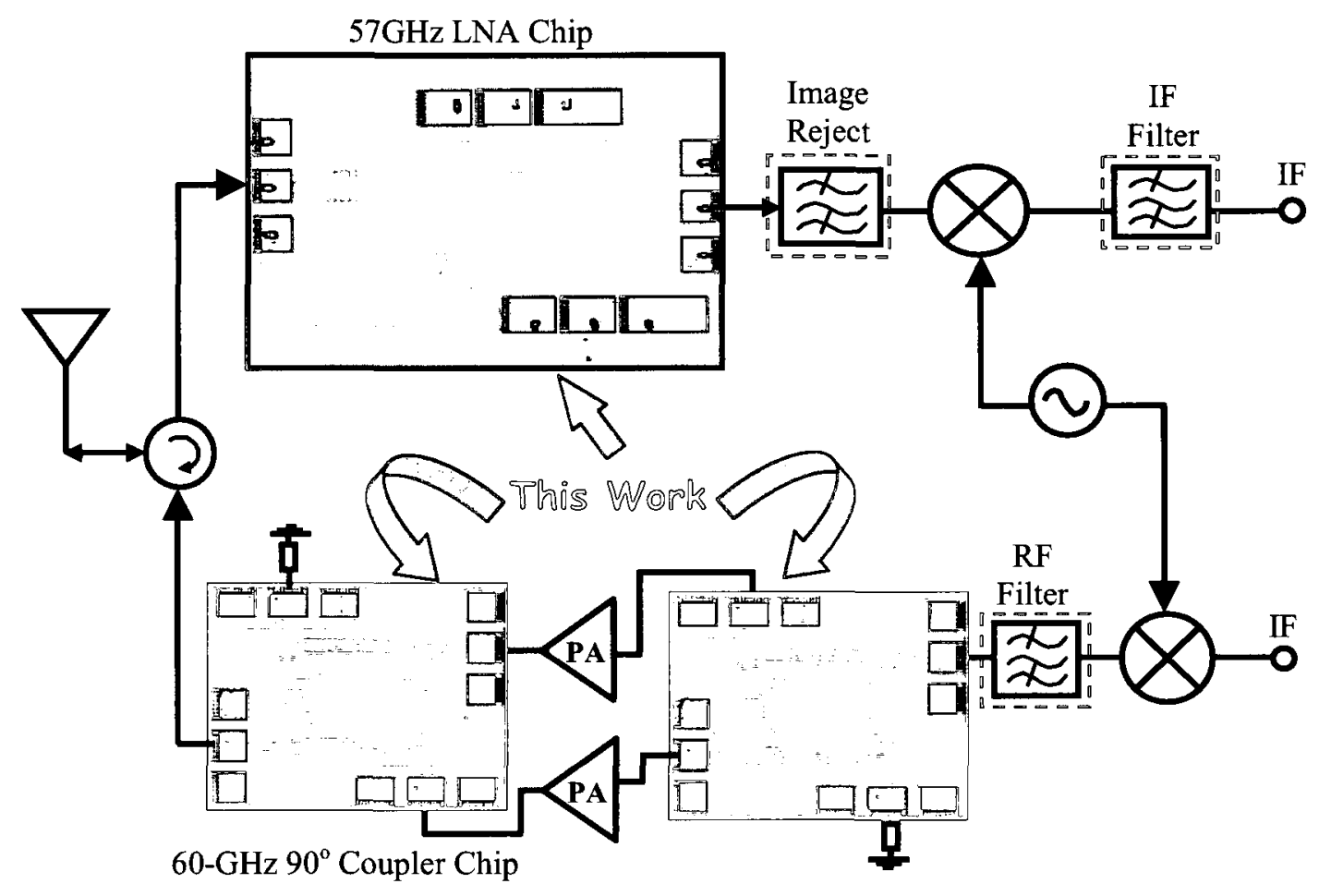

Figure 2.1: Block diagram of mmW Radio Front-End transceiver 
One of the key design parameters which impacts the performance of a wireless system is the receiver's noise figure of the system, $N F$, that is given by

$$
N F=10 \log \left(F_{1}+\sum_{i=2}^{N} \frac{F_{i}-1}{\prod_{j=1}^{i-1} G_{j}}\right)
$$

where $N$ is the number of stages in the receiver, $F_{i}$ is the noise factor of the $i$-th stage, and $G_{j}$ is the gain of the $j$-th stage. Eq. (2.1) indicates that the noise figure of the first component in the receiver chain is the one which dominates the receiver noise figur. Therefore, the passive elements of the receiver front-end such as the transmission lines between the antenna and the input of the low-noise amplifier, and the distributed matching elements of the LNA have significant impact on the receiver performance. These passive elements must have low attenuation, low dispersion, and a wide range of impedances to facilitate the design of the matching networks as well as passive and active components in the radio front-end. The noise figure of the receiver affects the receiver sensitivity which is given by

$$
R x \text { Sens. }=-174 d B m / H z+N F[d B]+10 \log (B W)+S N R
$$

where $N F$ is the receiver's noise figure, $S N R$ is the signal-to-noise ratio, and $B W$ is the channel bandwidth. In the transmitter chain of a radio front-end, the insertion losses of the transmission lines between the antenna and the output of the power amplifier, as well as the losses of the matching elements of the power amplifier influence the level of transmit power which impacts the system's link budge and the communication range. The 
communication range of a wireless link influences the number of transceivers needed to ensure a reliable link. Therefore, the transmission line interconnects and passive elements in the radio front-ends of wireless systems contribute not only to the system performance but also to the cost effectiveness of the system.

\subsection{State-of-the-art mmW Transmission Lines}

There are different types of $\mathrm{mmW}$ transmission lines and each type of these lines has its advantage and disadvantage. The choice of the most suitable type or combinations of them to achieve optimal performance depends on the circuit application. Figure 2.2 shows the most commonly used mmW transmission lines.

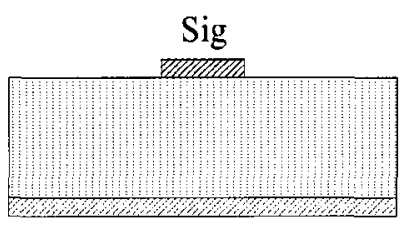

(a)

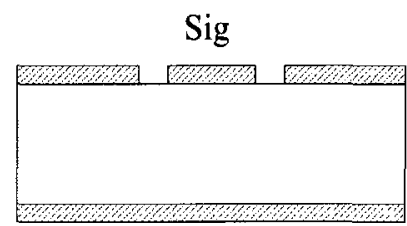

(c)

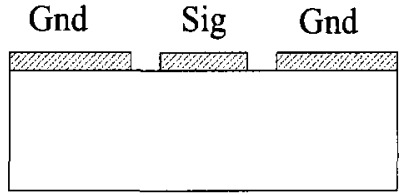

(b)

Dielectric

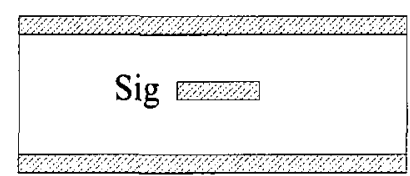

(d)

Figure 2.2: Cross-section of $\mathrm{mmW}$ planar transmission Lines (a) Microstrip, (b) Coplanar waveguide, (c) Conductor-backed CPW, (d) Strip line.

Figures 2.2 (a)-(d) [29]-[33] illustrate the microstrip line, coplanar waveguide (CPW), conductor backed $\mathrm{CPW}$, and strip line, respectively. The fundamental mode of 
propagation of these transmission lines is often referred to as quasi-TEM because of its similarity with pure TEM modes (Transverse-Electro-Magnetic).

Microstrip lines are the most common type of the planar transmission lines used in microwave circuits. Nevertheless, because of the limited impedance range and because of the dispersion phenomena (frequency dependency of the line parameters), the CPW represents a better choice for high frequency operations. In microstrip lines, the height of the substrate is fixed by the fabrication process, therefore, the width of the signalconductor is the only design parameter that can be used to control the line's impedance. Thus, microstrip lines do not provide design flexibility to achieve high impedances.

Meanwhile, CPW transmission lines provide design flexibility since the impedance can be controlled not only by the width of the signal-conductor, but also by the gap between the signal-conductor and the ground-plane strips. In addition, because both of the signal-conductor and the ground-plane strips are placed on the same metal layer, no vias will be required to connect shunt components to the ground plane. Furthermore, better isolation can be obtained with $\mathrm{CPW}$ lines since the ground-plane strips act as a shield. At discontinuities in the signal-line of a CPW, the two ground-plane strips must be kept at the same potential in order to suppress any undesired propagation modes. For this reason, air bridges connecting the two ground-plane strips must be included in the design. Although CPW lines provide design flexibility, they have design challenges when they are implemented on silicon substrates. Some of these challenges are due to the lossy Sisubstrate and the technology design rules which limit the realization of a wide range of 
extreme impedances. Analysis and characterization of Si-based $\mathrm{mmW}$ transmission lines will be discussed and presented in Chapter 3 .

\subsection{Analysis of mmW Transmission Lines}

The analysis of transmission lines in $\mathrm{mmW}$ integrated circuits is essential for determining the key design parameters of the line. Such parameters include the characteristic impedance, the attenuation factor, and the effective dielectric constant. The analysis can be performed based on the wave propagation phenomena which are described by Maxwell's electromagnetic field equations.

\subsubsection{Maxwell's Electromagnetic Field Equations}

Electromagnetic wave propagation in transmission lines can be examined from the solution of Maxwell's field equations [30], which are given by:

$$
\begin{aligned}
& \nabla \times \vec{E}=-\frac{\partial \vec{B}}{\partial t} \\
& \nabla \times \vec{H}=J+\frac{\partial \vec{D}}{\partial t} \\
& \nabla \cdot \vec{D}=\rho_{v} \\
& \nabla \cdot \vec{B}=0
\end{aligned}
$$

and the material equations, which are given by:

$$
\begin{aligned}
& \vec{D}=\varepsilon \vec{E}=\varepsilon_{o} \varepsilon_{r} \vec{E} \\
& B=\mu \vec{H}=\mu_{o} \mu_{r} \vec{H}
\end{aligned}
$$




$$
J=\sigma \vec{E}
$$

where $E$ is the electric field intensity (volt $/ \mathrm{m}), H$ is the magnetic field intensity $(\mathrm{A} / \mathrm{m}), D$ is the electric flux density $\left(\right.$ Coulomb $\left./ \mathrm{m}^{2}\right), B$ is the magnetic flux density $\left(\mathrm{Wb} / \mathrm{m}^{2}\right), J$

$\left(A / m^{2}\right)$ is the current density, and $\rho_{v}\left(C\right.$ Coulomb $\left./ \mathrm{m}^{3}\right)$ is the volume charge density; $\varepsilon_{o}$ and $\varepsilon_{r}$ are the permittivity of the vacuum and the relative permittivity of the medium; $\mu_{o}$ and $\mu_{r}$ are the magnetic permeability of the vacuum and relative permeability of the medium, and $\sigma$ is the electric conductivity of the medium. The field configurations (i.e., field distributions) in a transmission line are called propagation modes and every propagation mode has its own propagation characteristics (i.e., attenuation/unit length and phase velocity). The fundamental mode of propagation in a transmission line is a quasi-TEM, where both the electric and magnetic field components are transverse to the direction of propagation. The wave propagation in $\mathrm{mmW}$ transmission lines can also be described by the use of Helmholtz equation which is discussed in the next sub-section.

\subsubsection{Helmholtz Equation}

In order to have a generalized Helmholtz equation which describes the electric and magnetic fields in transmission lines, the medium is considered source free $(\rho=J=0)$ and the fields are considered to be time harmonic (i.e., fields vary at sinusoidal frequency $\omega$ ). With these assumptions, Maxwell's equations can be written as:

$$
\begin{aligned}
& \nabla \times \vec{E}=-j \omega \mu \vec{H} \\
& \nabla \times \vec{H}=j \omega \varepsilon \vec{E}
\end{aligned}
$$




$$
\begin{aligned}
& \nabla \cdot \varepsilon \vec{E}=0 \\
& \nabla \cdot \mu \vec{H}=0
\end{aligned}
$$

Taking the divergence of (2.10) and (2.11) yields:

$$
\begin{aligned}
& \nabla \cdot(\nabla \times \vec{E})=-j \omega \nabla \cdot \mu H \\
& \nabla \cdot(\nabla \times \vec{H})=j \omega \nabla \cdot \varepsilon E
\end{aligned}
$$

In order to derive the Helmholtz equation for the electric field vector $E$, one can multiply (2.10) by $\mu^{-I}$ and then take the curl of the expression to have:

$$
\nabla \times\left(\mu^{-1} \nabla \times \vec{E}\right)=-j \omega \nabla \times H
$$

Substituting (2.11) in (2.16) yields:

$$
\nabla \times\left(\mu^{-1} \nabla \times \vec{E}\right)=\omega^{2} \varepsilon E
$$

Equation (2.17) is the generalized Helmholtz equation for the electric field. The generalized Helmholtz equation for the magnetic field can be obtained by the same approach, which gives:

$$
\nabla \times\left(\varepsilon^{-1} \nabla \times \vec{H}\right)=\omega^{2} \mu H
$$

Equations (2.17) and (2.18) can be further simplified by assuming the permittivity $\varepsilon$ and the permeability $\mu$ of the medium are constant. Using the vector identity:

$$
\nabla \times(\nabla \times \vec{E})=\nabla(\nabla \cdot E)-\nabla^{2} E
$$

the generalized Helmholtz equations can be written as: 


$$
\begin{aligned}
\nabla^{2} E+\omega^{2} \varepsilon \mu E & =0 \\
\nabla^{2} H+\omega^{2} \varepsilon \mu H & =0
\end{aligned}
$$

Equations (2.20) and (2.21) are also known as the wave equations.

\subsubsection{Telegrapher's Equations}

Transmission lines can be analyzed by the solution of Maxwell's equation and by using the distributed-circuit approach. The solution of Maxwell's equations involves three space variables and the time variable. However, the distributed-circuit approach involves only one space variable in addition to the time variable. In this sub-section, the circuit approach will be used to analyze the transmission line in terms of voltage, current, and impedance. Figure 2.3 shows an equivalent circuit model for a transmission line section of a length $d z$.

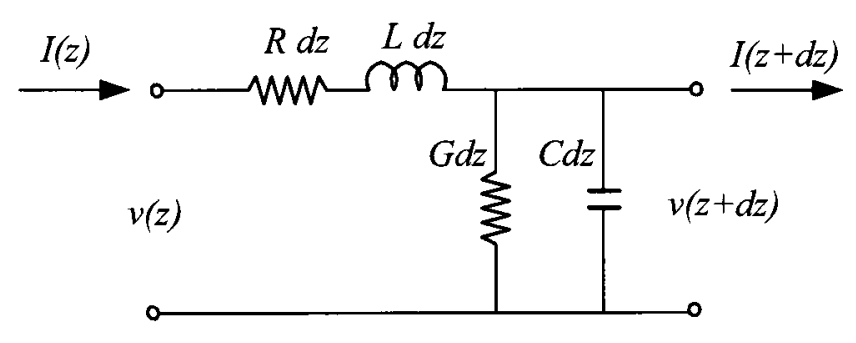

Figure 2.3: Circuit model of a transmission line section of a length $\mathrm{dz}$.

The parameters of the section $d z$ are resistance per unit length $R$, inductance per unit length $L$, conductance per unit length $G$, and capacitance per unit length $C$. 
Applying both Kirchhoff's voltage and current laws to the circuit shown in Figure 2.3 results in a system of first-order coupled differential equations, which are referred to as the telegrapher's equations of a transmission line and are given by:

$$
\begin{gathered}
\frac{\partial V}{\partial z}=-\left(R I+L \frac{\partial I}{\partial t}\right) \\
\frac{\partial I}{\partial z}=-\left(G V+C \frac{\partial V}{\partial t}\right)
\end{gathered}
$$

With some algebraic manipulation, one can obtain the following second-order differential equations for the transmission line section:

$$
\begin{aligned}
& \frac{\partial^{2} V}{\partial z^{2}}=R G V+(R C+G L) \frac{\partial V}{\partial t}+L C \frac{\partial^{2} V}{\partial t^{2}} \\
& \frac{\partial^{2} I}{\partial z^{2}}=R G I+(R C+G L) \frac{\partial I}{\partial t}+L C \frac{\partial^{2} I}{\partial t^{2}}
\end{aligned}
$$

Eq. (2.24) can be written in a general form as:

$$
\frac{\partial^{2} \psi}{\partial z^{2}}=R G \psi+(R C+G L) \frac{\partial \psi}{\partial t}+L C \frac{\partial^{2} \psi}{\partial t^{2}}
$$

where $\psi$ represents either $V$ or $I$ or any other field parameter. Eq. (2.24) can be written in a phasor form as

$$
\frac{\partial^{2} V}{\partial z^{2}}=R G V+(R C+G L) j \omega V-L C \omega^{2} V
$$

Eq. (2.26) can be written as:

$$
\frac{\partial^{2} V}{\partial z^{2}}=(R+j \omega L)(G+j \omega C) V
$$


which can be expressed as:

$$
\frac{\partial^{2} V}{\partial z^{2}}-\gamma^{2} V=0
$$

where:

$$
\gamma=\sqrt{(R+j \omega L)(G+j \omega C)}
$$

The solution of (2.28) is given by:

$$
V(z)=V_{1} e^{-\gamma z}+V_{2} e^{\gamma z}
$$

The first term of (2.30) represents a wave propagating in the positive $z$-direction, while the second term represents a wave propagating in the negative z-direction. The propagation constant $\gamma$, is a complex parameter which consists of the attenuation factor $\alpha$ and the phase constant $\beta$, and is given by:

$$
\gamma=\alpha+j \beta
$$

At millimetre-wave frequencies, $\mathrm{R} \ll \omega L$ and $G \ll \omega C$. By using a binomial expansion, the propagation constant $\gamma$ can be expressed as:

$$
\begin{aligned}
& \gamma=j \omega \sqrt{L C} \sqrt{\left(1+\frac{R}{j \omega L}\right)\left(1+\frac{G}{j \omega C}\right)} \\
& \gamma \approx j \omega \sqrt{L C}\left[\left(1+\frac{1}{2} \frac{R}{j \omega L}\right)\left(1+\frac{1}{2} \frac{G}{j \omega C}\right)\right] \\
& \gamma=\frac{1}{2}\left(R \sqrt{\frac{C}{L}}+G \sqrt{\frac{L}{C}}\right)+j \omega \sqrt{L C}
\end{aligned}
$$

Therefore, the attenuation and phase constant can be written as: 


$$
\begin{aligned}
& \alpha=\frac{1}{2}\left(R \sqrt{\frac{C}{L}}+G \sqrt{\frac{L}{C}}\right) \\
& \beta=\omega \sqrt{L C}
\end{aligned}
$$

Also, the characteristic impedance $Z_{0}$ can be written as:

$$
Z_{o}=\sqrt{\frac{R+j \omega L}{G+j \omega C}}
$$

Since, at millimetre-wave frequencies, $R \ll j \omega L$ and $G \ll j \omega C$, Eq.(2.36) can be written as

$$
Z_{o}=\sqrt{\frac{L}{C}}
$$

From (2.35), the phase velocity $v_{p}$ can be estimated as:

$$
v_{p}=\frac{\omega}{\beta}=\frac{1}{\sqrt{L C}}
$$

The parameters $Z_{o}, \alpha$, and $\beta$ are the key design parameters of millimeter-wave transmission lines. These parameters will be measured and analyzed for Si-based transmission lines in Chapter 3.

\section{i) Medium Loss}

The dielectric material of the transmission lines is normally non perfect and results in a dielectric loss. Since the conductivity of the medium is not perfect (i.e., $\sigma \neq \infty),(2.11)$ can be written as: 


$$
\nabla \times \vec{H}=j \omega \varepsilon\left(1-j \frac{\sigma}{\omega \varepsilon}\right) E=j \omega \varepsilon(1-j \tan \delta) E
$$

where $\tan \delta$ is referred to as the loss tangent of the medium and describes the medium's loss.

\section{ii) Attenuation}

The electrical signals that propagate in transmission lines experience some attenuation due to lossy substrate and imperfect conductors (i.e., $\sigma \neq \infty$ ). At millimeter-wave frequencies, the skin-effect $\delta$ must be taken into account when metal related loss is considered. At these high frequencies, the current density flowing through the metal is concentrated in a metal thickness which is $3 \delta$, where $\delta$ is given by:

$$
\delta=\frac{1}{\sqrt{\pi f \mu \sigma}}
$$

The skin depth $\delta$ is defined as the distance from the medium surface, to where the magnitude of the field of a wave traveling in the medium is reduced to $1 / \mathrm{e}$ (i.e, $37 \%$, $\mathrm{e}$ $=2.718$ ) relative to the wave's magnitude at the medium's surface. The conductor loss can also be described in terms of its surface resistance $R_{s}$ which is given by:

$$
R_{s}=\frac{1}{\sigma \delta}
$$

using (2.40), the surface resistance can be expressed as:

$$
R_{s}=\sqrt{\frac{\pi f \mu}{\sigma}}
$$


The attenuation factor for different Si-based transmission lines will be measured and analyzed in Chapter 3 .

\subsection{Transmission Line Matching Elements}

In CMOS design particularly at higher frequencies, it is important to take into consideration the effect of the metal fill and the design rules of the CMOS process to ensure predictable performance. The metal fill can not be incorporated into the models of the lumped components of the commercial CMOS design kits. Meanwhile, the metal fill can be incorporated with distributed passive elements and simulated using an EM simulator. This makes it desirable to model circuits with distributed components rather than as lumped components. Also, the models of most of the commercial CMOS design kits are validated up to $20 \mathrm{GHz}$ only. Capacitive and inductive transmission lines matching elements can be implemented with open and short transmission line stubs. This can be explained by considering a loaded transmission line as shown in Figure 2.4

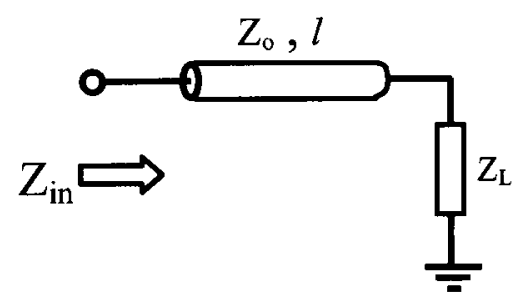

Figure 2.4: Loaded transmission line

Assuming a lossless transmission line, the input impedance $Z_{\text {in }}$ looking into the line can be written as [33] 


$$
Z_{\text {in }}=Z_{o} \frac{Z_{L}+j Z_{o} \tan (\beta l)}{Z_{o}+j Z_{L} \tan (\beta l)}
$$

where $Z_{\mathrm{o}}$ is the line's characteristic impedance, $Z_{\mathrm{L}}$ is the load impedance, and $l$ is the length of the transmission line. If the load is a short or open circuit, the input impedance becomes pure imaginary (no real part exists), such a line is called a stub. With $Z_{\mathrm{L}}=0$ and the line's length less than a quarter-wave length at the operating frequency, the input impedance of the line becomes inductive and (2.43) can be written as

$$
Z_{\text {in }}=Z_{o} \frac{j Z_{o} \tan (\beta l)}{Z_{o}}=j Z_{o} \tan (\beta l)
$$

Thus, with an inductive load, (2.44) can be written as

$$
Z_{\text {in }}=j \omega L=j Z_{o} \tan (\beta l)
$$

where $L$ is the inductance and $\omega$ is the angular frequency. From (2.45) the length of the stub can be calculated as

$$
l_{\text {stub }}=\frac{1}{\beta} \tan ^{-1}\left(\frac{2 \pi f . L}{Z_{o}}\right)
$$

and

$$
\beta=\frac{2 \pi}{\lambda_{g}}, \quad \lambda_{g}=\frac{c}{f \sqrt{\varepsilon_{e f f}}}
$$

where $\lambda_{\mathrm{g}}$ is the wavelength in the medium, $c$ is the speed of light in vacuum, and $\varepsilon_{\text {eff }}$ is the effective dielectric constant. Expressing $L$ in $\mathrm{nH}$ and the frequency in $\mathrm{GHz},(2.46)$ can be written as 


$$
l_{\text {stub }}=\frac{0.3}{2 \pi f \sqrt{\varepsilon_{\text {eff }}}} \tan ^{-1}\left(\frac{2 \pi f . L}{Z_{o}}\right)
$$

From (2.47), to make the length of the stub small, the characteristic impedance needs to be high. In CPW transmission lines, increasing the line's characteristic impedance means either reducing the width of the signal-conductor or increasing the gap (i.e., the space between the signal-line and the ground-plane strips). Reducing the signal line width increases the conductor losses, also, it poses current limitation if the line is used as an RF choke. Furthermore, increasing the gap increases the attenuation and the dispersion.

The length of a capacitive stub can be determined by using the same approach of the inductive stub, except the load of the line should be an open circuit. Thus, the length of the capacitive stub can be written as

$$
l_{s t u b}=\frac{0.3}{2 \pi f \sqrt{\varepsilon_{e f f}}} \tan ^{-1}\left(\frac{2 \pi f Z_{o} \cdot C}{1000}\right)
$$

In (2.48), $C$ is in $\mathrm{pF}$ and $f$ is in GHz. Equations (2.47) and (2.48) can be used to design stubs of high and low impedances to facilitate the design of the matching networks in mmW integrated circuits. However, achieving extreme impedances with CPW or microstrip transmission lines in CMOS technology is limited by the CMOS design rules, Chapter 3 will discuss this issue in details. 


\subsection{Chapter Summary}

In this chapter, the impact of transmission lines and passive circuit-elements of radio front-ends on the system's receiver sensitivity and noise figure was addressed; a theoretical analysis of the propagation characteristics of transmission lines was introduced in order to provide foundations for the discussion in the remaining chapters. A brief overview of commonly used $\mathrm{mmW}$ transmission lines was also presented. 


\section{Chapter 3}

\section{Millimeter-wave Si-based Lower-Ground CPW Transmission Lines}

This chapter focuses on electromagnetic simulation, experimental characterization, and analysis of mmW Si-based lower-ground CPW (LG-CPW) transmission lines and their comparison with conventional CPW. The objective of this chapter is to exploit different LG-CPW transmission lines to achieve an impedance range wider than that of conventional CPW transmission lines and to have low attenuation. Also, an equally important objective is to overcome the geometry limitation imposed by the technology design rules of a CMOS process. Key design parameters including the characteristic impedance, the attenuation factor, and the effective permittivity of LGCPW transmission lines are calculated and compared with those of conventional CPW. Test structures for CPW and LG-CPW transmission lines were fabricated in a 90nm 9-metal layers CMOS technology and characterized to validate the EM simulation results.

\subsection{Transmission Lines in Silicon Technology}

There is a significant interest in implementing transmission line elements and passive components in CMOS technology [34]-[43], to have a true system-on-chip which accommodates both the RF and digital circuitry. However, the realization of transmission lines and passive elements in CMOS at $\mathrm{mmW}$ frequencies presents 
design challenges. One of these challenges is due to the low resistivity Si-substrate which causes a significant loss. Another challenge is due to the geometry limitations imposed by the technology design rules, which limit the realization of a wide range of impedances that might be required for filter design and matching networks. To overcome the problem of the lossy Si-substrate, various solutions including the use of low-loss dielectric, high resistivity substrates, and the removal of the silicon under the signal line to reduce the losses of the lines have been investigated. However, these solutions increase the cost and complexity of fabrication. In this study, we propose the utilization of the multiple metal layers that are available in CMOS technology to design on-chip transmission lines which could provide a wide range of impedances and low loss without violating the CMOS design rules. Figure 3.1 shows a crosssection of the metal layers in a typical CMOS process with 9-metal layers.

\begin{tabular}{|c|c|c|c|}
\hline \multicolumn{4}{|c|}{ Top passivation } \\
\hline$\square \mathrm{M9}$ & \multirow[b]{4}{*}{$\mathrm{SiO}_{2}$} & \multirow{2}{*}{ M8 } & \\
\hline$\exists_{\mathrm{M} 7}$ & & & 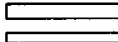 \\
\hline & & M6 & \\
\hline & & M4 & \\
\hline M1 & & M2 & \\
\hline & $\begin{array}{l}\text { Sul } \\
s=\end{array}$ & $\begin{array}{l}\text { rate } \\
\text { S/m }\end{array}$ & \\
\hline
\end{tabular}

Figure 3.1: Cross-section view of the metal layers in a typical RF-CMOS process with 9-metal layers

In silicon technology, transmission lines such as microstrip [25]-[26], [44] are implemented by utilizing the top metal layer of the inter-metal stack to realize the signal-line and any of the lower metal layers to realize the ground plane. In this type 
of transmission lines, the ground plane acts as an electric wall that prevents the electric field lines from penetrating into the silicon substrate, thus, eliminating the contribution of the losses associated with the silicon substrate. Figure 3.2 shows a cross-section view of a CMOS microstrip line and its electric field distribution. In a CMOS microstrip, although the ground plane prevents the electric field lines from reaching the silicon, it results in a reduced distance between the signal-line and the ground plane of the structure; which limits the maximum characteristic impedance that can be achieved. In spite of the commonly use of microstrip lines and their ease of fabrication, they have drawbacks which include: poor electromagnetic (EM) isolation between adjacent lines, and the need for vias to connect shunt components to the ground planes.

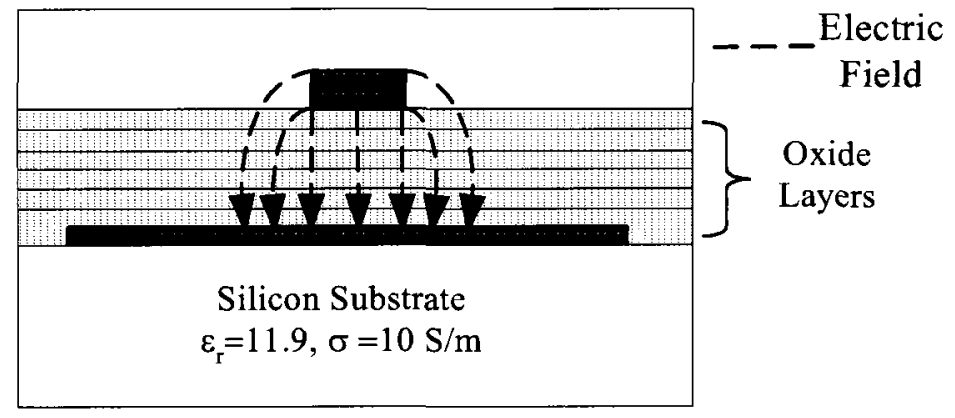

Figure 3.2: Cross-section view of a Si-based microstrip line and its electric field distribution

In microstrip transmission lines, the impedance of the line is a function of the line's width and the $\mathrm{SiO}_{2}$ thickness but the $\mathrm{SiO}_{2}$ thickness is fixed by the technology process. Therefore, the range of the impedances that can be obtained by a microstrip 
line depends mainly on one parameter which is the line's width. This limits the design flexibility of microstrip compared to CPW structures.

\subsection{Silicon-based CPW Transmission Lines}

CPW transmission lines [14]-[15], [45] are implemented in Si-based CMOS technology by placing the signal-conductor and its two ground-plane strips on the top metal layer of the inter-metal stack as shown in Figure 3.3. Figure 3.3 shows a 3-D view ( Si-substrate is not shown for clarity) of a CMOS CPW structure and its field distribution. Having the three metal strips on the same metal layer facilitates the grounding of shunt components. In addition, the three design parameters which include the width of the signal-line, the width of the ground-strips, and the gap between the signal-conductor and the ground-strips, enable achieving an impedance range wider than that of microstrip.
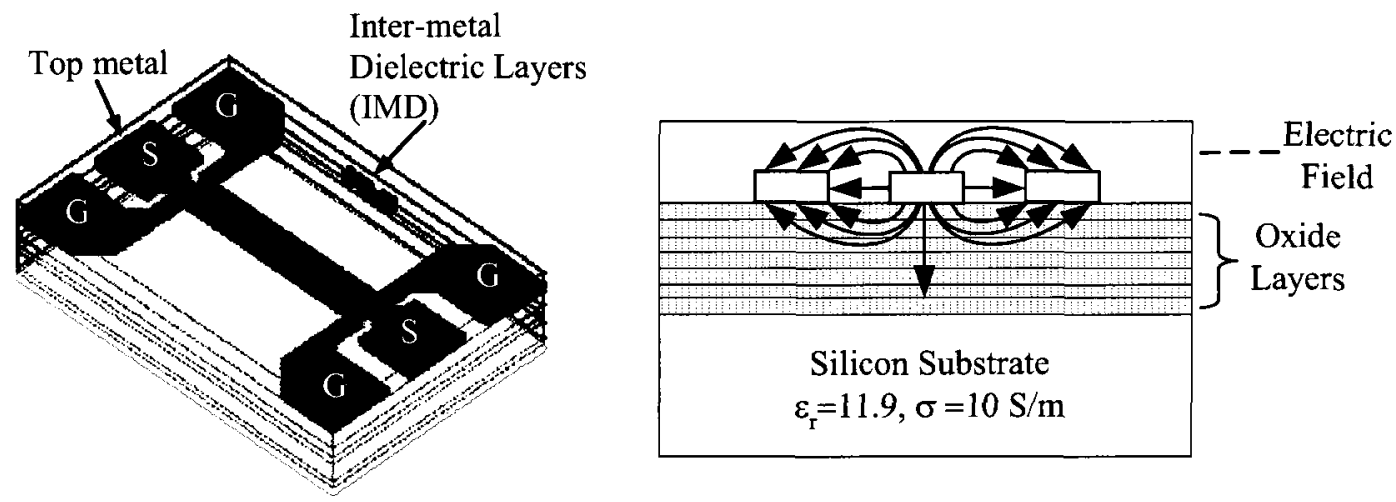

Figure 3.3: CMOS CPW Structure (a) 3-D view (Si-substrate is not shown), (b) Electric field distribution 
Furthermore, the ground strips of CPW lines improve the isolation between adjacent CPW lines [45]. Good isolation between the transmission lines in an RF front-end is essential to prevent the possibility of having unstable amplifiers or cross-talk. In CPW structures, at any discontinuity in the line the two ground-plane strips of the structure must have the same potential (ground equalization), to prevent the propagation of undesired modes that increase losses over the structure. For Si-based CPW transmission lines, ground equalization can be achieved by using a metal underpass which connects the two ground-plane strips. Although CPWs provide an impedance range wider than that of microstrips, the impedance range of CPWs is still limited by the design rules of the CMOS technology process.

\subsection{Si-based Lower-Ground CPW Transmission Lines}

In conventional Si-based CPW lines, the achievable impedances are in the range of about 35-80 ohms, and other impedances outside this range can not be fabricated reliably. Meanwhile, some applications such as reduced-size couplers, power combiners/splitters, phase shifters, and filters require high-impedance lines to achieve size-reduction by capacitive loading. Increasing the impedance of a CPW to values higher than $80 \mathrm{ohms} \mathrm{leads} \mathrm{to} \mathrm{a} \mathrm{significant} \mathrm{increase} \mathrm{in} \mathrm{the} \mathrm{attenuation} \mathrm{due} \mathrm{to} \mathrm{the}$ increase in the ohmic loss. Furthermore, reducing the line-width to achieve high impedance makes the transmission line sensitive to the fabrication tolerance, which might severely impact the electrical performance of the transmission line. Achieving high impedance in silicon technology was reported and obtained by the use of an elevated centre-conductor [46]-[54] of the CPW, this was achieved either by using 
metallic posts or by removing the Si-dioxide under the signal-line. However, these solutions increase the fabrication cost. In this research, we explore the utilization of the available multiple metal layers in CMOS technology to achieve an impedance range wider than that of the conventional CPW and particularly higher impedances. Moving the ground-plane strips of a CPW structure to a metal layer lower than that of the signal-conductor, decreases the capacitance/unit length of the line which increases the line's characteristic impedance. Since the lower metal layers have thickness smaller than that of the top metal layer, the thickness of the lowered ground-plane strips can be increased by combing different metal layers through vias to reduce the attenuation. Figure 3.4 shows a 3-D view and the field distribution of the proposed lower-ground CPW (LG-CPW) transmission line structure.

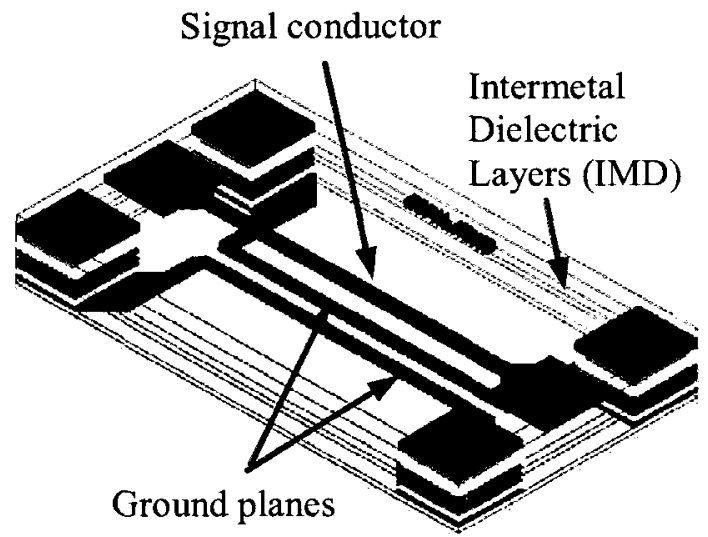

(a)

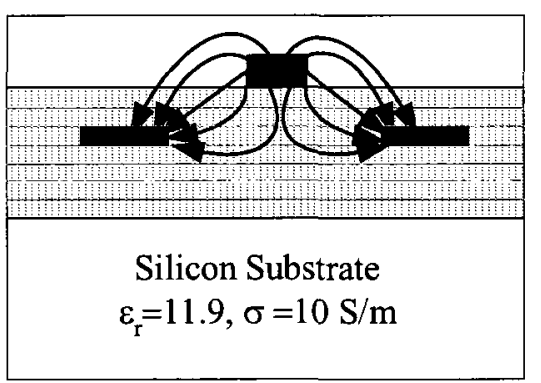

(b)

Figure 3.4: CMOS LG-CPW transmission line structure (a) 3D view, (b) Cross section

In LG-CPW structures, the ground-plane strips can overlap with the signal-line because they are not on the same metal layer; thus, lowering the impedance of the line 
without increasing the metal-width. Keeping the metal width within the limits of the process design rules eliminates need for introducing slots in the metal as required by the CMOS design rules. To reduce the attenuation of a LG-CPW structure, the thickens of the ground strips can be increased by forming a vertical ground which combines the lower metal layers through vias. Figure 3.5 shows a 3-D view of a vertical lower ground CPW (VLG-CPW) transmission line structure.
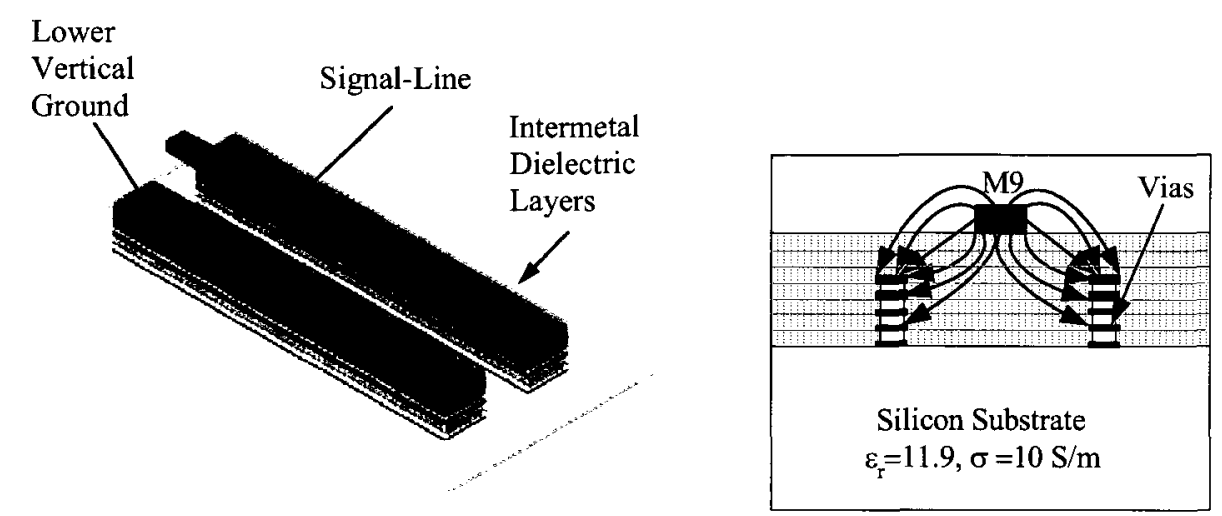

Figure 3.5: 3-D view and a cross section of a vertical LG-CPW transmission line

Thus, LG-CPW transmission line structures provide design flexibility to achieve a wide range of impedances and lower attenuation, as well as mitigating the geometry limitations imposed by the CMOS design rules. 


\subsection{Analysis of Si-based LG-CPW Transmission Lines}

In this analysis, a lumped-element equivalent circuit model [55] as shown in Figure 3.6 is used to analyze a Si-based LG-CPW transmission line. In Figure 3.6, $R$ represents the resistive loss and the conductor skin effect resistance per unit length; and $\mathrm{L}$ represents the skin effect and the self-inductances per unit length of the transmission line, respectively; $C_{\mathrm{ox}}$ is the shunt capacitance per unit length of the silicon dioxide, $C_{\text {sub }}$ is the shunt capacitance per unit length of the Si-substrate, and $G_{\text {sub }}$ is the shunt conductance per unit length of the Si-substrate. Figure 3.6 (b) is an equivalent model of Figure 3.6 (a).

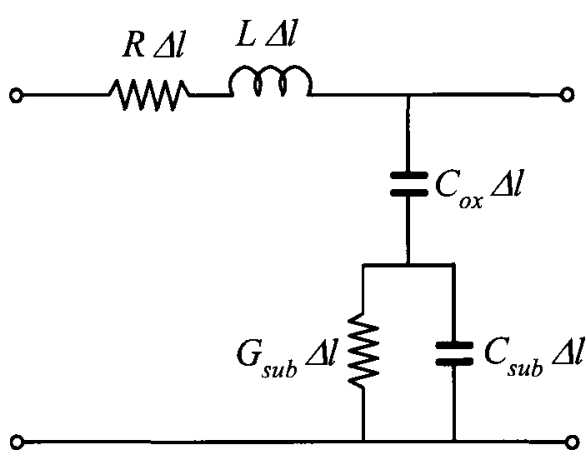

(a)

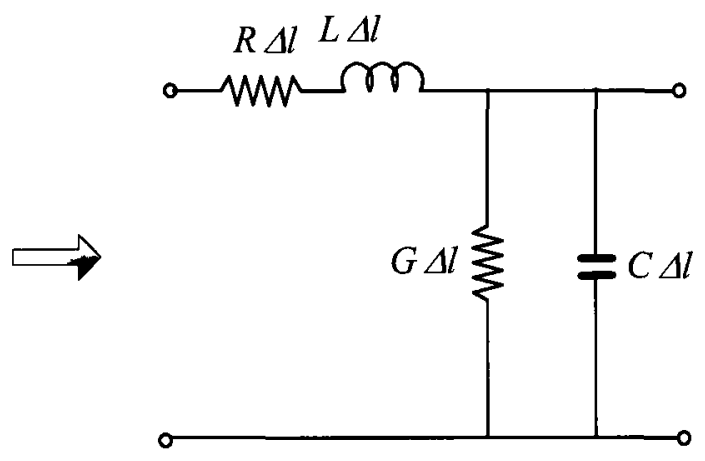

(b)

Figure 3.6: Equivalent circuit model of a CMOS LG-CPW transmission line section $\Delta l$ (a) Detailed lumped-element model (b) Simplified equivalent model

In Figure 3.6-b, $G$ is the equivalent shunt conductance per unit length, and $C$ is the equivalent capacitance per unit length. Both $G$ and $C$ can be expressed in terms of $C_{\mathrm{ox}}, C_{\text {sub }}$, and $G_{\text {sub }}$ as follows 


$$
\begin{aligned}
& C=C_{o x} \cdot \frac{1+C_{s u b}\left(C_{o x}+C_{s u b}\right)\left(\frac{\omega}{G_{s u b}}\right)^{2}}{1+\left(C_{o x}+C_{s u b}\right)^{2}\left(\frac{\omega}{G_{s u b}}\right)^{2}} \\
& G=\frac{\omega^{2} \cdot C^{2}{ }_{o x} \cdot G_{s u b}}{G_{s u b}^{2}+\omega^{2}\left(C_{o x}+C_{s u b}\right)^{2}}
\end{aligned}
$$

From (2.29), knowing the propagation constant $\gamma$, the transmission line parameters can be can be determined and the characteristic impedance $Z_{\mathrm{o}}$ can be estimated using (2.36). The propagation constant can be obtained from the chain matrix $(A B C D)$ of the transmission line, which can be obtained from the line's scattering parameters (Sparameters) by using following transformation:

$$
\left[\begin{array}{ll}
A & B \\
C & D
\end{array}\right]=\left[\begin{array}{ll}
\frac{\left(1+S_{11}\right)\left(1-S_{22}\right)+S_{12} S_{21}}{2 S_{21}} & Z_{o} \frac{\left(1+S_{11}\right)\left(1+S_{22}\right)-S_{12} S_{21}}{2 S_{21}} \\
\frac{1}{Z_{o} \frac{\left(1-S_{11}\right)\left(1-S_{22}\right)-S_{12} S_{21}}{2 S_{21}}} & \frac{\left(1-S_{11}\right)\left(1+S_{22}\right)+S_{12} S_{21}}{2 S_{21}}
\end{array}\right]
$$

The $(A B C D)$ matrix can also be represented in terms of the propagation constant by using the following transformation:

$$
\left[\begin{array}{ll}
A & B \\
C & D
\end{array}\right]=\left[\begin{array}{cc}
\cosh (\gamma l) & z_{o} \sinh (\gamma l) \\
z_{o}^{-1} \sinh (\gamma l) & \cosh (\gamma l)
\end{array}\right]
$$

where $l$ is the transmission line length, and $\gamma$ is the propagation constant. From (3.4) and (2.3), $\gamma$ is given by: 


$$
\gamma=\frac{\cosh ^{-1}(A)}{l}=\alpha+j \beta
$$

where $\alpha$ is the attenuation factor $(\mathrm{Np} /$ length) and $\beta$ is the phase constant ( $\mathrm{rad} /$ length). Knowing the $A B C D$ parameters, the characteristic impedance and the attenuation of the transmission line can be calculated as

$$
\begin{aligned}
Z_{o} & =\sqrt{\frac{B}{C}} \\
\alpha & =\operatorname{Re}\left[\frac{\cosh ^{-1}(A)}{l}\right]
\end{aligned}
$$

from (3.5) the phase constant can be estimated as

$$
\beta=\operatorname{Im}\left[\frac{\cosh ^{-1}(A)}{l}\right]
$$

Knowing the phase constant, the $\varepsilon_{e f f}$ and the quality factor, Q, can be calculated as

$$
\begin{aligned}
\varepsilon_{\text {eff }} & =\left(\frac{0.3 \beta}{2 \pi \cdot f}\right)^{2} \\
Q & =\frac{\beta}{2 \alpha}
\end{aligned}
$$

Having the characteristic impedance $Z_{o}$ and the propagation constant $\gamma$ of the transmission line, the frequency-dependent equivalent circuit parameters per unit length $R, L, G$, and $C$ of the transmission line can be calculated using (2.29) and (2.36) which yield: 


$$
\begin{array}{ll}
R=\operatorname{Re}\left[\gamma \cdot Z_{o}\right], & L=\operatorname{Im}\left[\frac{\gamma \cdot Z_{o}}{\omega}\right] \\
G=\operatorname{Re}\left[\frac{\gamma}{Z_{o}}\right], & C=\operatorname{Im}\left[\frac{\gamma}{Z_{o} \cdot \omega}\right]
\end{array}
$$

In this study, the characteristic impedance, attenuation factor, and effective permittivity of CMOS conventional CPW and LG-CPW are calculated based on an electromagnetic (EM) simulation. The simulation results are validated by experimental measurements. 


\subsection{Extraction of Measured Parameters using Chain Matrix De-embedding}

In this section, the characterization [56]-[57] of the transmission lines is carried out by using chain-matrix de-embedding technique [58] to extract the line's parameters. This technique is based on the use of the scattering parameters of two identical test structures of different lengths, to obtain the chain-matrix $(A B C D)$ of an embedded transmission line section. Figure 3.7 shows two identical CPW test structures of different lengths for determining the $A B C D$ matrix of an embedded CPW line.

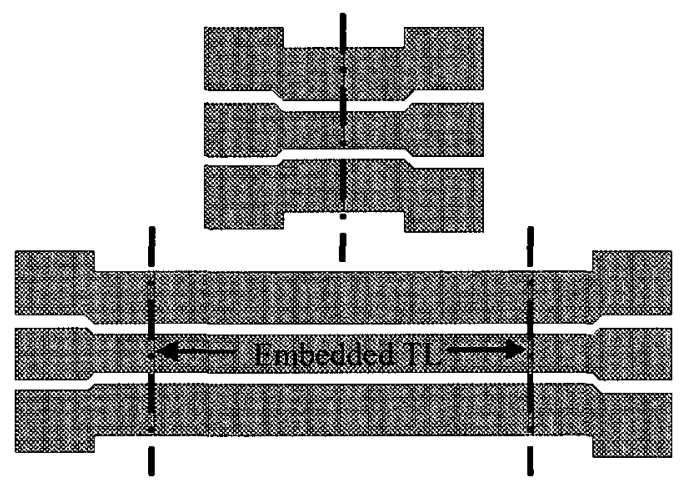

Figure 3.7: Two identical CPW test structures of different lengths for extracting the $A B C D$ parameters of an embedded CPW transmission line.

In Figure 3.7, the long test structure can be represented as a transmission line (TL) section embedded between two test pads $\left(P_{1 \mathrm{~L}}\right.$ and $\left.P_{2 \mathrm{~L}}\right)$ as shown in Figure 3.8. Similarly, the short test structure can be represented as two test pads ( $P_{1 \mathrm{~S}}$ and $\left.P_{2 \mathrm{~S}}\right)$ connected back-to-back and have the same size as the test pads of the long structure. Thus, five chain matrices are involved in this de-embedding process. 


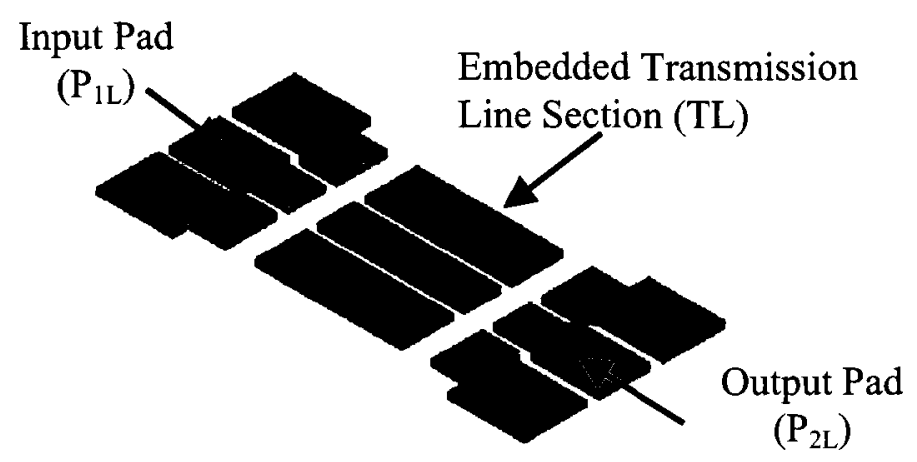

Figure 3.8: Representation of the long CPW test structure

The chain matrix of the long structure $\left(\left[A_{L}\right]\right)$ represents a multiplication of the chain matrices of the test pads $\left(\left[A_{P I L}\right]\right.$ and $\left.\left[A_{P 2 L}\right]\right)$ and the embedded line $\left(\left[A_{T L}\right]\right)$, thus,

$$
\left[A_{L}\right] \equiv\left[A_{P 1 L}\right] \cdot\left[A_{T L}\right] \cdot\left[A_{P 2 L}\right]
$$

where

$$
\left[A_{T L}\right]=\left[\begin{array}{cc}
\cosh (\gamma l) & z_{o} \sinh (\gamma l) \\
z_{o}^{-1} \sinh (\gamma l) & \cosh (\gamma l)
\end{array}\right]
$$

$l$ is the length of the embedded $\mathrm{TL}$ and $Z_{o}$ is the characteristic impedance of the transmission line. The chain matrix of the short structure $\left(\left[A_{\mathrm{S}}\right]\right)$ is the product of the chain matrices of the test pads $\left(\left[A_{P I S}\right]\right.$ and $\left.\left[A_{P 2 S}\right]\right)$. The chain matrices of the short and long test structures can be obtained from their measured scattering parameters by using the following equation:

$$
\left[\begin{array}{ll}
a_{11} & a_{12} \\
a_{21} & a_{22}
\end{array}\right]=\left[\begin{array}{ll}
\frac{\left(1+S_{11}\right)\left(1-S_{22}\right)+S_{12} S_{21}}{2 S_{21}} & z_{o} \frac{\left(1+S_{11}\right)\left(1+S_{22}\right)-S_{12} S_{21}}{2 S_{21}} \\
\frac{1}{Z_{o}} \frac{\left(1-S_{11}\right)\left(1-S_{22}\right)-S_{12} S_{21}}{2 S_{21}} & \frac{\left(1-S_{11}\right)\left(1+S_{22}\right)+S_{12} S_{21}}{2 S_{21}}
\end{array}\right]
$$


To obtain the chain matrix of the embedded line, the chain matrix of the long structure is multiplied by the inverse matrix of the short structure. This multiplication results in a new chain matrix $\left(\left[A_{L S}\right]\right)$ that facilitates the de-embedding process; $\left[A_{L S}\right]$ can be written as

$$
\begin{aligned}
{\left[A_{L S}\right] } & =\left[A_{L}\right] \cdot\left[A_{S}\right]^{-1} \\
& =\left[A_{P 1 L}\right] \cdot\left[A_{T L}\right] \cdot\left[A_{P 2 S}\right]^{-1}
\end{aligned}
$$

where $\left[A_{P I L}\right],\left[A_{T L}\right]$, and $\left[A_{P 2 S}\right]$, are the chain matrices of the input test pad of the long structure, the embedded transmission line section, and the output test pad of the short structure, respectively. With the assumption of identical test pads for the short and the long test structures (i.e., $\left[A_{P I L}\right]=\left[A_{P 2 S}\right]$ ), (3.15) can be expressed in terms of the pad's admittance $\left(Y_{P}\right)$, hence,

$$
\left[A_{L S}\right]=\left[\begin{array}{ll}
1 & 0 \\
Y_{P} & 1
\end{array}\right] \cdot\left[A_{T L}\right] \cdot\left[\begin{array}{lr}
1 & 0 \\
-Y_{P} & 1
\end{array}\right]
$$

The $\left[A_{L S}\right]$ matrix can be converted to an admittance-matrix $\left(\left[Y_{L S}\right]\right)$ in order to determine the admittance matrix of the embedded line $\left(\left[Y_{T L}\right]\right)$. $\left[Y_{T L}\right]$ can be calculated from the following equation:

$$
\left[Y_{T L}\right]=\frac{\left[\begin{array}{ll}
\left(Y_{L S}\right)_{11} & \left(Y_{L S}\right)_{12} \\
\left(Y_{L S}\right)_{21} & \left(Y_{L S}\right)_{22}
\end{array}\right]+\left[\begin{array}{ll}
\left(Y_{L S}\right)_{22} & \left(Y_{L S}\right)_{21} \\
\left(Y_{L S}\right)_{12} & \left(Y_{L S}\right)_{11}
\end{array}\right]}{2}
$$

Swapping the elements along the diagonal axis of $\left[Y_{L S}\right]$ in (3.17) removes the effect of the test pads. Having $\left[Y_{T L}\right]$, the chain matrix of the embedded line $\left(\left[A_{\mathrm{TL}}\right]\right)$ can be 
obtained and the associated characteristic impedance $\left(Z_{o}\right)$, attenuation factor, and phase constant can be calculated from the following equations:

$$
\begin{aligned}
Z_{o} & \left.=\sqrt{\frac{\left(A_{T L}\right)_{12}}{\left(A_{T L}\right)_{21}}}\right] \\
\alpha & =\operatorname{Re}\left[\frac{\cosh ^{-1}\left(A_{T L}\right)_{11}}{l}\right] \\
\beta & =\operatorname{Im}\left[\frac{\cosh ^{-1}\left(A_{T L}\right)_{11}}{l}\right] \\
\varepsilon_{\text {eff }} & =\left(\frac{0.3 \beta}{2 \pi \cdot f}\right)^{2}
\end{aligned}
$$




\subsection{Measurements and EM Simulation Results for CMOS LG-CPW Transmission Lines}

Figure 3.9 shows the electromagnetic simulation results of the characteristic impedances of the conventional CMOS CPW transmission lines of Table 3.1.

Table 3-1: Dimensions of conventional CPW transmission line

\begin{tabular}{|c|c|c|c|c|}
\hline Line \# & Width (um) & Space (um) & Ground Width (um) & Metal Layer \\
\hline L1 & 12 & 2 & 12 & M9 \\
\hline L2 & 3 & 6 & 12 & M9 \\
\hline L3 & 10 & 2.5 & 12 & M9 \\
\hline L4 & 3 & 2 & 12 & M9 \\
\hline
\end{tabular}

The maximum allowable metal-width of the $90-\mathrm{nm}$ CMOS process which is used in this work is $12 \mu \mathrm{m}$, the minimum space is $2 \mu \mathrm{m}$ (i.e., spacing between the edges of the signal-line and the ground strips), and the minimum width is $3 \mu \mathrm{m}$. The distance between the bottom of the signal-conductor on M9 and the top of the Si-substrate is $7 \mu \mathrm{m}$. The transmission lines $\mathrm{L} 1$ and $\mathrm{L} 2$ provide the minimum and maximum impedance that can be obtained with this process, whereas L3 and L4 provide similar impedances of 50 ohms. From Figure 3.9, the dimensions of L1 resulted in a characteristic impedance of $42 \mathrm{ohms}$, this is considered the lowest impedance that can be achieved in this process without violating the technology design rules. On the other hand, L2 provided a characteristic impedance of 81 , ohms which is considered the highest impedance that can be obtained with low loss. L3 and L4 provided similar characteristic impedances of 50 ohms. Although L4 occupies smaller area compared to $\mathrm{L} 3$, the resistance/unit-length of $\mathrm{L} 3$ is higher which leads to a higher attenuation. 
Also, L4 can not carry current more than $6 \mathrm{~mA}$ according to the process design rules of $2 \mathrm{~mA} / \mu \mathrm{m}$.

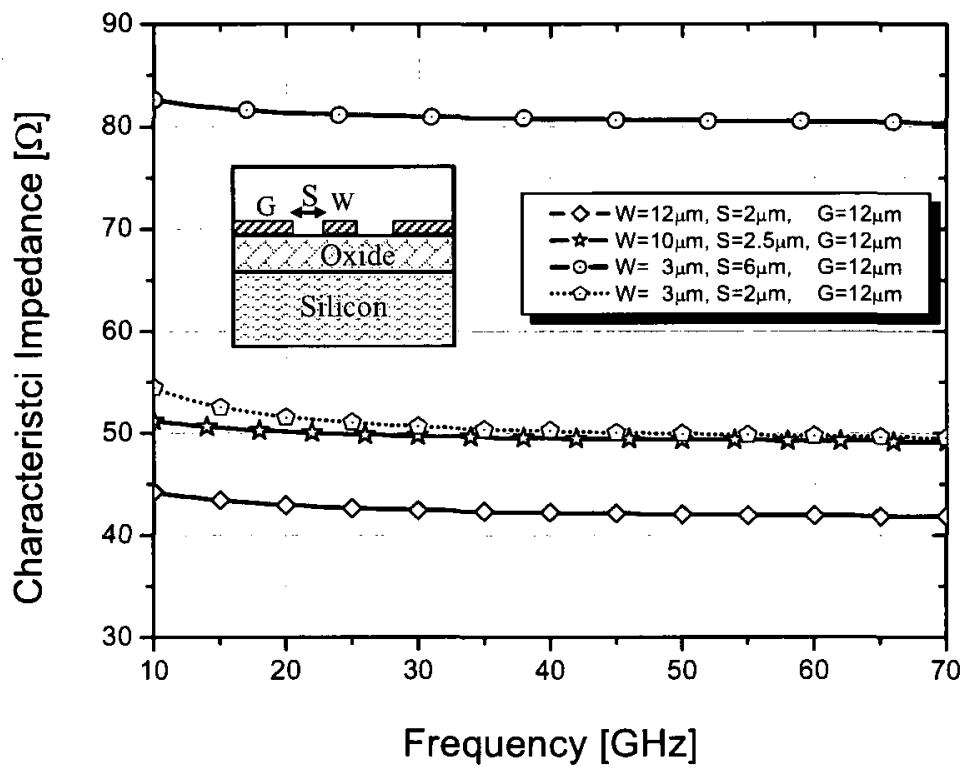

Figure 3.9: Characteristic impedances of CMOS CPW transmission lines of different dimensions

Despite lower impedances being achievable by increasing the width of the signal-line, the design rules require introducing slots in the line if the width is greater than $12 \mu \mathrm{m}$, the number of slots and the slots size are defined by the process design rules. However, having slots in the signal-conductor increases its characteristic impedance. On the other hand, higher impedances can be realized by using the minimum metalwidth $(3 \mu \mathrm{m})$ or increasing the gap between the signal-line and the ground strips; but reducing the width of the signal-line increases its resistance which increases the attenuation. Also, it limits the current path through the line. Figure 3.10 shows the resistance/mm for two $50 \mathrm{ohms} \mathrm{CPW}$ lines of different signal-line widths $(\mathrm{W}=3 \mu \mathrm{m}$, 
and $10 \mu \mathrm{m})$. From Figure 3.10 , at $60 \mathrm{GHz}$ the narrow line $(\mathrm{W}=3 \mu \mathrm{m})$ demonstrates a resistance of $12 \mathrm{ohms} / \mathrm{mm}$ while the wider line $(\mathrm{W}=10 \mu \mathrm{m})$ demonstrates a resistance of $8.2 \mathrm{ohms} / \mathrm{mm}$. The increase in the resistance leads to a higher attenuation as shown in Figure 3.11. From Figure 3.11, the wide line $(\mathrm{W}=10 \mu \mathrm{m})$ has an attenuation of 0.83 $\mathrm{dB} / \mathrm{mm}$ whereas the narrow line $(\mathrm{W}=3 \mu \mathrm{m})$ has an attenuation of $1.5 \mathrm{~dB} / \mathrm{mm}$ which corresponds to an increase of $16 \%$ in the attenuation of the narrow line.

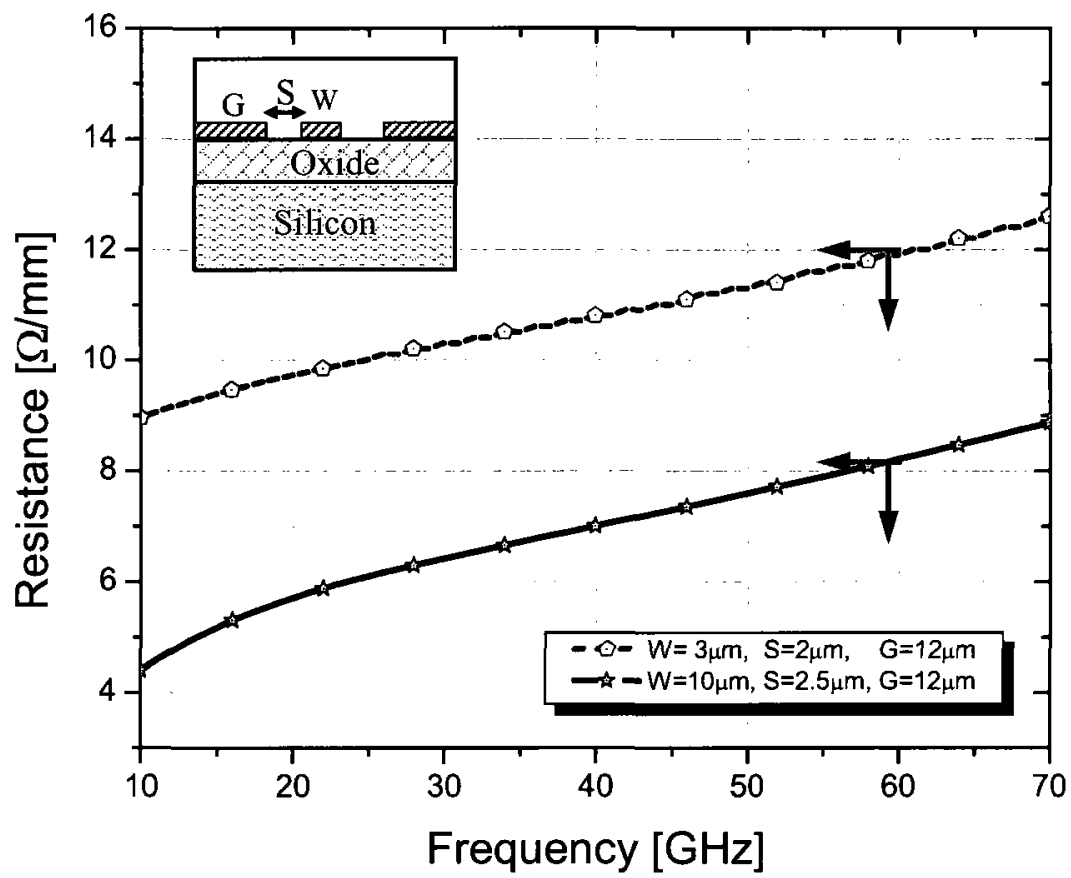

Figure 3.10: Resistance per unit-length for two 50 CMOS CPW transmission lines of different dimensions 


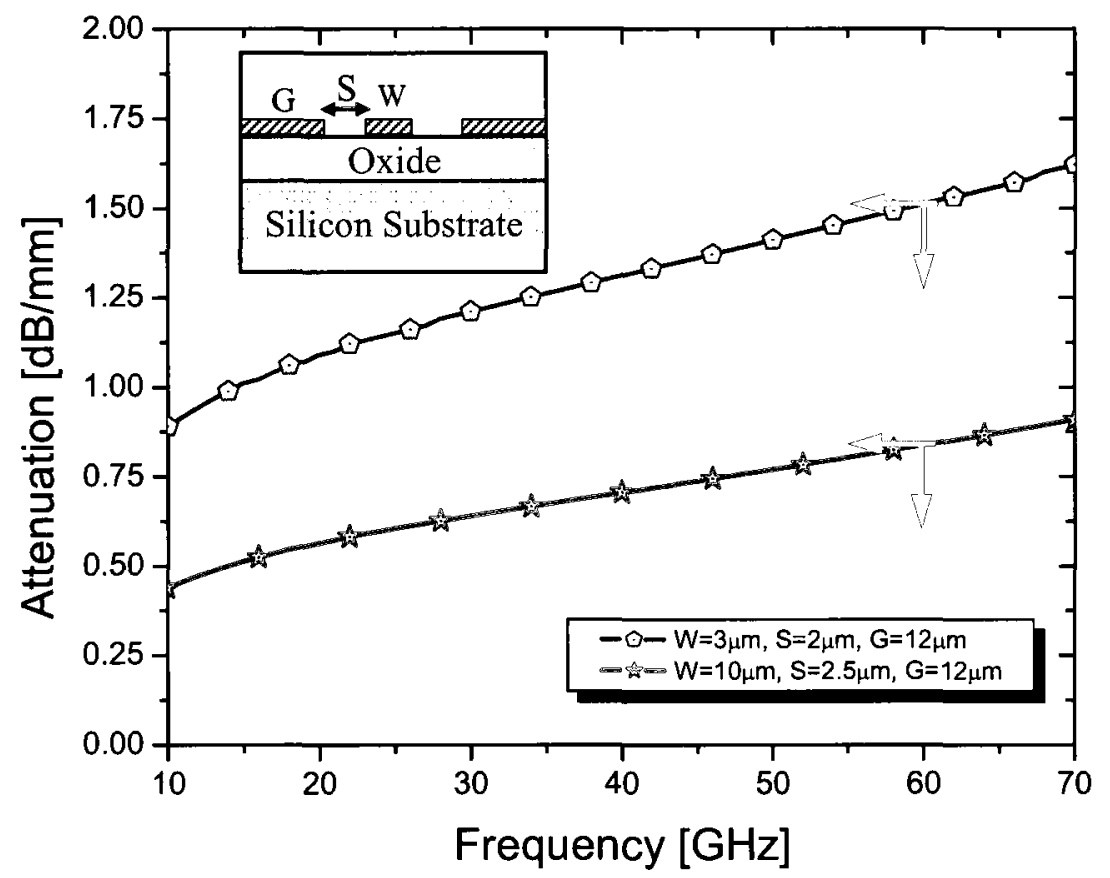

Figure 3.11: Attenuation factor of two CPWs with different dimensions

To validate the EM simulation results, a set of CPW test structures of $W=10 \mu \mathrm{m}$, $\mathrm{S}=2.5 \mu \mathrm{m}, \mathrm{G}=12 \mu \mathrm{m}$ was fabricated on the top metal-layer in a $90-\mathrm{nm}$ CMOS process with 9-metal layers named M1-M9 from bottom to top. Figure 3.12 shows the microphotograph of the short and long test structures that are used to extract the measured parameters of the transmission line.

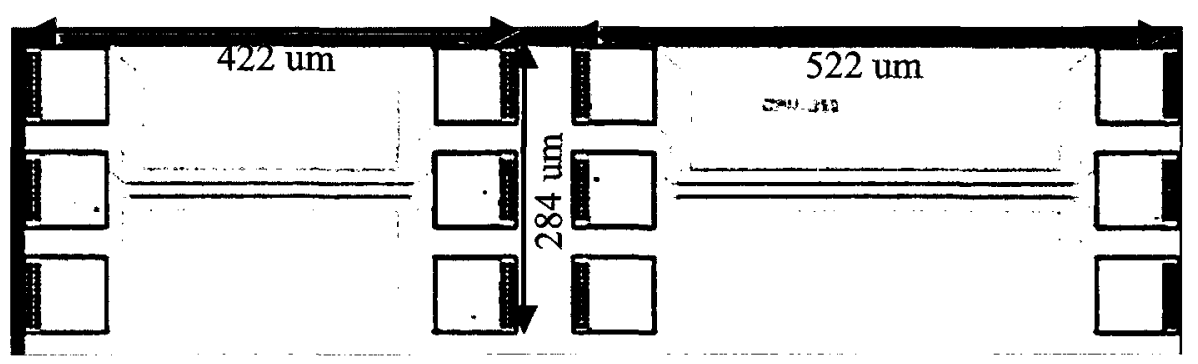

Figure 3.12: Microphotograph of short and long CPW test structures implemented on metal-9 in a 90nm 9-metal layers CMOS process. 
Figure 3.13 shows the extracted measurements and the EM simulation results of the characteristic impedance of a CPW transmission line with $\mathrm{W}=10 \mu \mathrm{m}, \mathrm{G}=12 \mu \mathrm{m}$, and $\mathrm{S}=2.5 \mu \mathrm{m}$ and implemented on M9 which has thickness of $0.81 \mu \mathrm{m}$. From Figure 3.13 both the measurements and simulation results agree very well.

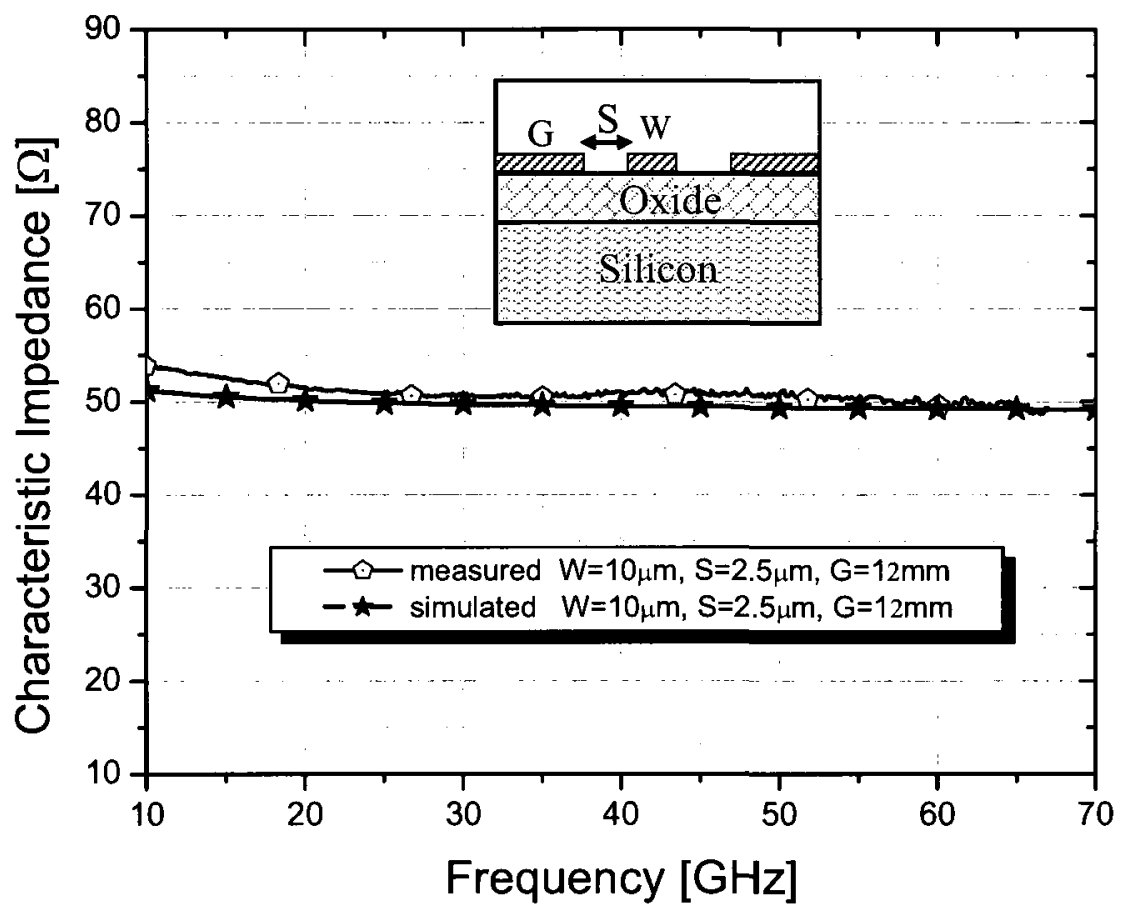

Figure 3.13: Extracted and simulated characteristic impedances of a CMOS CPW transmission line on $\mathrm{M} 9(\mathrm{~W}=10 \mu \mathrm{m}, \mathrm{S}=2.5 \mu \mathrm{m}, \mathrm{G}=12 \mu \mathrm{m}, \mathrm{t}=0.81 \mu \mathrm{m})$

The extracted and simulated attenuation $/ \mathrm{mm}$ of the $50 \mathrm{ohms}$ CPW line are shown in Figure 3.14, from Figure 3.14 the measured attenuation agrees reasonably well with the simulation results over the frequency range of $30-64 \mathrm{GHz}$. 


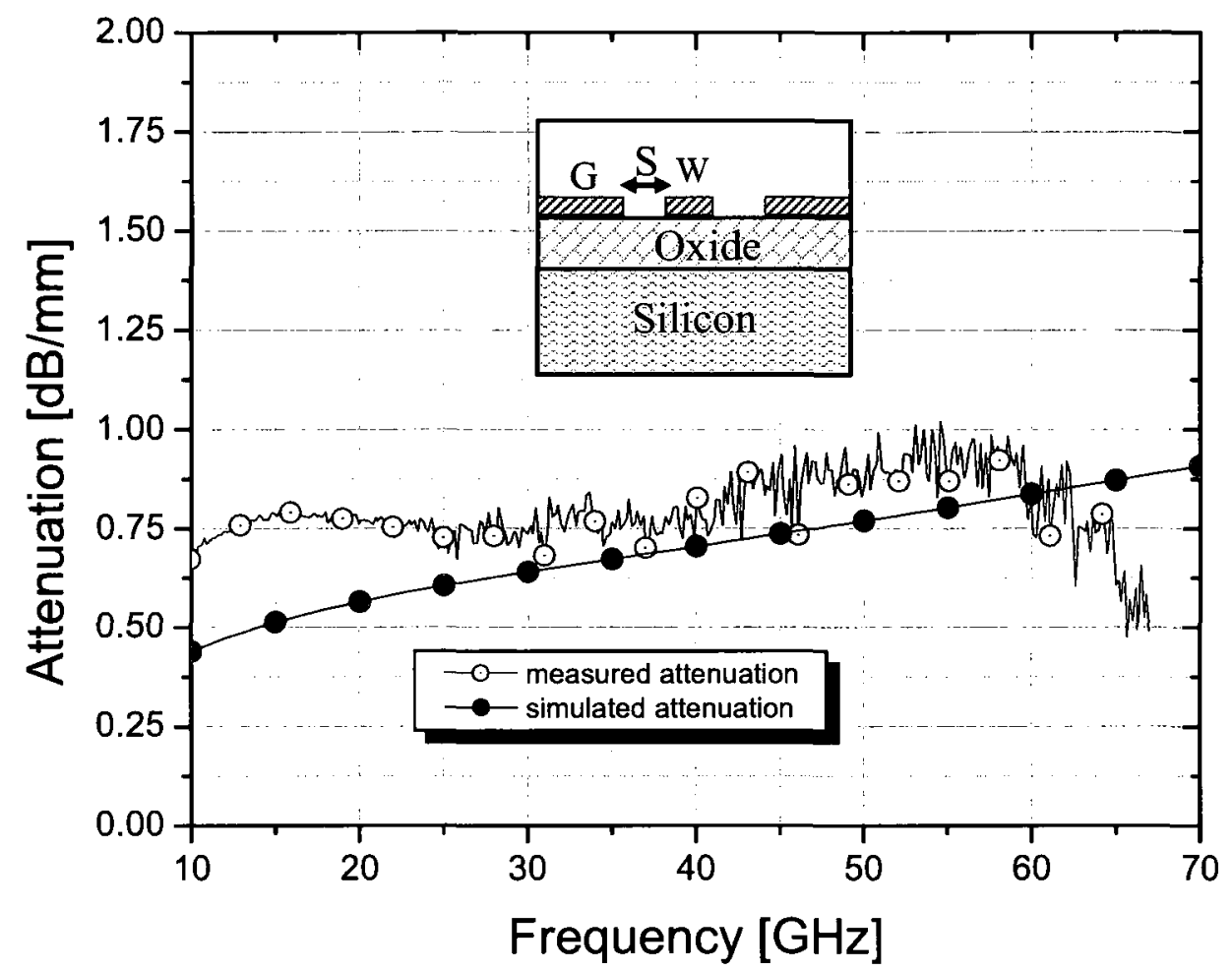

Figure 3.14: Extracted and simulated attenuation of a $50 \mathrm{Ohms}$ CPW transmission line on $\mathrm{M} 9(\mathrm{~W}=10 \mu \mathrm{m}, \mathrm{S}=2.5 \mu \mathrm{m}, \mathrm{G}=12 \mu \mathrm{m}, \mathrm{t}=0.81 \mu \mathrm{m})$

Figure 3.15 shows the extracted and simulated effective permittivity of the $50 \mathrm{ohms}$ CMOS CPW line. From Figure 3.15 the extracted and simulated data agree reasonably over the frequency range of $55-67 \mathrm{GHz}$.

The measurements of the $50 \mathrm{ohms} \mathrm{CPW}$ line are used as base-line measurements for evaluating the performance of the lower-ground $\mathrm{CPW}$ transmission lines of this study. Figure 3.16 shows the EM simulation results of the characteristic impedance of LG-CPW transmission lines of different heights (the height of the signal-line relative to the ground strips). From Figure 3.16, the line which has ground strips on M1 provided a characteristic impedance of $61 \mathrm{ohms}$ compared to $50 \mathrm{ohms}$ for the line of 
the same dimensions but with its ground strips on M9. Therefore, lowering the ground strips by $6.38 \mu \mathrm{m}$ (i.e., distance between M9 and M1) resulted in an increase of almost $25 \%$ in the characteristic impedance compared to a $50 \mathrm{ohms}$ CPW line.

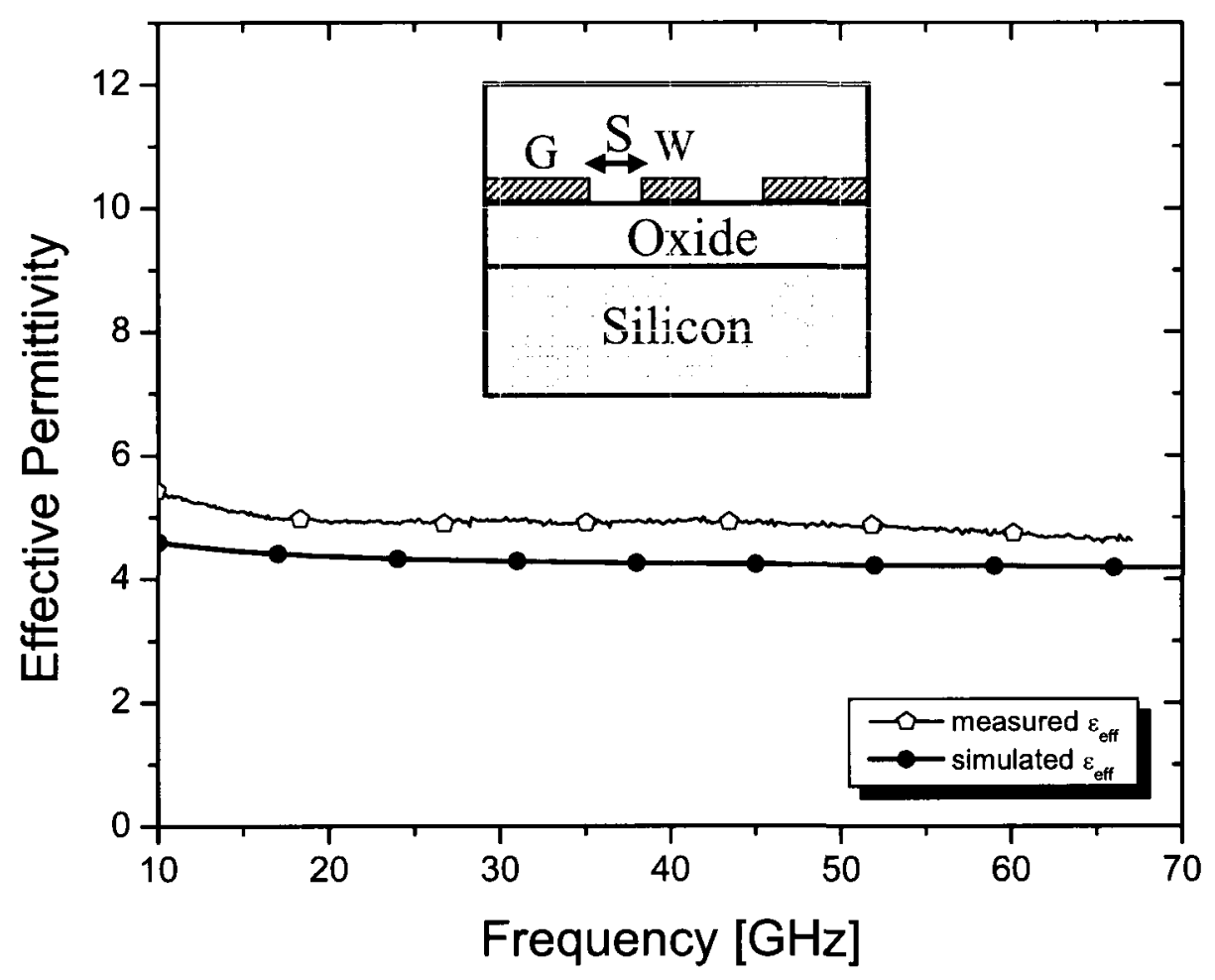

Figure 3.15: Extracted and simulated effective permittivity of a $50 \mathrm{Ohms}$ CMOS CPW transmission line on $\mathrm{M} 9(\mathrm{~W}=10 \mu \mathrm{m}, \mathrm{S}=2.5 \mu \mathrm{m}, \mathrm{G}=12 \mu \mathrm{m}, \mathrm{t}=0.81 \mu \mathrm{m})$ 


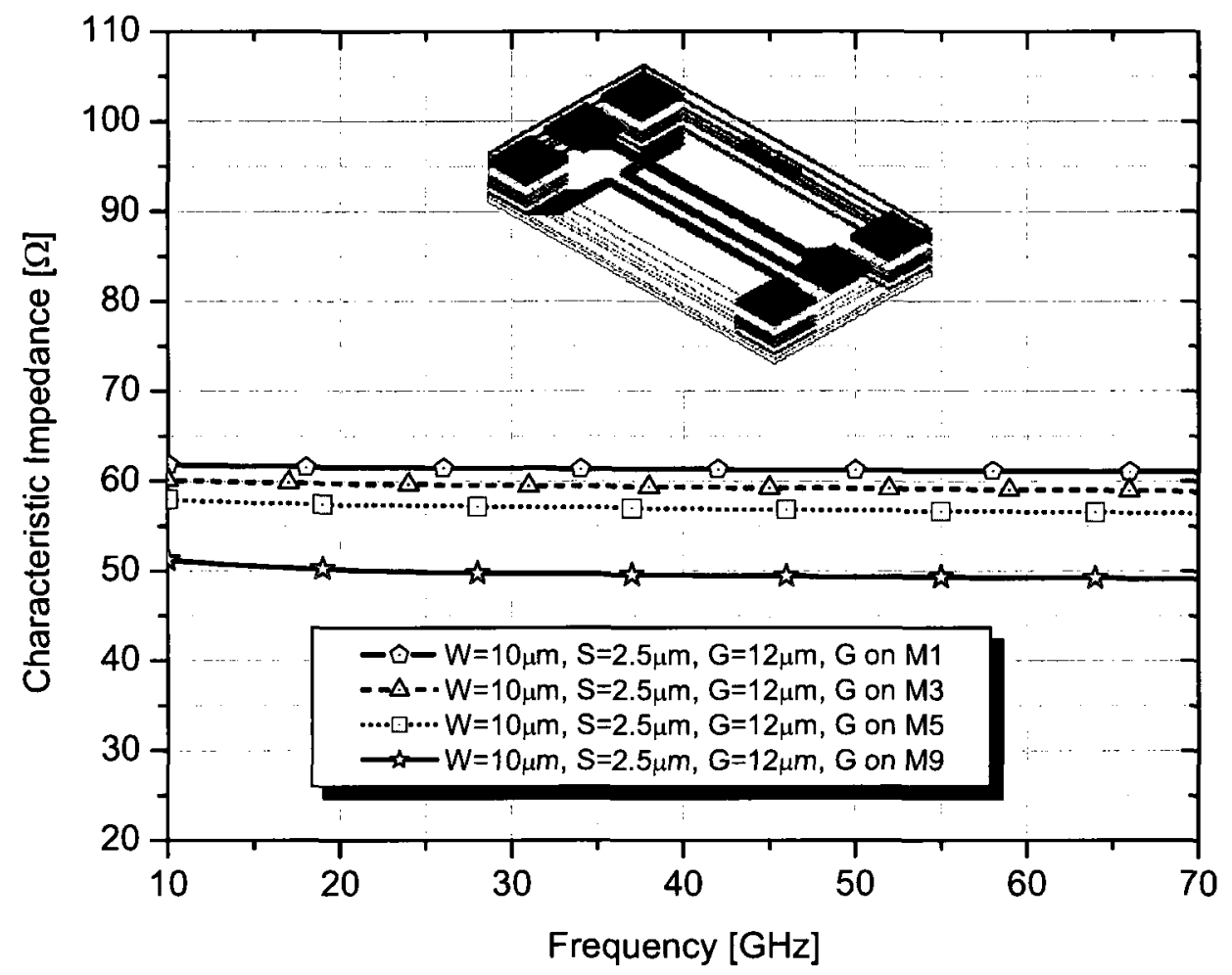

Figure 3.16: Characteristic impedances of LG-CPW transmission lines of different heights

Figure 3.17 shows the extracted and simulated characteristic impedances of a CPW line on M9 and a LG-CPW line of the same size but with its ground strips on M3M2. M3 and M2 were combined through vias to increase the thickness of the ground plane $(\mathrm{M} 2+\mathrm{M} 3+\mathrm{IMD} 2=0.82 \mu \mathrm{m})$. 


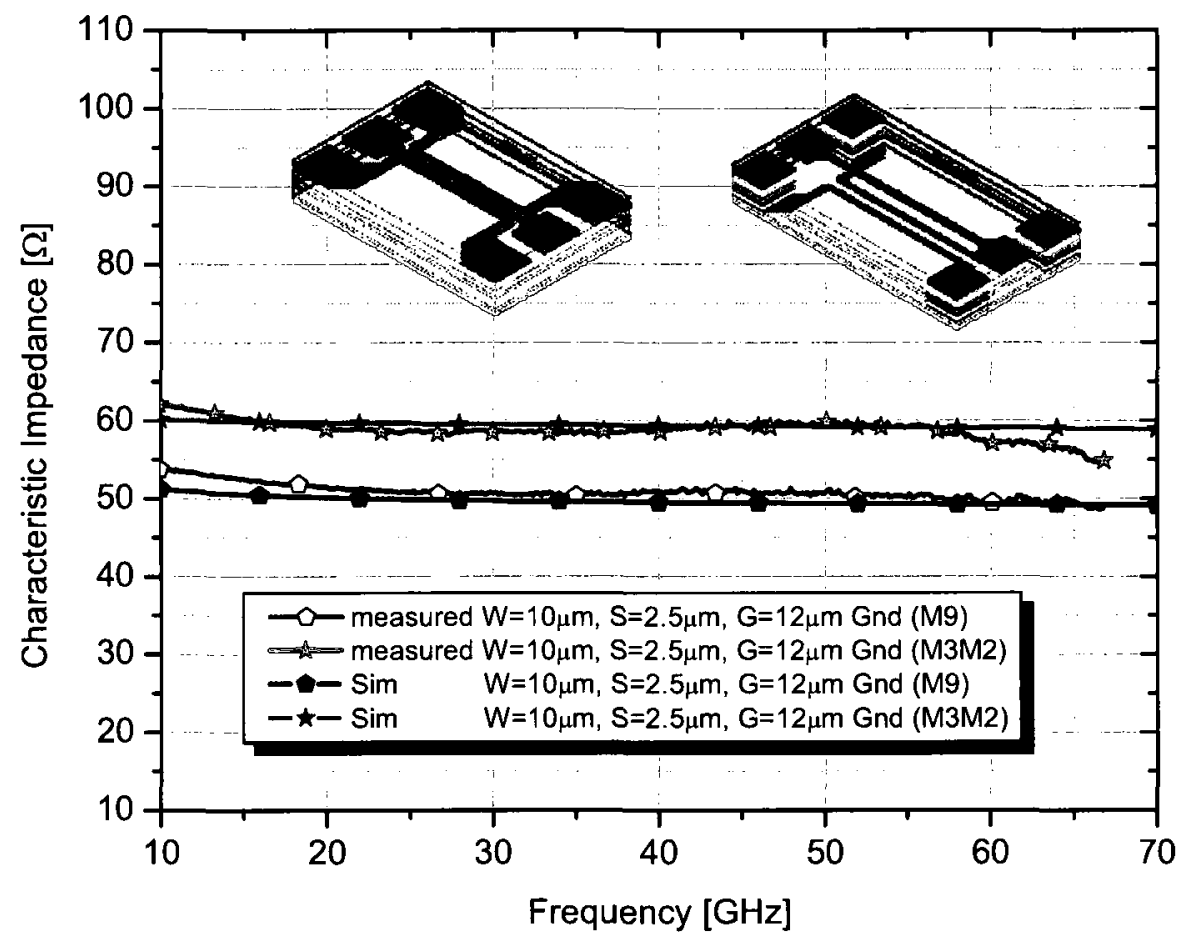

Figure 3.17: Extracted and simulated characteristic impedances of CMOS LG-CPW and CPW transmission lines

From Figure 3.17, the measured and simulated results of the CPW structure agree very well over the frequency range of $20-67 \mathrm{GHz}$ (the vector network analyzer which is used in this measurements operates up to $67 \mathrm{GHz}$ ). The measured and simulated impedances of the LG-CPW structure agree well over the frequency range of 10-59 $\mathrm{GHz}$, and reasonably agree over the frequency range of $59-65 \mathrm{GHz}$. The extracted attenuation factors of both the CPW and the LG-CPW (ground on M3:M2) transmission lines are shown in Figure 3.18. 


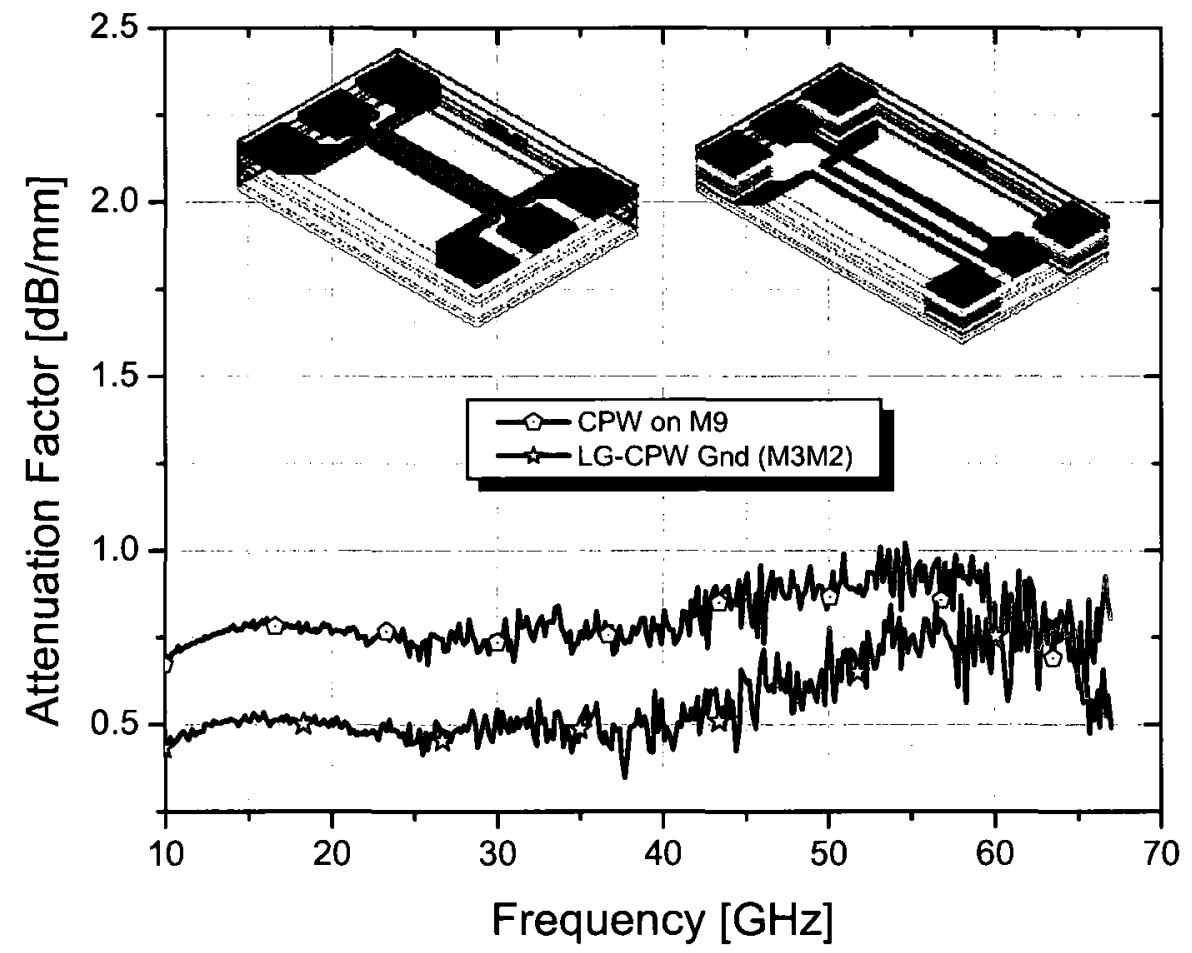

Figure 3.18: Extracted attenuation/mm of CMOS LG-CPW and CPW transmission lines.

From Figure 3.18, the attenuation factor of the LG-CPW is slightly lower than that of the CPW, such a decrease in the attenuation is attributed to the reduced current crowding at the edges of the signal-line. The extracted effective permittivity of the CPW and LG-CPW are shown in Figure 3.19. From Figure 3.19, the change in the $\varepsilon_{\mathrm{eff}}$ over the frequency range of $55-67 \mathrm{GHz}$ is insignificant for the LG-CPW transmission line. This indicates that the LG-CPW has very good dispersion performance. A microphotograph of the test structures of both the CPW and the LG-CPW is shown in Figure 3.20. 


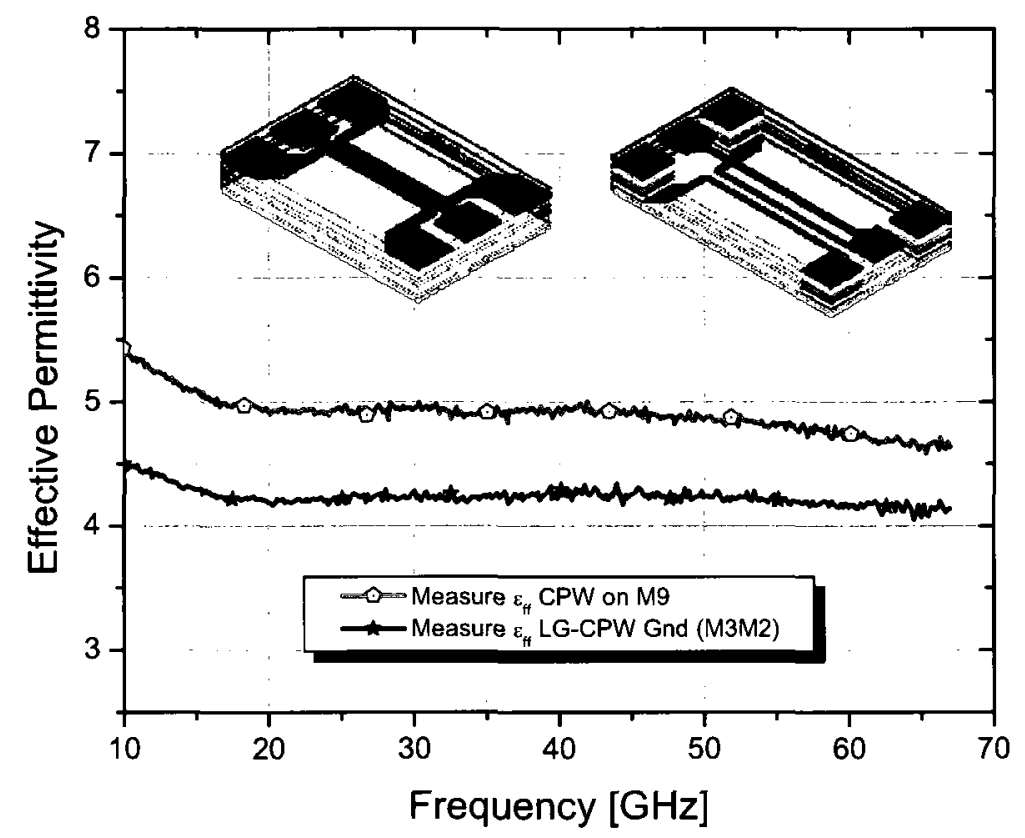

Figure 3.19: Extracted effective permittivity of CMOS LG-CPW and CPW transmission lines

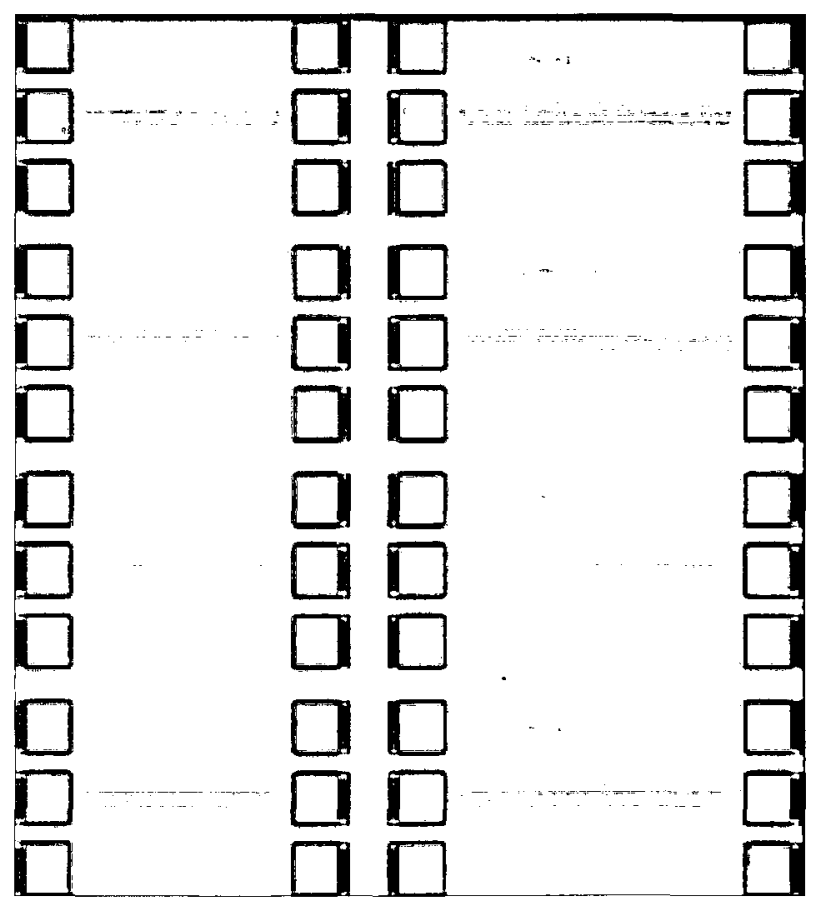

Figure 3.20: Microphotograph of short and long CPW and LG-CPW test structures implemented in a $90 \mathrm{~nm} 9$-metal layers CMOS process. 
The EM simulation results of the maximum and minimum impedances that can be obtained using LG-CPW in this 90-nm RF CMOS process are shown in Figure 3.21.

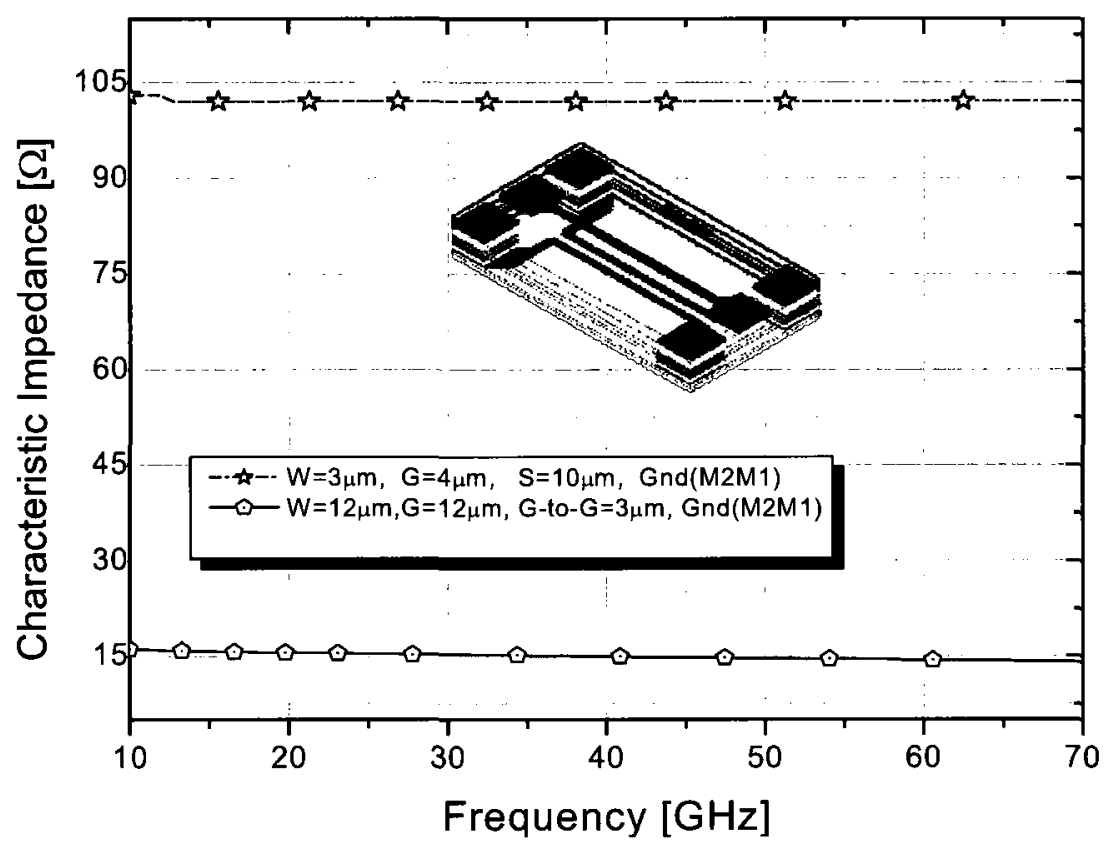

Figure 3.21: Simulated extreme characteristic impedance of LG-CPW in a $90-\mathrm{nm}$ CMOS process

From Figure 3.21, the maximum impedance that can be obtained with LG-CPW in this process is $104 \mathrm{ohms}$, this can be realized with a line which has $\mathrm{W}=3 \mu \mathrm{m}, \mathrm{G}=4 \mu \mathrm{m}$, $\mathrm{S}=10 \mu \mathrm{m}$ with the ground strips implemented on M2 and M1 $(\mathrm{M} 1+\mathrm{IMD} 1+\mathrm{M} 2=0.79 \mu \mathrm{m})$. Also, the minimum impedance that can be obtained is 15 ohms and can be realized by using vertical LG-CPW of $\mathrm{W}=12 \mu \mathrm{m}, \mathrm{G}=12 \mu \mathrm{m}$, G-to$\mathrm{G}=3 \mu \mathrm{m}$, and the ground strips are implemented on M8M7M6 $(\mathrm{M} 6+\mathrm{IMD} 6+\mathrm{M} 7+\mathrm{IMD} 7+\mathrm{M} 8=2.05 \mu \mathrm{m})$. From Figure 3.21 , the impedance range of 
LG-CPW is wider than that of the conventional CPW. Hence, LG-CPW can provide design flexibility for the design of $\mathrm{mmW}$ integrated circuits. Also, they enable the realization of high impedance lines for size-reduction of passive elements using capacitive loading.

\subsection{Chapter Summary}

In this chapter, CMOS CPW and LG-CPW transmission lines were analyzed, experimentally characterized, and simulated using an electromagnetic simulator. Based on the characterization and simulation results of these transmission lines, the CMOS LG-CPW transmission lines can provide a wide range of impedances from 15$104 \mathrm{ohms}$ in a $90 \mathrm{~nm}$ CMOS process with 9-metal layers. Furthermore, the LG-CPW provides design flexibility to mitigate the geometry limitations that are imposed by the CMOS design rules. The LG-CPW transmission lines of this chapter will be used in the following chapters to develop a V-band CMOS low noise amplifier, and a reduced-size $60-\mathrm{GHz}$ band quadrature coupler for $\mathrm{CMOS}$ radio transceiver frontends. 


\section{Chapter 4}

\section{Design of a V-band Si-based LNA using LG-CPW Transmission Lines}

This chapter focuses on the utilization of the lower-ground coplanar waveguide (LGCPW) transmission lines which were studied in Chapter 3 to design a $57-\mathrm{GHz}$ lownoise, high-gain, high linearity single-stage amplifier in a 90-nm RF-CMOS technology. The design methodology and the LNA performance in terms of noise figure, gain, linearity, and power consumption are also presented and discussed. Experimental measurements and post-layout EM simulation results are presented and compared.

\subsection{Motivation}

Most of today's wireless systems for consumer electronic applications use $2.4 \mathrm{GHz}$ and $5 \mathrm{GHz}$ frequency bands. However, the growing demand for high data rates necessitates the development of low-cost integrated systems at $\mathrm{mmW}$ frequencies where a significantly large bandwidth is available. Furthermore, wireless transmission using $\mathrm{mmW}$ frequencies reduces the size of the antennas and their spacing in a multiple antenna scheme, making phased-array antenna systems practical for portable communications systems. Figure 4.1 shows a simplified block diagram of a V-band band transceiver front-end for high data rate radio-over-fiber [59]-[62] applications. In 
mmW systems, the low-noise amplifiers (LNA) is one of the most important building blocks because it is the first stage in the receiver's chain of the system and influences the receiver's noise figure; thus, the LNA has significant impacts on the system's link budget and bit-error-rate. 


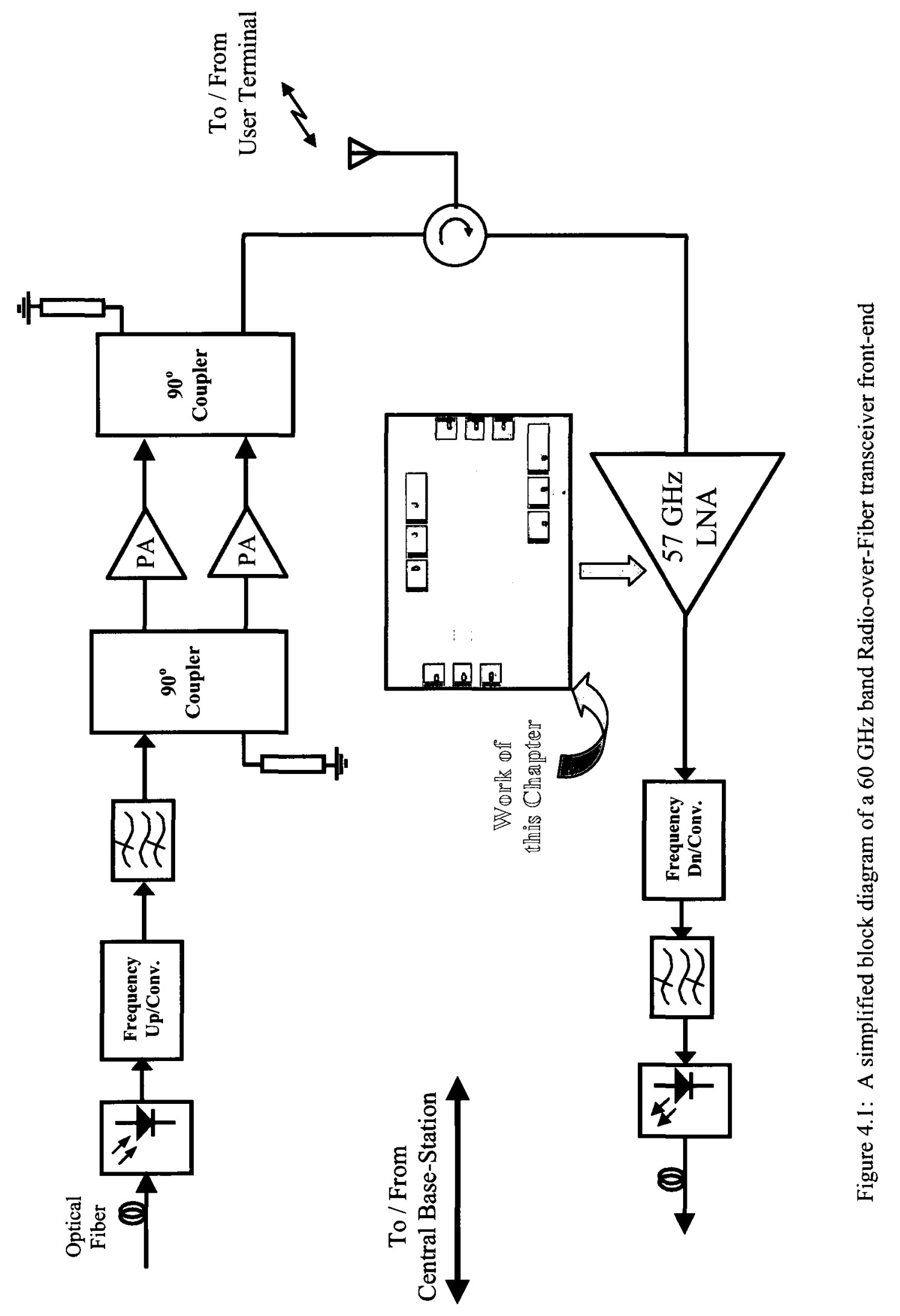


Si-based CMOS technology is very attractive for the development of $\mathrm{mmW}$ systems [63]-[68] because of its potential for integrating both the RF and DSP functions; also, because of its low-cost. Although CMOS technology enables the design of low-cost fully integrated mmW systems, the design rules of the CMOS technology could present challenges for having optimal designs. The CMOS design rules of maximum/minimum metal width and the minimum metal spacing, limit the range of impedances that can be realized to design optimal integrated circuits. The design rule limitations of CMOS technology motivate the design effort of this research to develop a V-band CMOS low noise amplifier [69]-[74], which provides low-noise figure, high gain, high linearity, and low power consumption.

\subsection{Noise Figure of a Low Noise Amplifier}

The noise figure (NF) of low-noise amplifiers in $\mathrm{mmW}$ receivers is the most important design parameter and impacts the receiver's sensitivity, which affects the bit-error-rate (BER) of the radio link and the communication range. Improving the receiver sensitivity also relaxes the specifications on the transmit power and inhances the system's link budget. The NF of a two-port amplifier [30] can be expressed as:

$$
N F=N F_{\min }+\frac{R_{n}}{G_{s}}\left|Y_{s}-Y_{o p t}\right|^{2}
$$

where,

$$
Y_{s}=G_{s}+B_{s},
$$

$Y_{s}$ is the admittance presented to the active device, $Y_{o p t}$ is the optimum admittance for minimum noise figure, $N F_{\min }$ is the minimum noise figure of the device (at $Y_{s}=Y_{o p t}$ ), 
and $R_{\mathrm{n}}$ is the equivalent noise resistance of the active device. The admittances $Y_{s}$ and $Y_{o p t}$ can be expressed in terms of the reflection coefficients $\Gamma_{\mathrm{s}}$ and $\Gamma_{\mathrm{opt}}$, thus,

$$
\begin{gathered}
Y_{s}=\frac{1}{Z_{o}} \frac{1-\Gamma_{s}}{1+\Gamma_{s}} \\
Y_{o p t}=\frac{1}{Z_{o}} \frac{1-\Gamma_{o p t}}{1+\Gamma_{o p t}}
\end{gathered}
$$

Substituting (4.3) and (4.4) into (4.1), the NF can be written as

$$
N F=N F_{\min }+\frac{4 R_{n}}{Z_{o}} \frac{\left|\Gamma_{s}-\Gamma_{o p t}\right|^{2}}{\left(1-\left|\Gamma_{s}\right|^{2}\right)\left|1+\Gamma_{o p t}\right|^{2}}
$$

The parameters $N F_{\min }, \Gamma_{\mathrm{opt}}$, and $R_{\mathrm{n}}$ are the noise parameters of the device and are given by the foundry, or measured. Eq. (4.5) points out that to have a low noise figure, the intrinsic resistance of the device must be small.

\subsection{Impact of the Transistor Size on Noise-Figure}

One of the major issues during the design of a LNA is determining the proper transistor size (i.e., the finger-width, and the number of fingers), which provides minimum noise figure $\left(N F_{\min }\right)$ and optimal gain $\left(G_{\max }\right)$. Since it is impossible to have both of the minimum $N F$ and maximum gain, some trade-off must be done to obtain the best system performance. Figure 4.2 shows the simulated $N F_{\min }$ as a function of the number

of fingers for different finger widths $\left(V_{\mathrm{GS}}=0.7 \mathrm{~V}\right.$ and $\left.V_{\mathrm{DS}}=1.5 \mathrm{~V}\right)$. In Figure 4.2 , the 
$N F_{\min }$ decreases with the increase in the number of fingers which leads one to believe that more fingers result in a better noise performance. However, too many fingers increases the input capacitance of the device which reduces both the unity-current-gain frequency $\left(f_{\mathrm{T}}\right)$ and the unity-power-gain frequency $\left(f_{\max }\right)$ of the device. $f_{\mathrm{T}}$ and $f_{\max }$ can be expressed as:

$$
f_{T}=\frac{g_{m}}{2 \pi\left(C_{g s}+C_{g d}\right)}, \quad f_{\max }=\frac{f_{T}}{2} \sqrt{\frac{r_{d s}}{r_{g}}}
$$

where $g_{\mathrm{m}}$ is the transconductance, $C_{\mathrm{gs}}$ is the gate-source capacitances, $C_{\mathrm{gd}}$ is the gatedrain capacitances, $r_{d s}$ is drain-source resistance, and $r_{g}$ is the gate resistance.

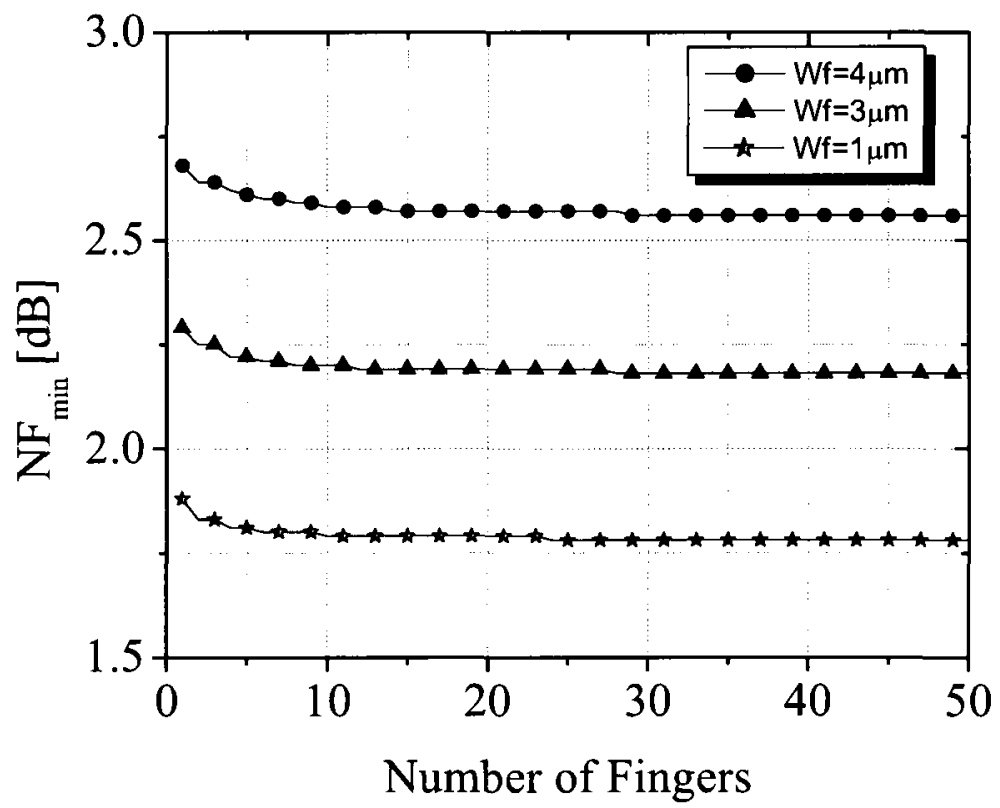

Figure 4.2: Minimum noise figure vs. the number of fingers for three different finger widths $\left(\mathrm{V}_{\mathrm{GS}}=0.7 \mathrm{~V}, \mathrm{~V}_{\mathrm{DS}}=1.5 \mathrm{~V}\right)$ at $57 \mathrm{GHz}$ 
From Figure 4.2, increasing the finger width degrades the noise figure, this is due to the increase of the noise resistance. Figure 4.3 shows the simulation results of the equivalent noise resistance of the device as a function of the number of fingers; increasing the number of fingers reduces the noise resistance which improves the noise figure. Figure 4.4 shows the $N F_{\min }$ as function of frequency for different finger widths. From Figure 4.4, with a finger width of $1 \mu \mathrm{m}$ the $N F$ of the device is approximately $1.7 \mathrm{~dB}$ at $60 \mathrm{GHz}$, whereas with a finger width of $3 \mu \mathrm{m}$ the $N F$ is $2.2 \mathrm{~dB}$. The device's finger width also impacts $f_{\max }$ of the device as shown in Figure 4.5. From Figure 4.5, using a finger width of $1 \mu \mathrm{m}$ resulted in a $f_{\max }=290 \mathrm{GHz}$, whereas using a finger width of $3 \mu \mathrm{m}$ resulted in a $f_{\max }=184 \mathrm{GHz}$. The obtained results of $f_{\max }$ are unrealistic and point out that the transistor's model is inaccurate. 


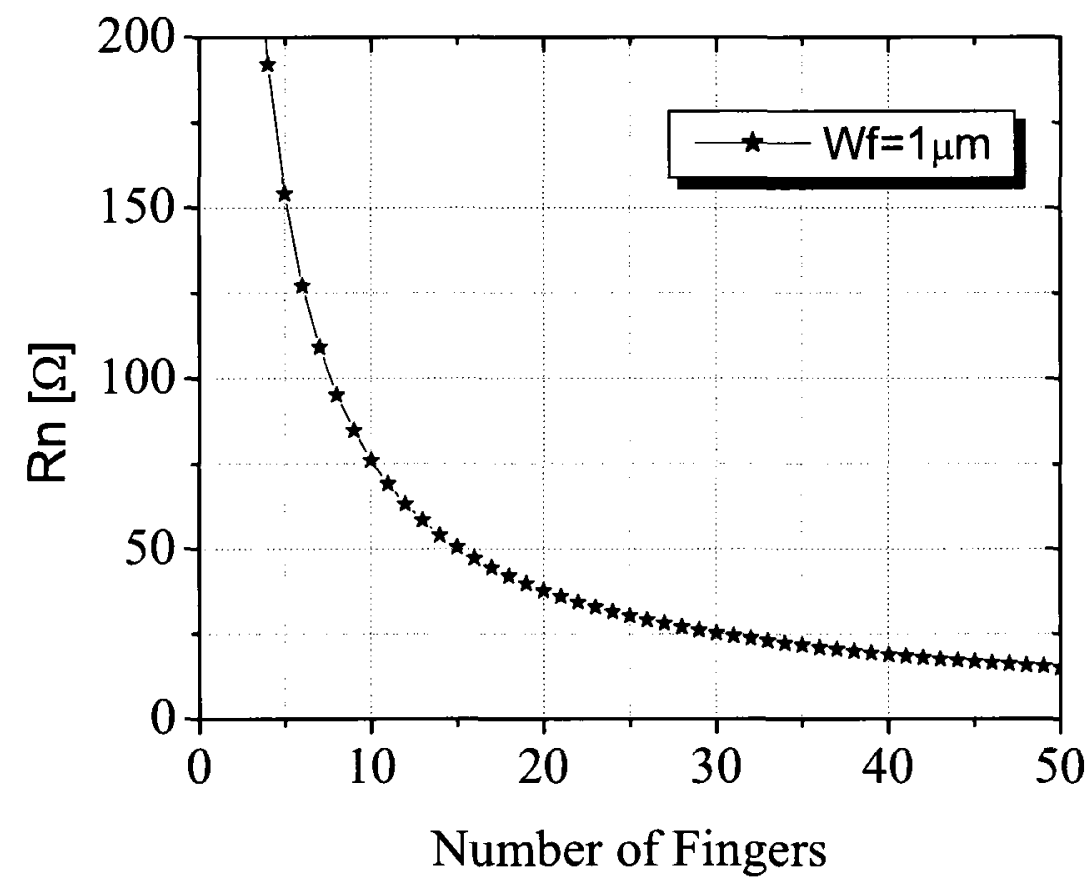

Figure 4.3: Equivalent noise resistance vs. number of fingers $\left(\mathrm{W}_{\mathrm{f}}=1 \mu \mathrm{m}, \mathrm{V}_{\mathrm{GS}}=0.7 \mathrm{~V}\right.$, $\mathrm{V}_{\mathrm{DS}}=1.5 \mathrm{~V}$ ) at $57 \mathrm{GHz}$.

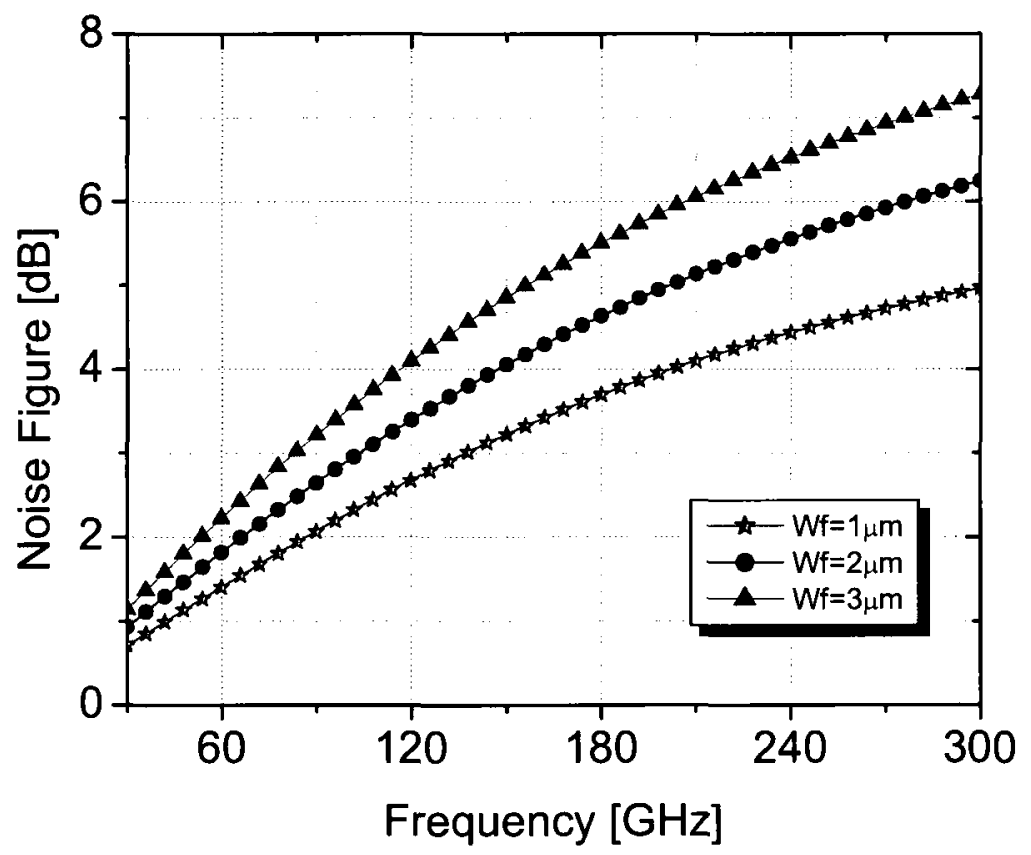

Figure 4.4: $\mathrm{NF}_{\min }$ vs. frequency for different device finger widths $\left(\mathrm{V}_{\mathrm{GS}}=0.7 \mathrm{~V}\right.$, $\left.\mathrm{V}_{\mathrm{DS}}=1.5 \mathrm{~V}\right)$ 


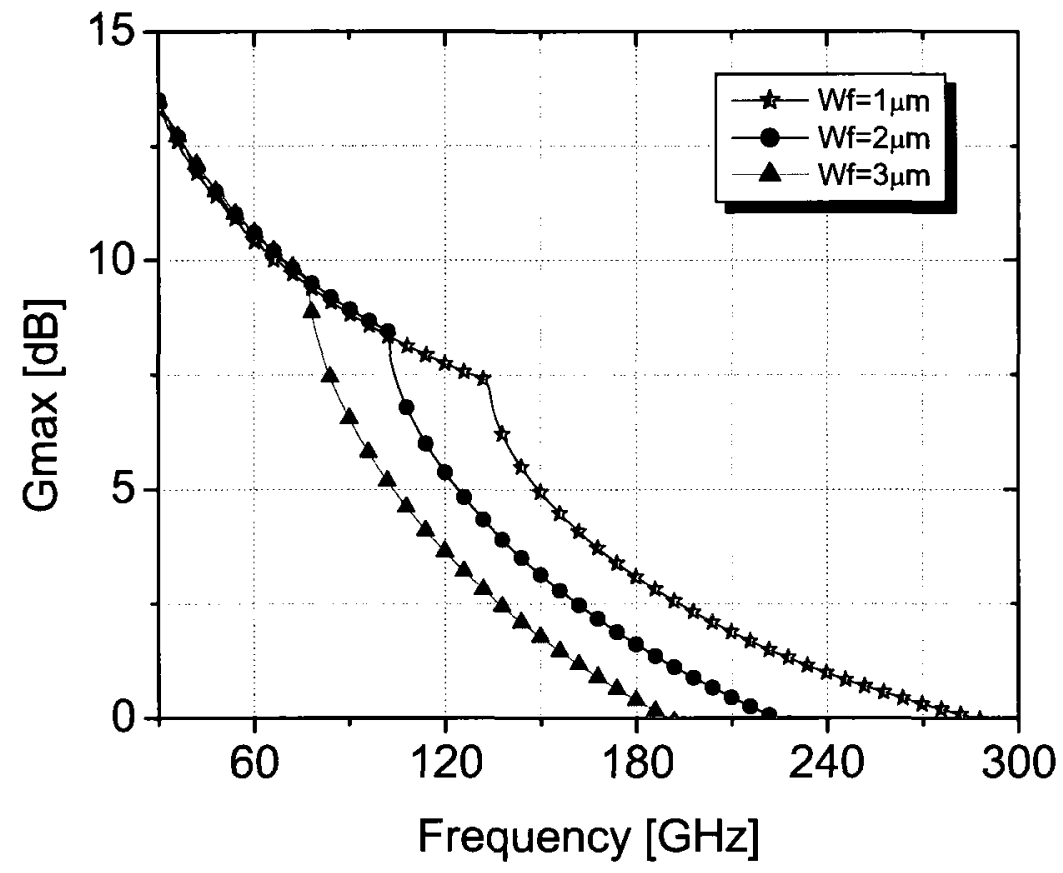

Figure 4.5: $f_{\max }$ for different finger widths $\left(\mathrm{V}_{\mathrm{GS}}=0.7 \mathrm{~V}, \mathrm{~V}_{\mathrm{DS}}=1.5 \mathrm{~V}\right)$

In this study, the device characterization for different finger widths and number of fingers resulted in the selection of a device size of 30 fingers with a finger width of $1 \mu \mathrm{m}$, the selected device is used as a common source (CS) device in the amplifier design of this study. The simulated I-V curves of the selected device are shown in Figure 4.6. From the $I-V$ curves, the device operating point $Q$ is chosen to have a current-draw less than $12 \mathrm{~mA}$ from a $1.5 \mathrm{~V}$ supply $\left(V_{\mathrm{GS}}=0.7 \mathrm{~V}\right.$ and $\left.V_{\mathrm{DS}}=1.5 \mathrm{~V}\right)$. 


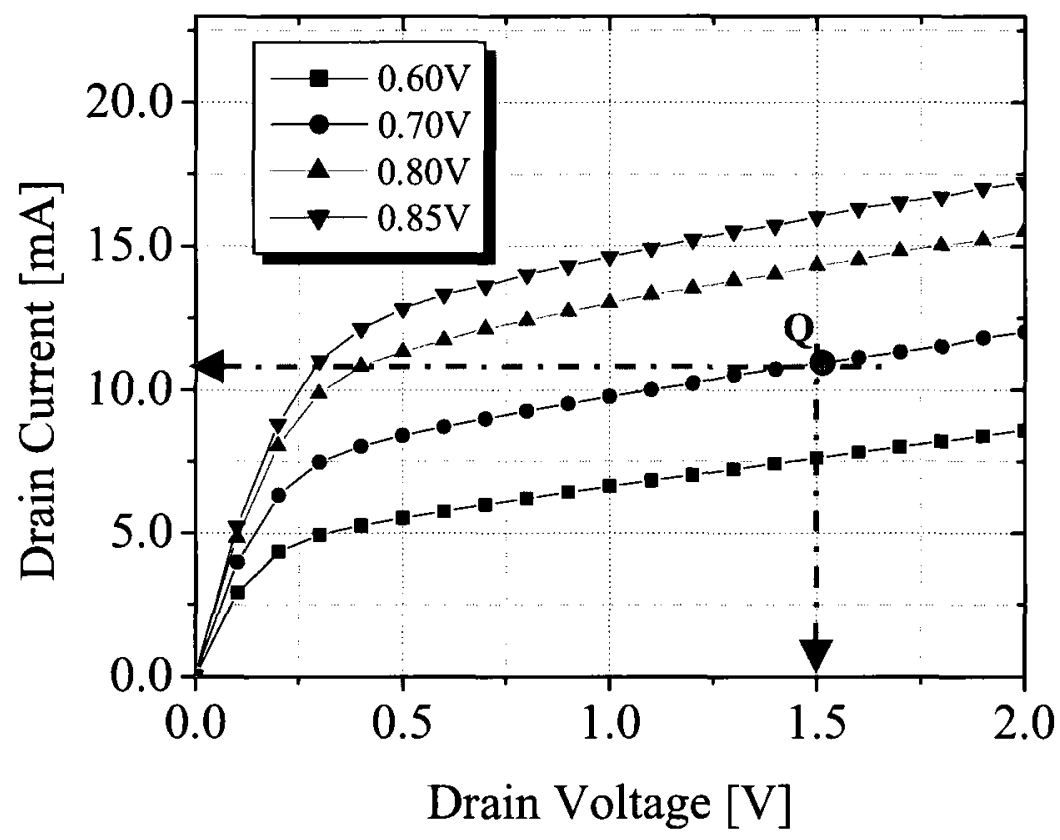

Figure 4.6: I-V Curves of the $30 \mathrm{x} 1 \mu \mathrm{m} / 0.09 \mu \mathrm{m}$ nMOS device 


\subsection{Linearity of a Low Noise Amplifier}

Linearity of a LNA is an important design consideration in receiver design. From a system-level point of view, any distortion generated in the LNA will propagate through the whole receiver chain resulting in receiver desensitization (i.e., the receiver will not be able to detect weak signals). When two or more signals that are separated in frequency are applied to the input of a low noise amplifier that is operating in its nonlinear region, the output of the amplifier will contain additional frequency components called inter-modulation products if the level of the input signals is high. To quantify the linear operation of the amplifier, it is important to determine its third-order intercept point (IP3), Figure 4.7 shows the IP3 of an amplifier.

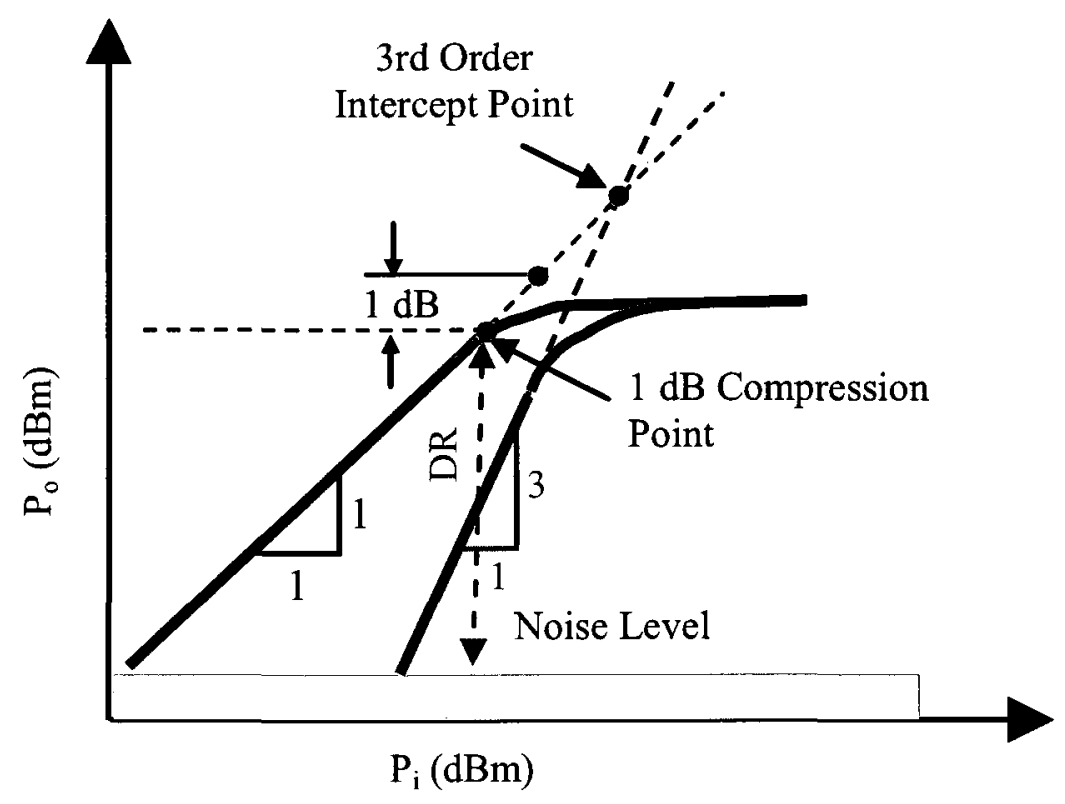

Figure 4.7: Amplifier linearity ( $\mathrm{P}_{\mathrm{IdB}}$ and IP3) 
If the input signal $V_{i n}(t)$ to an amplifier is given by:

$$
v_{i n}(t)=A\left[\cos \left(2 \pi f_{1} t\right)+\cos \left(2 \pi f_{2} t\right)\right],
$$

the output signal $v_{o}(t)$ of the amplifier can be expressed by a power series and is given by:

$$
v_{o}(t)=a_{o}+a_{1} v_{i n}(t)+a 2 v_{i n}^{2}(t)+a_{3} v_{i n}^{3}(t)+\cdots
$$

The output signal would have frequency components that include $f_{1}, f_{2}, 2 f_{1}, 2 f_{2}$, $f_{1} \pm f_{2}, 2 f_{1} \pm f_{2}, 2 f_{2} \pm f_{1}$, where $f_{1} \pm f_{2}$ are the $2^{\text {nd }}$ order inter-mod and $2 f_{1} \pm f_{2}$ and $2 f_{2} \pm f_{1}$ are the $3^{\text {rd }}$ order inter-mod. All harmonics beyond the fundamental components represent harmonic distortion $(H D)$ that is given by

$$
H D=\frac{\sqrt{\sum_{i=2}^{N} v_{i}^{2}}}{v_{1}}
$$

where $v_{\mathrm{i}}$ is the voltage of the $i^{\text {th }}$ frequency component. Figure 4.8 shows the output spectrum of the 2 nd and 3 rd order two-tone inter-modulation products.

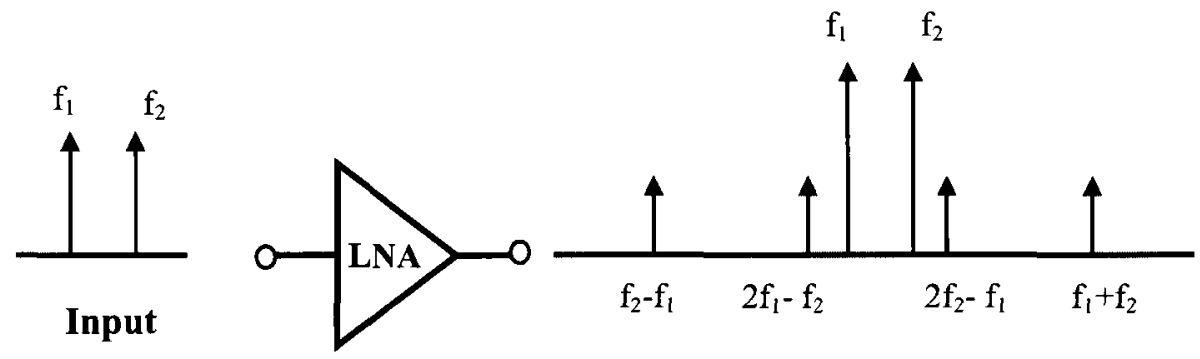

Output

Figure 4.8: Output spectrum of $2^{\text {nd }}$ and $3^{\text {rd }}$ order two-tone inter-modulation products 
In a low noise amplifier, all the second order products are undesired and they can easily be filtered from the output components because they are far from the fundamental frequency components $f_{l}$ and $f_{2}$. The $3^{\text {rd }}$ order products can not be easily filtered from the passband of the amplifier because they are close to the fundamental components. The inter-modulation products cause distortion to the output signal and such effect is called third-order inter-modulation distortion. From Figure 4.7 both the linear and third-order responses exhibit compression at high input powers. Both the linear and $3^{\text {rd }}$ order responses intersect at a hypothetical point because they have different slopes. This hypothetical point is called the $3^{\text {rd }}$ order intercept point (IP3).

The one-dB compression point $\left(P_{1 \mathrm{~dB}}\right)$ is another important design parameter because it is one of two parameters which determine the amplifier's dynamic range $(D R)$, which is given by

$$
D R=P_{1 d B}-P_{M D S}
$$

where $P_{M D S}$ is the minimum detectable signal. Therefore, higher $\mathrm{P}_{1 \mathrm{~dB}}$ would increase the dynamic range of the amplifier. For low noise amplifiers, the operation is limited by the noise level and the maximum power level for which inter-modulation distortion becomes unacceptable. This operating range is called spurious-free dynamic range and is given by

$$
S F D R=\frac{2}{3}\left(I P 3-P_{M D S}\right)
$$




\subsection{Design of a V-band LNA with LG-CPW Transmission Lines}

The design of the amplifier presented here is a new approach to CMOS integrated circuits design; this design utilizes lower-ground CPW transmission lines as matching elements to overcome the CMOS design rules limitations. These limitations could prevent the realization of low and high impedances that might be needed for the amplifier's matching networks, to meet the design objectives of having optimal performance. The amplifier of this study used a cascode topology which features highgain and good isolation between the input and the output of the amplifier. Figure 4.9 shows a core cascode amplifier which consists of a common-source device followed by a common gate device. The common-source device (Q1) that is used in this work is an nMOS transistor with 30 fingers and finger width of $1 \mu \mathrm{m}$, and the common-gate device (Q2) is an nMOS transistor with 32 fingers and finger width of $1.25 \mu \mathrm{m}$. Both Q1 and Q2 are sized to ensure the core cascode amplifier is optimized for minimum noise figure and the best achievable gain at this minimum $N F$. The test bench for characterizing the core cascode amplifier is shown in Figure 4.10.

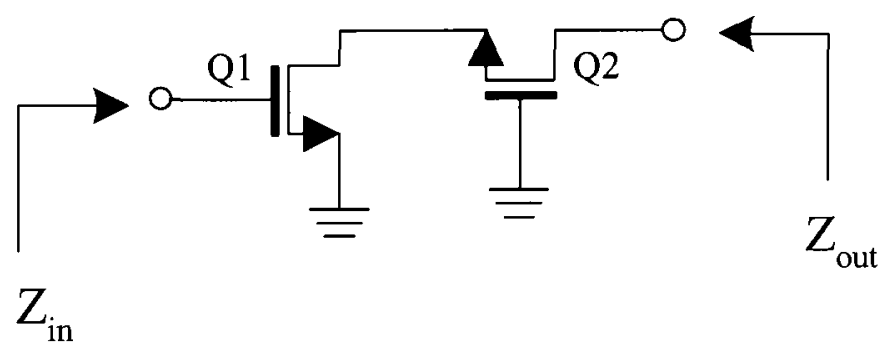

Figure 4.9: Cascode Amplifier Configuration 
The proposed single-stage amplifier is implemented in a 90-nm CMOS process which has 9-metal layers. The main design specifications of this single-stage amplifier include a noise figure less than $6 \mathrm{~dB}$ and gain of at least $10 \mathrm{~dB}$ at $57 \mathrm{GHz}$ operating frequency, and a third-order intercept point greater than $0 \mathrm{dBm}$. Figure 4.11 shows the simulated $N F_{\min }$ and the $G_{\max }$ of the core cascode amplifier without matching networks.

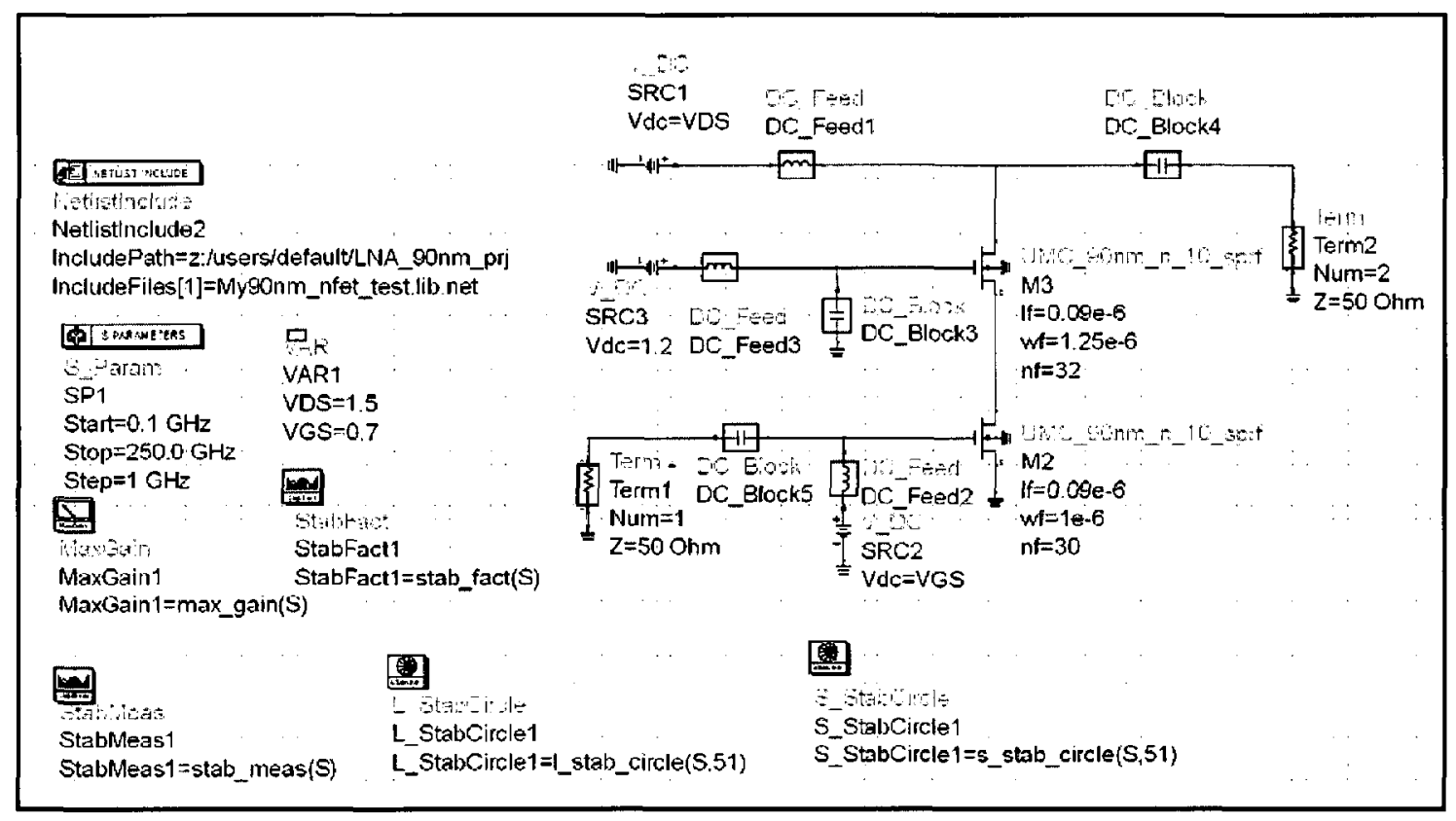

Figure 4.10: Test bench for simulating the core cascode amplifier 


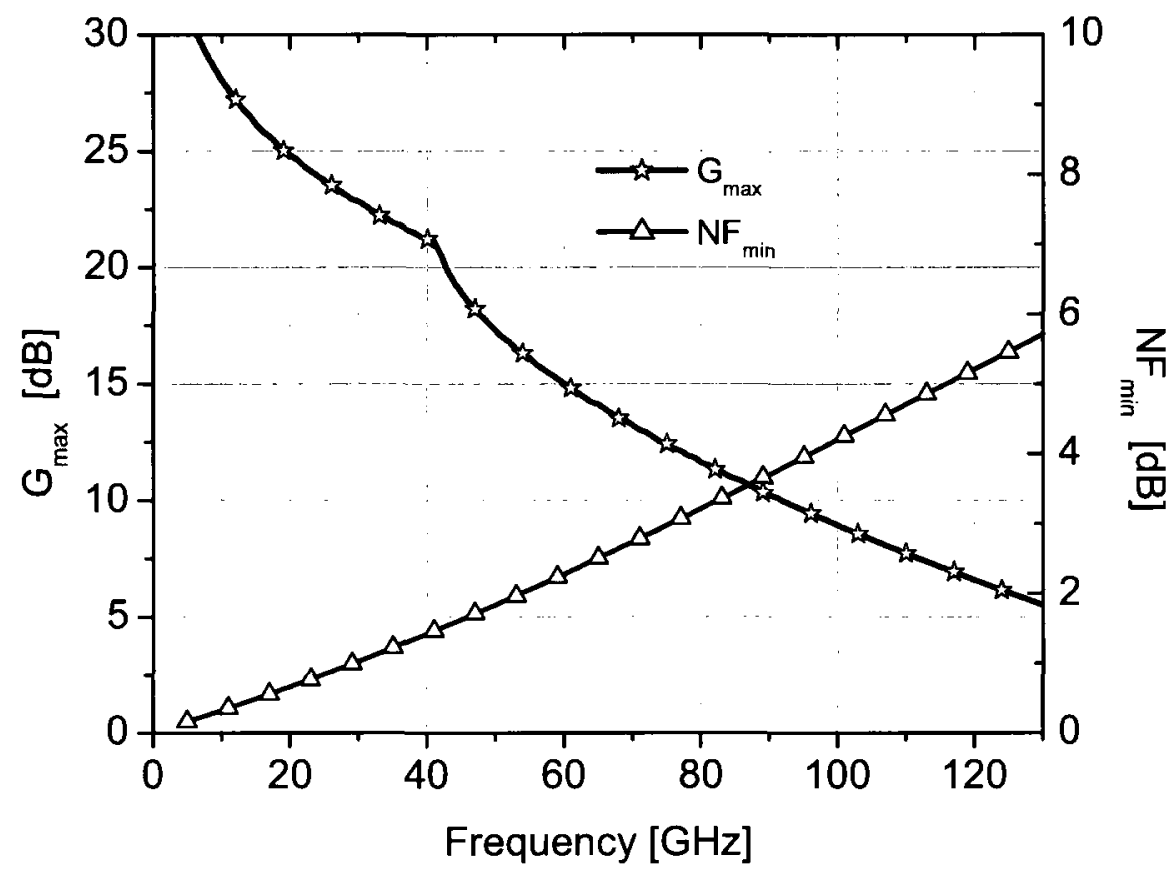

Figure 4.11: Simulated $\mathrm{NF}_{\min }$ and $\mathrm{G}_{\max }$ of the core cascode w/o matching (Q1 is $30 \times 1 / 0.09, \mathrm{Q} 2$ is $32 \times 1.25 / 0.09, \mathrm{~V}_{\mathrm{GS}}=0.7 \mathrm{~V}, \mathrm{~V}_{\mathrm{DS}}=1.5 \mathrm{~V}$ )

From the results of Figure 4.11, this core cascode without matching can provide $G_{\max }$ greater than $15.5 \mathrm{~dB}$ and $N F_{\text {min }}$ of less than $2.2 \mathrm{~dB}$ at the operating frequency of interest of $57 \mathrm{GHz}$. The optimal reflection coefficient $\left(\Gamma_{\text {opt }}\right)$ of the core cascode is obtained form the circuit simulator (Agilent Advanced Design System, ADS) and is matched to the source impedance to achieve minimum noise figure. As an initial step in the amplifier's design, the matching networks are designed with ideal transmission lines to determine the characteristic impedances and electrical lengths of the matching elements. The input matching network is optimized for minimum noise figure whereas the output matching is designed to provide the best achievable gain while maintaining low noise figure. Figure 4.12 illustrates the conditions that are required to obtain the best achievable gain while maintaining optimal noise figure. 


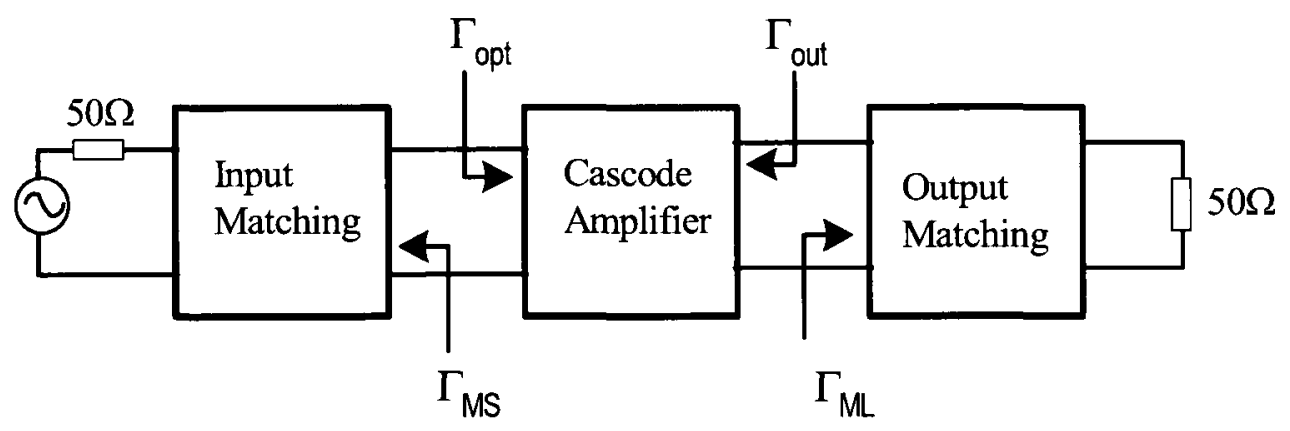

Figure 4.12: Representation of the output conjugate match for optimal noise performance $\left(\Gamma_{\text {out }}\right)^{*}=\Gamma_{\mathrm{ML}}, \Gamma_{\mathrm{MS}}=\Gamma_{\mathrm{opt}}$

In Figure 4.12, $\Gamma_{\mathrm{ML}}$ is the reflection coefficient of the load matching network and $\Gamma_{\mathrm{MS}}$ is the reflection coefficient of the source matching network. $\Gamma_{\mathrm{ML}}$ is given by

$$
\Gamma_{M L}=\Gamma_{o u t}^{*}=\left(S_{22}+\frac{S_{12} S_{21} \Gamma_{o p t}}{1-S_{11} \Gamma_{o p t}}\right)^{*}
$$

Figure 4.13 shows the schematic diagram of the single stage-amplifier which is designed with ideal transmission lines matching elements. 


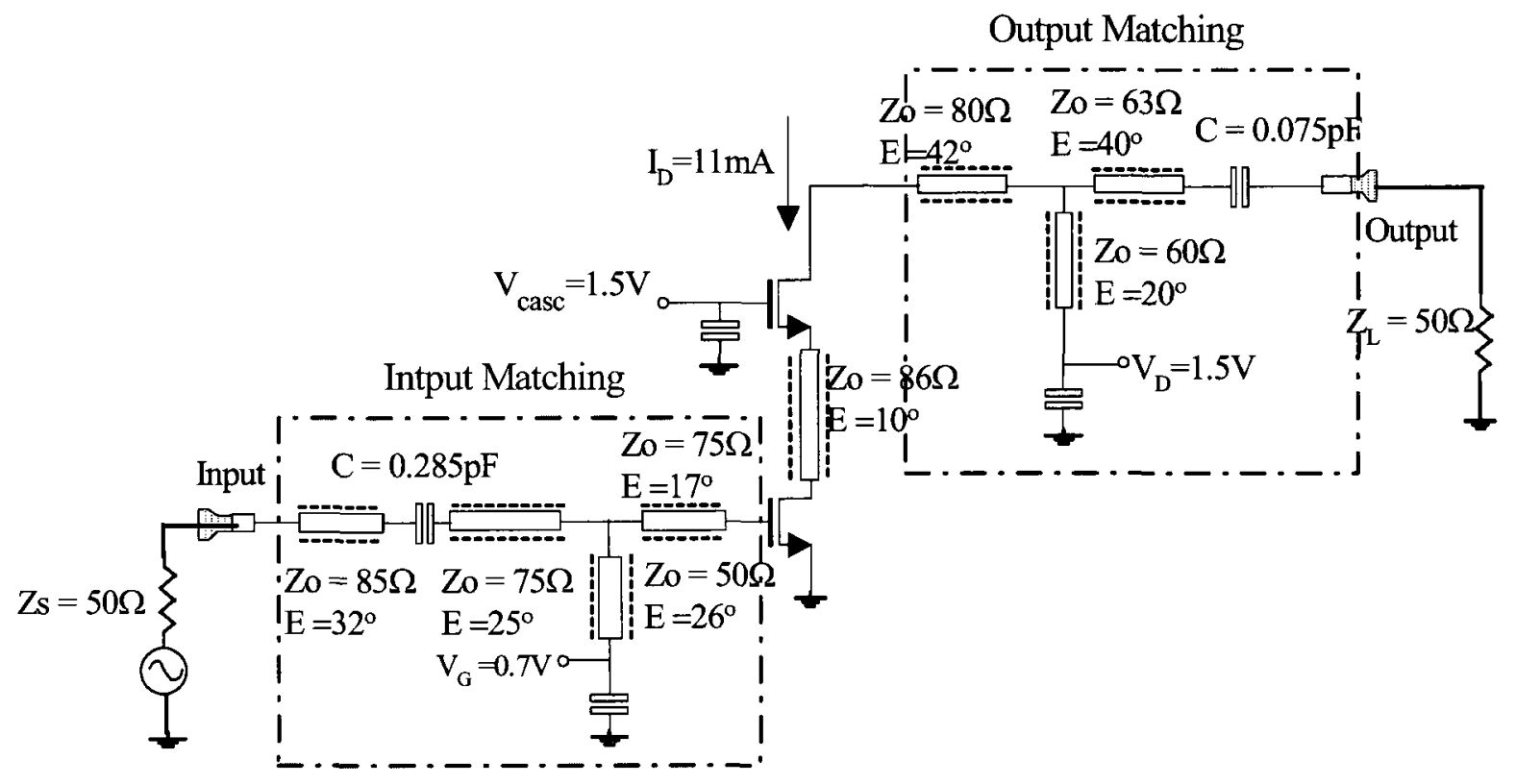

Figure 4.13: Circuit schematic of a V-band single-stage amplifier with ideal transmission line matching-elements.

Knowing the electrical lengths and characteristic impedances of the matching elements, the next step is to replace the ideal transmission lines by their equivalent physical layout of LG-CPW elements (i.e., same characteristic impedances and electrical lengths). The simulated noise figure and gain of the amplifier of Figure 4.13 are presented in Figure 4.14. From Figure 4.14, this transmission-line-based single-stage amplifier can provide gain greater than $10 \mathrm{~dB}$ and a noise figure of less than $4 \mathrm{~dB}$ over a frequency range of 54-69 GHz. However, the ideal transmission lines do not take into account the insertion loss of the matching elements. Therefore, it is essential to perform post-layout simulation (i.e., simulation of the physical layout) to have results that are a true representation of the amplifier to be developed. 


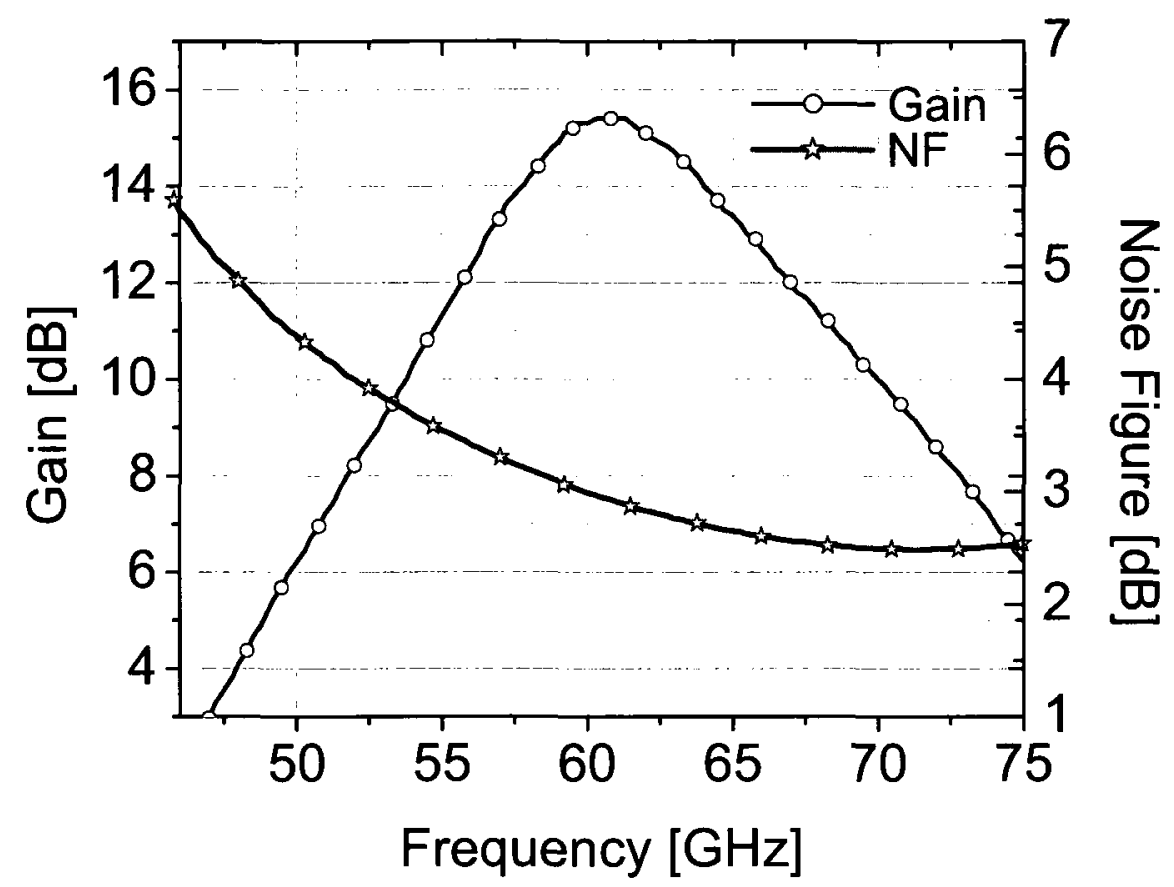

Figure 4.14: Simulated NF and gain of the single-stage amplifier of ideal TL matchingelements

The simulated input and output return losses of the single-stage amplifier shown in Figure 4.13 are presented in Figure 4.15. From Figure 4.15, the input return loss is -10 $\mathrm{dB}$ at the intended operating frequency of $57 \mathrm{GHz}$, and the output return loss is $-7 \mathrm{~dB}$. Since the use of ideal transmission lines is an initial step in the amplifier design, further optimization of the amplifier's performance is better to be performed in the post-layout simulation where the ideal elements are replaced by their physical layout. 


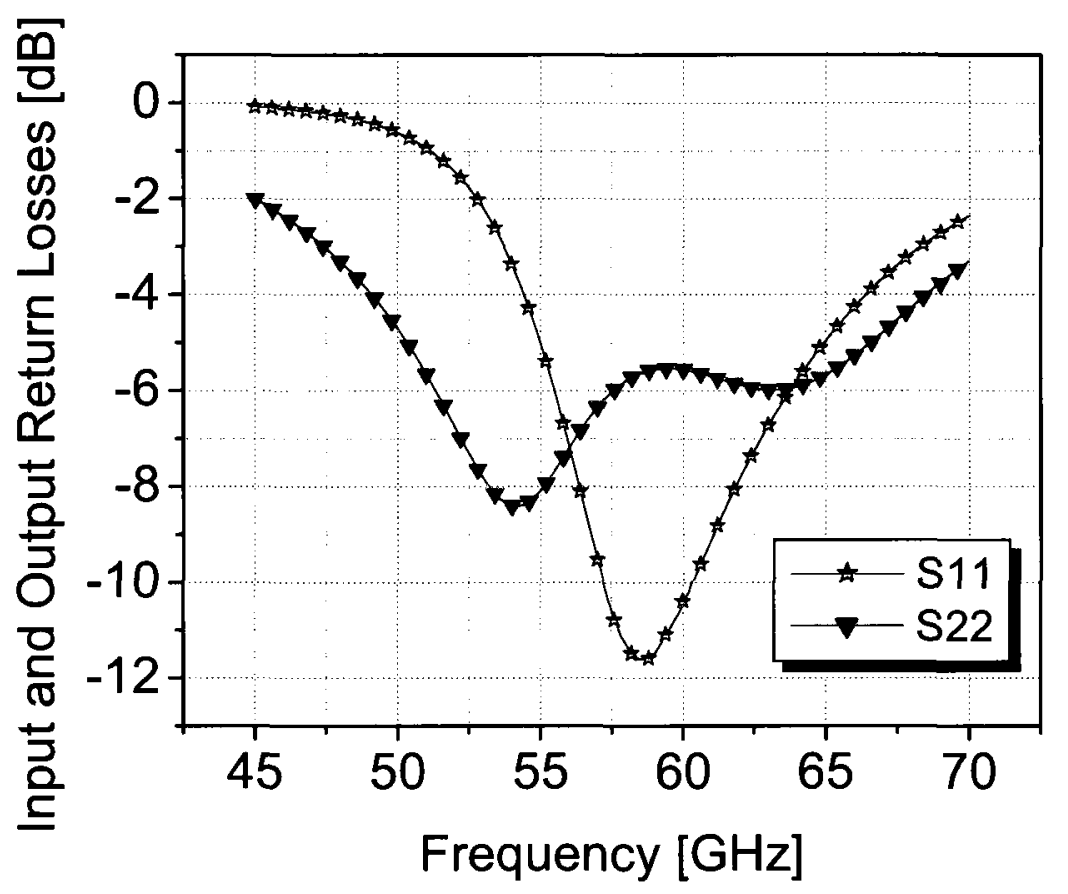

Figure 4.15: Simulated input and output return losses of the single-stage amplifier

In addition to having low $N F$, high gain, low power consumption, and high linearity, the amplifier must provide unconditionally stable performance. For an unconditionally stable operation, the stability factor ( $k$ ) (also called Rollet's factor) should be greater than 1 , and the stability measurement $(B)$ should be greater than zero [30], $k$ and $B$ are given by

$$
k=\frac{1-\left|S_{11}\right|^{2}-\left|S_{22}\right|^{2}+|\Delta|^{2}}{2\left|S_{12} S_{21}\right|}>1,
$$

where

$$
\begin{aligned}
& \Delta=\left|S_{11} S_{22}-S_{12} S_{21}\right|<1, \\
& B=1+\left|S_{11}\right|^{2}-\left|S_{22}\right|^{2}-|\Delta|^{2}>0,
\end{aligned}
$$


Figure 4.16 shows a flow-chart which summarizes the design flow.

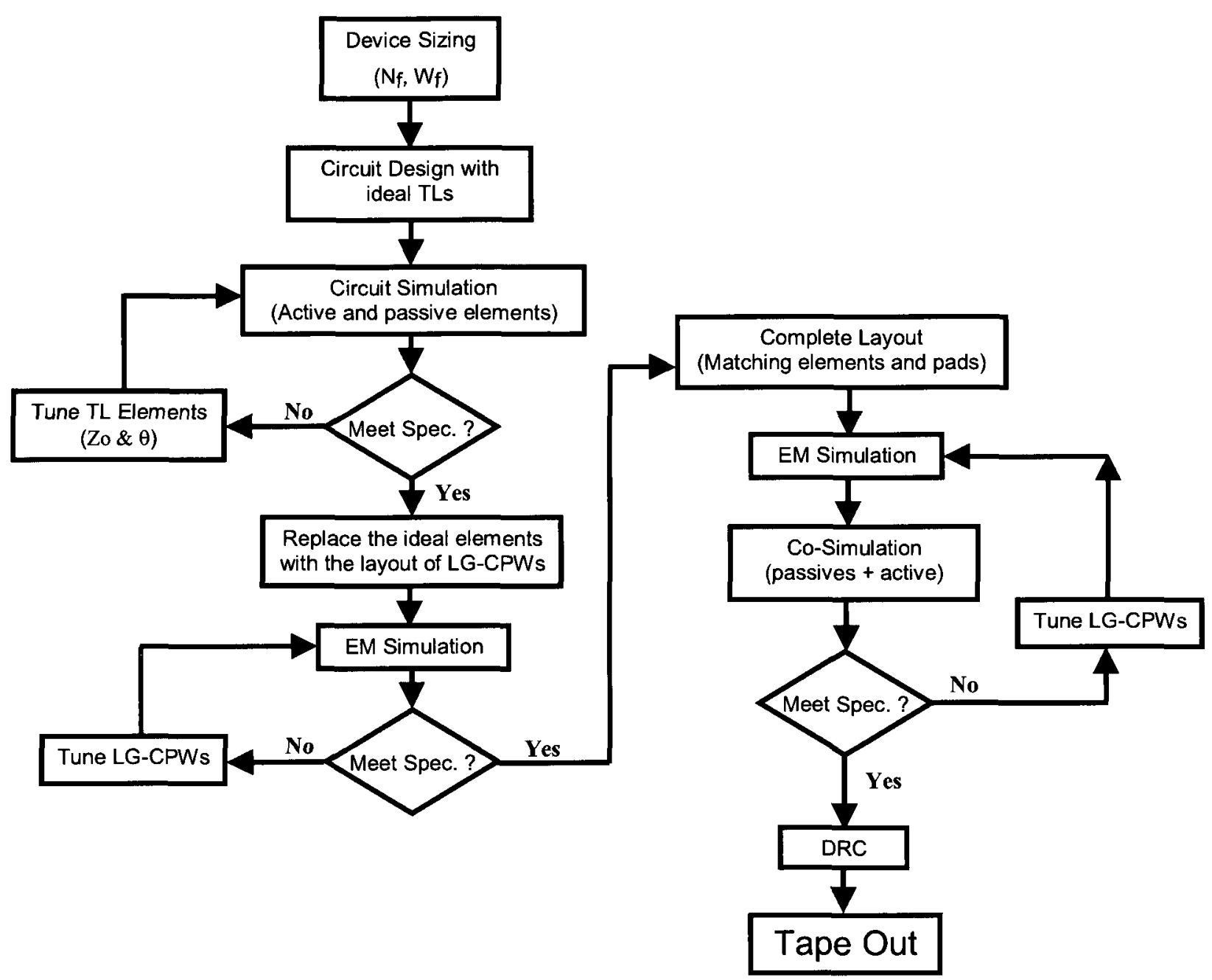

Figure 4.16: Design flow of a LG-CPW Low Noise Amplifier 


\subsection{Layout of the Amplifier's Matching Networks}

From the circuit schematic of the single-stage amplifier shown in Figure 4.13, the input matching network consists of series transmission lines with characteristic impedances of 75 and $85 \mathrm{ohms}$, a short circuited stub with characteristic impedance of $50 \mathrm{ohms}$, and a series capacitor of $0.285 \mathrm{pF}$. The physical layout of these matching elements is realized with LG-CPW transmission lines that have the same characteristic impedances and electrical lengths as the ideal transmission line elements. The post-layout simulation of the input matching network is performed using an electromagnetic (EM) simulator (Momentum) to take into account the electromagnetic coupling between the matching elements. Similarly, the physical layout of the output matching network was simulated using an EM simulator. Figures 4.17 show the 3-D view of the physical layout of the input and output matching networks. The S-parameters of the matching networks are extracted from the EM simulator and then used as two-port S-parameter blocks in a circuit simulator (ADS) to characterize the amplifier's performance. The series capacitors and the dc blocking capacitors are realized using the metal layers and the $\mathrm{SiO}_{2}$ of the CMOS substrate, to form MIM capacitors. 


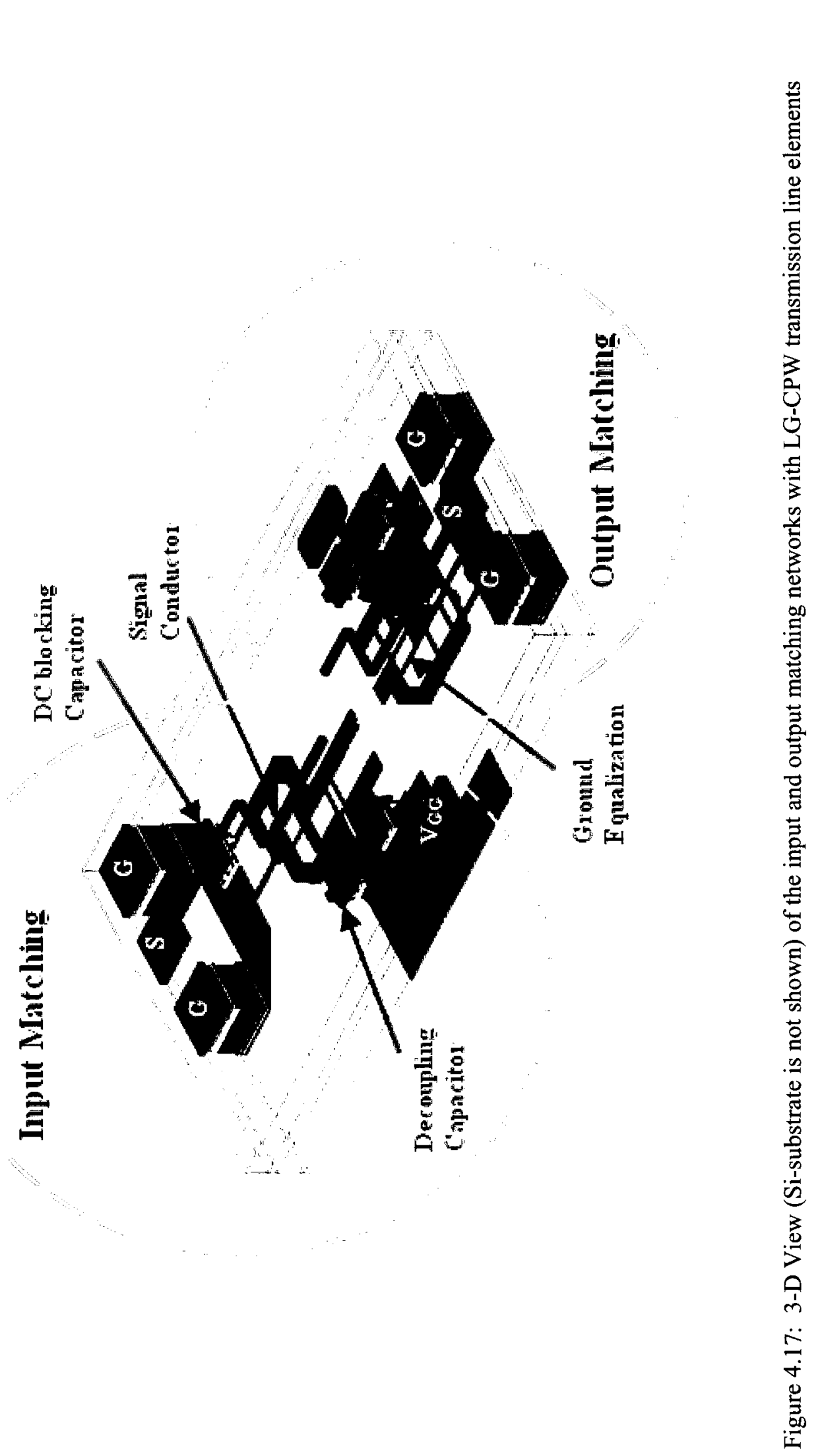




\subsection{Experimental and Post-layout Simulation Results}

The on-wafer noise figure measurements of the amplifier chip, shown in Figure 4.18, were performed using an Agilent noise figure analyzer (N8975A) with three downconverters (N8975A-K50, N8975A-K63, N8975A-K75), and two noise sources (346C-K01, NC5115). Figure 4.19 shows a block diagram of the noise figure test setup. The noise figure measurement were performed at the test facilities of the Chip Implementation Center (CIC) in Taiwan. Figure 4.20 shows the test environment at CIC.

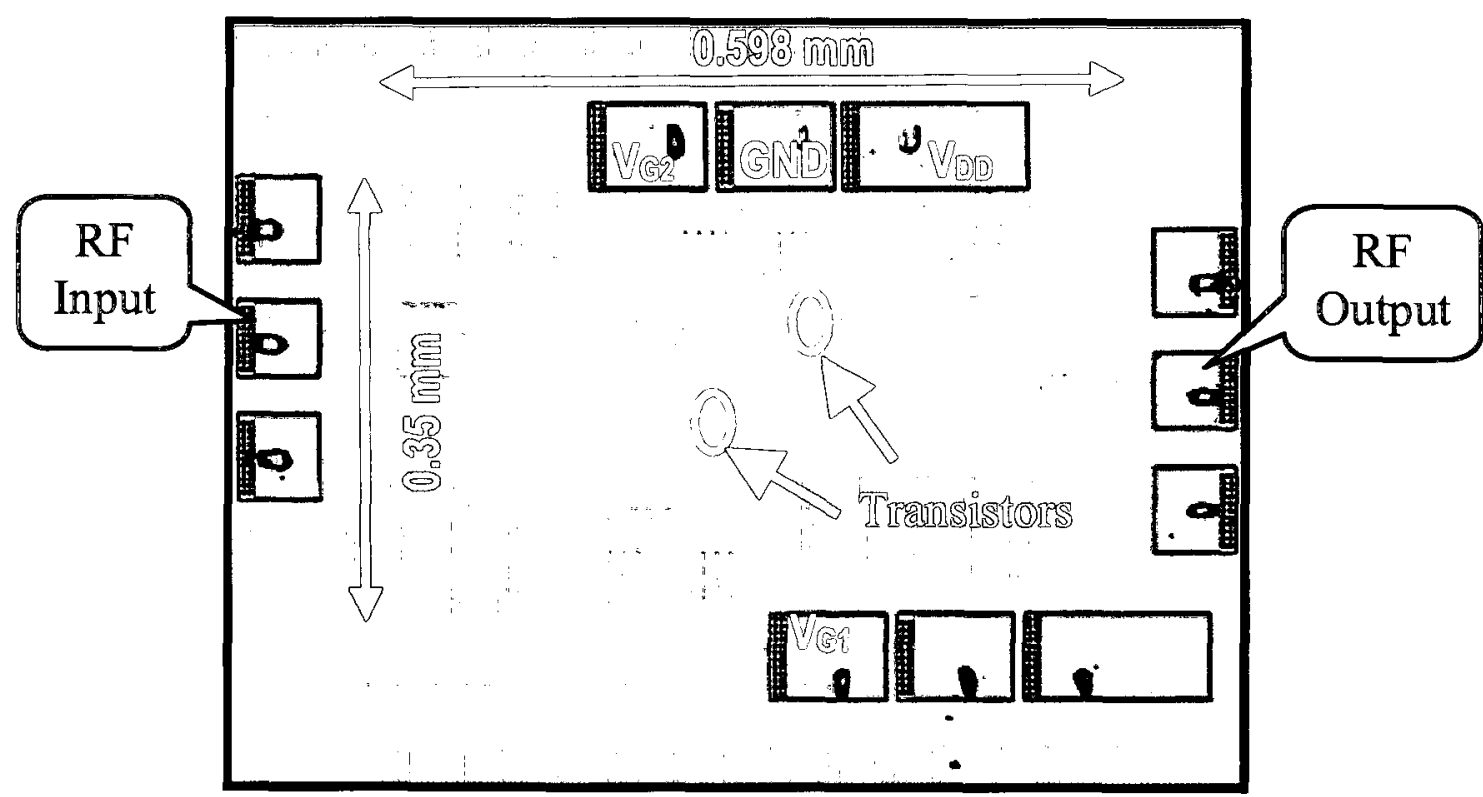

Figure 4.18: Photograph of the 90-nm CMOS $57 \mathrm{GHz}$ single-stage amplifier of this work (chip area is $0.2 \mathrm{~mm} 2$ ) 
Agilent Noise Figure Analyzer

(N8975A)

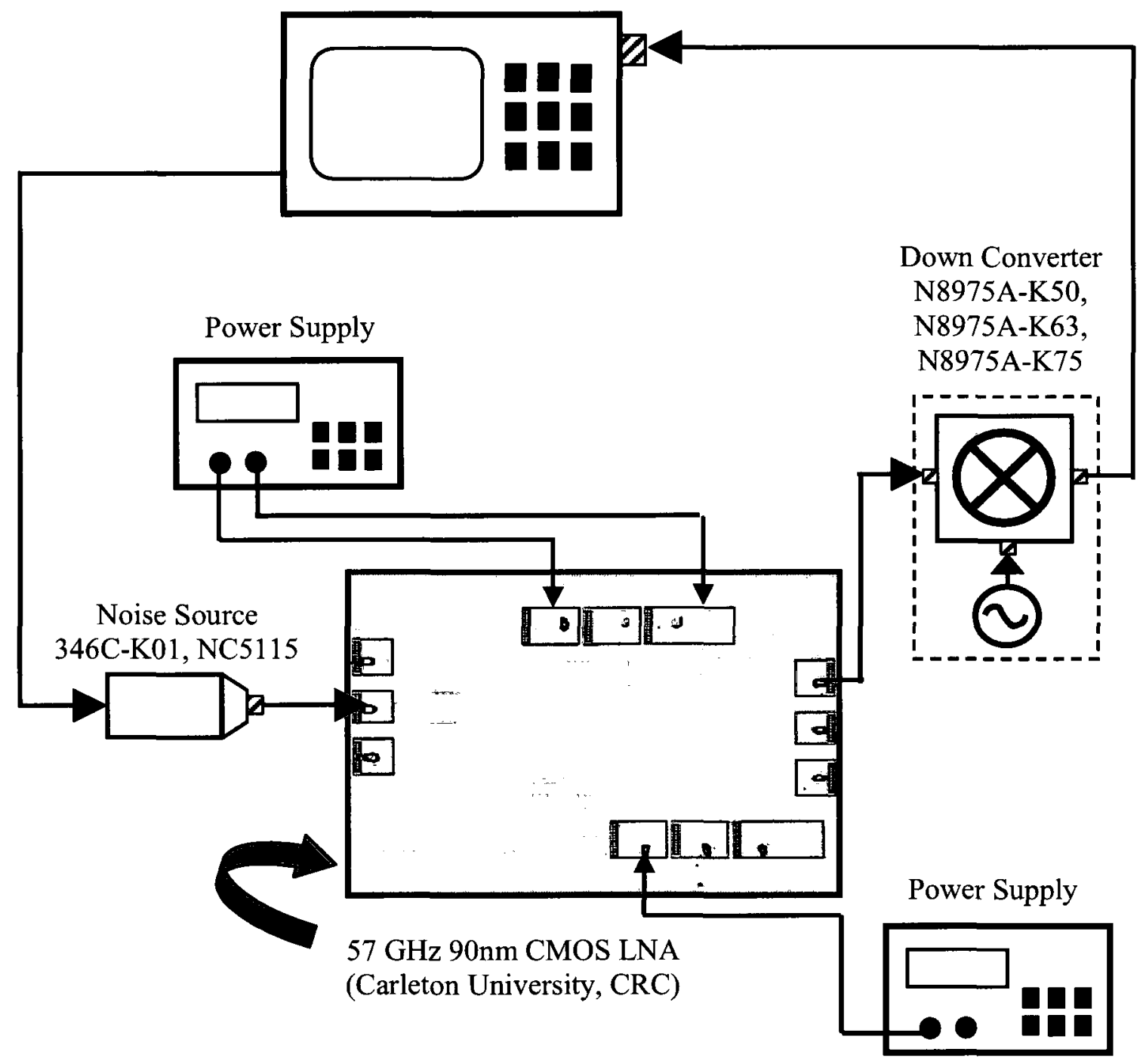

Figure 4.19: Block diagram of the noise figure test setup for characterizing the singlestage LG-CPW LNA. 


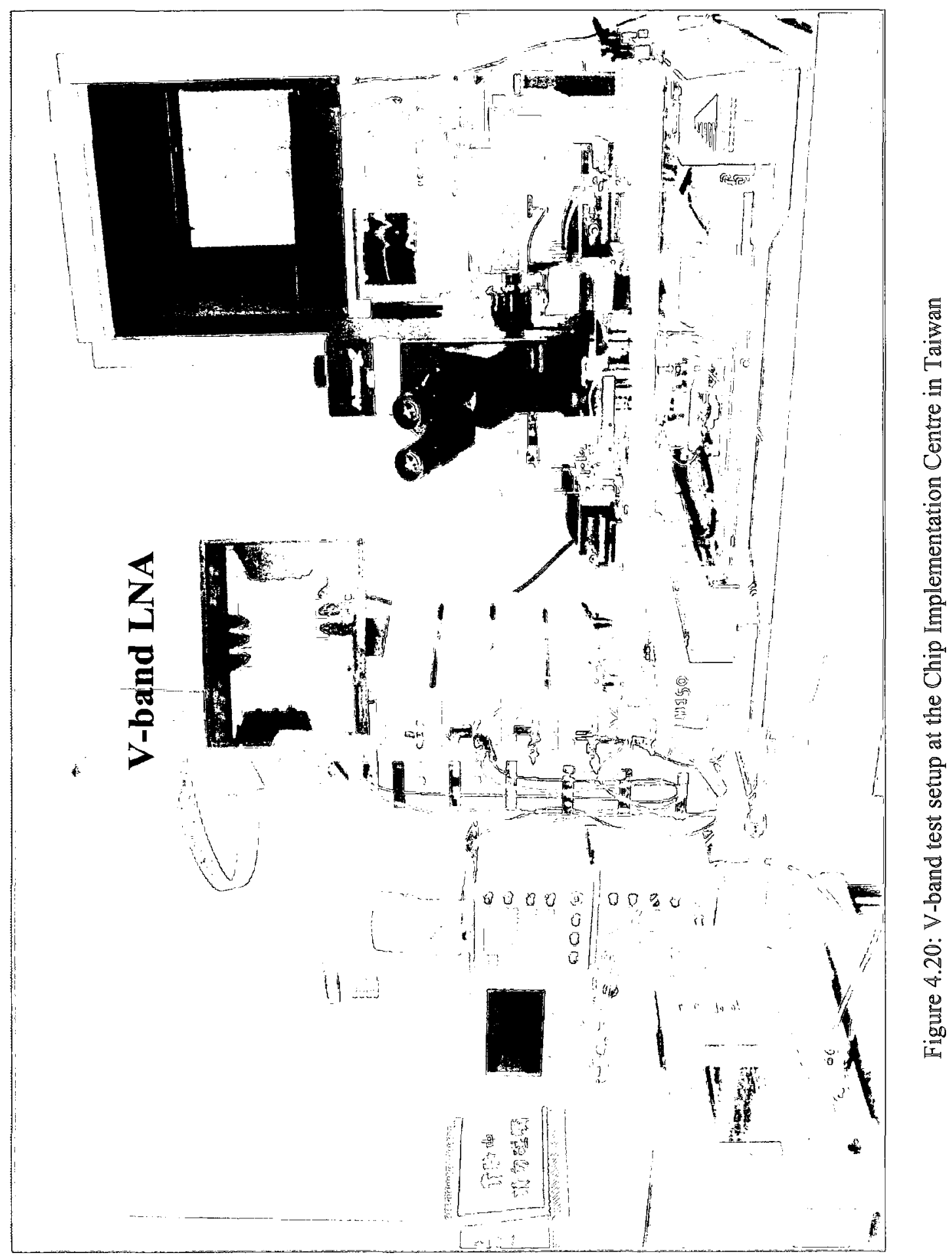


The measured and post-layout simulation results of the noise figure of the single-stage amplifier are shown in Figure 4.21. The results of Figure 4.21 demonstrate that the measured NF is less than $4.1 \mathrm{~dB}$ in the frequency range of $57-60 \mathrm{GHz}$, which is better than the simulation results. The discrepancy between the measurements and simulation is attributed to inaccurate transistor's model at $\mathrm{mmW}$ frequencies.

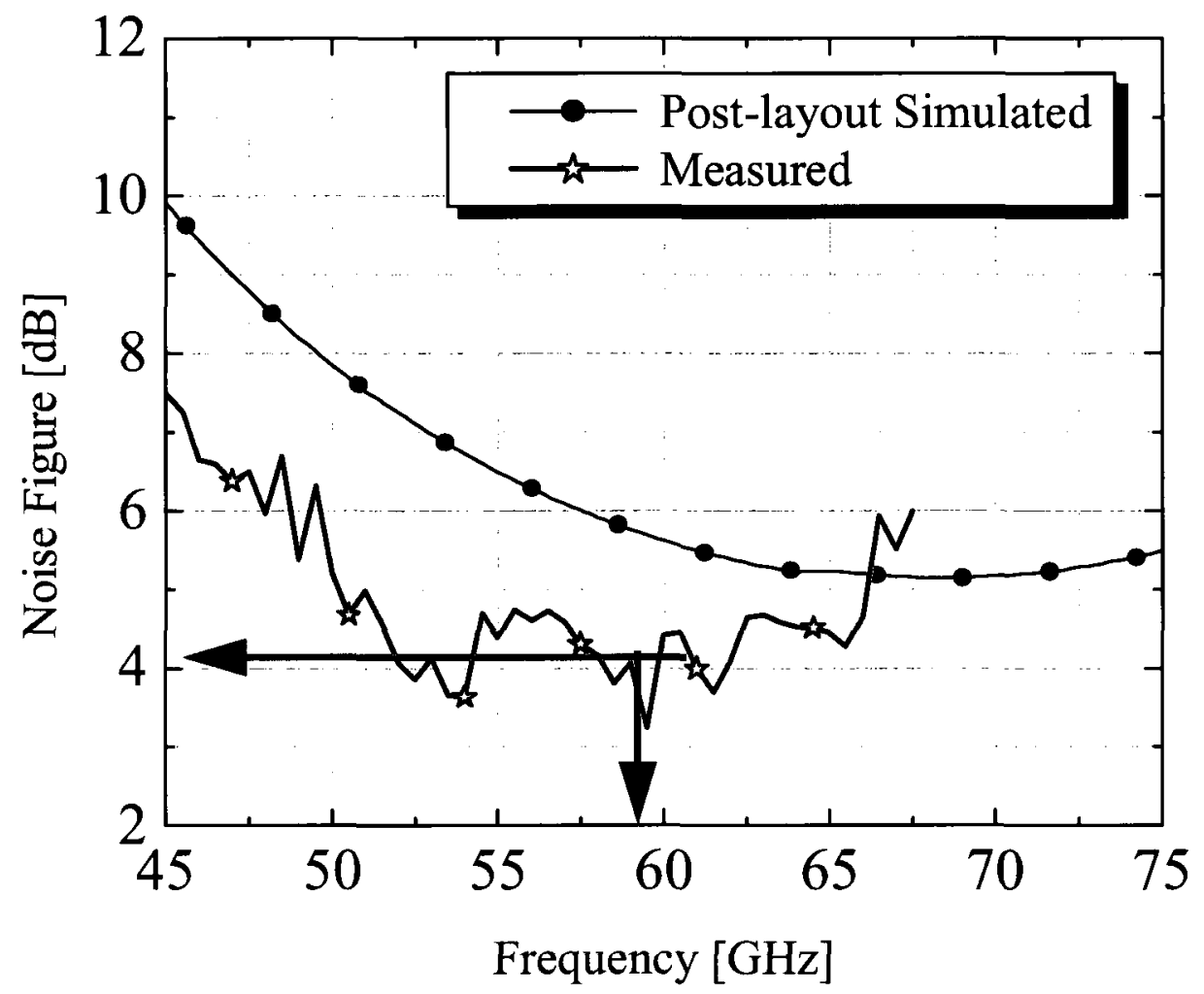

Figure 4.21: Measured and post-layout simulated NF of the CMOS LG-CPW singlestage amplifier $(\mathrm{Vdd}=1.5 \mathrm{~V}, \mathrm{Idd}=11 \mathrm{~mA})$ 
The gain and the return losses of the amplifier were measured using a $67 \mathrm{GHz}$ vector network analyzer (Agilent E8361A). Figure 4.22 shows the measured and post-layout simulated gain.

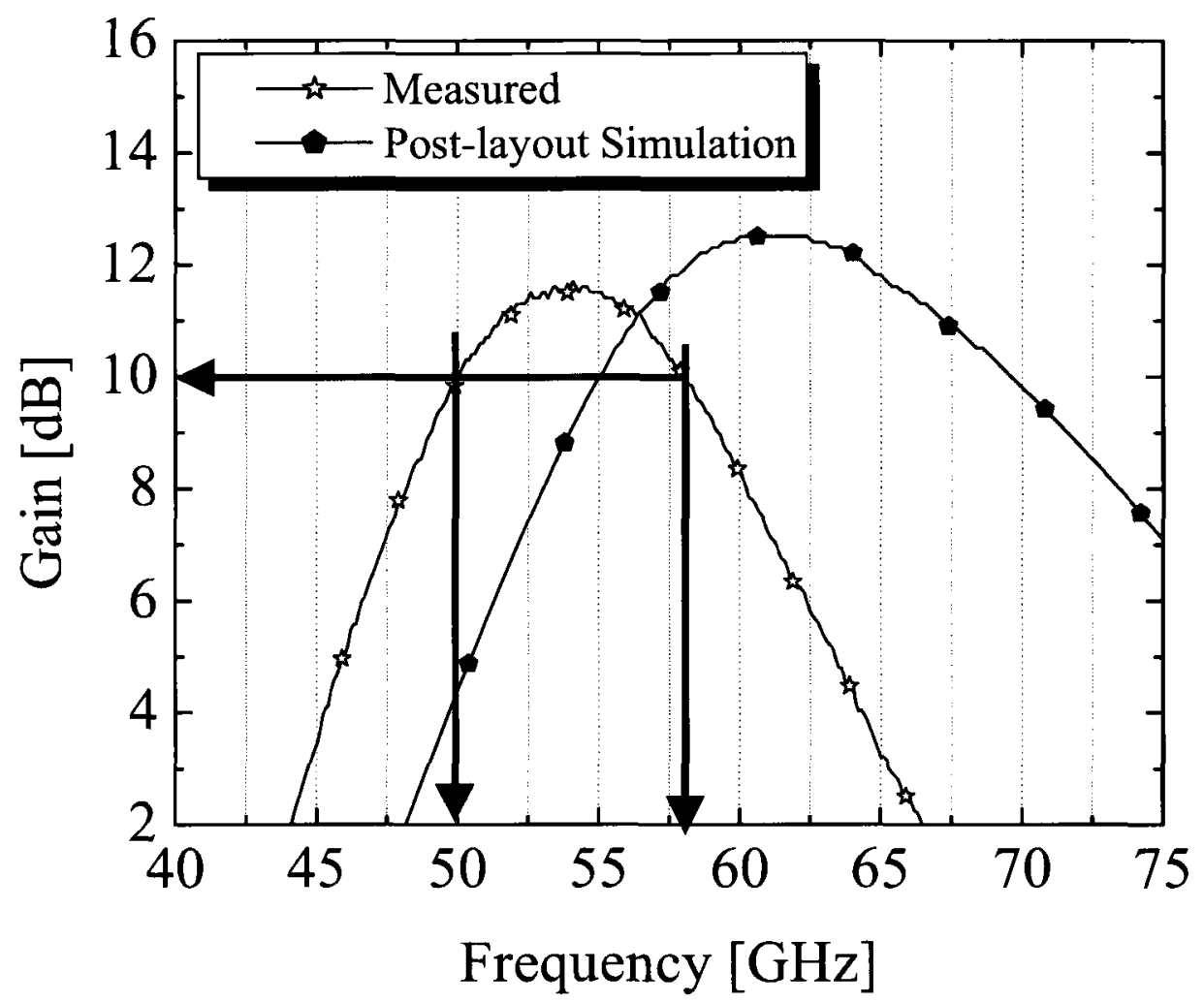

Figure 4.22: Measured and post-layout simulated gain of the CMOS EC-CPW singlestage amplifier $(\mathrm{Vdd}=1.5 \mathrm{~V}, \mathrm{Idd}=11 \mathrm{~mA})$

From Figure 4.22, the measured gain is better than $10 \mathrm{~dB}$ in the frequency range of 50$58 \mathrm{GHz}$. The measurements of the gain showed a down shift in the frequency response in comparison with the post-layout simulation results. However, the measured gain still meets the design specification of $10 \mathrm{~dB}$ of gain at $57 \mathrm{GHz}$. Such a shift in the frequency is attributed to the accuracy of the transistor's model. Figure 4.23 shows the 
measured and post-layout simulated input return losses. From Figure 4.23, the measurements are better than $-12.5 \mathrm{~dB}$ over a frequency range of $55-61 \mathrm{GHz}$. The results of Figure 4.23 reveal that the measurements and simulation results closely agree at $57 \mathrm{GHz}$. The measured and post-layout simulated output return losses are shown in Figure 4.24.

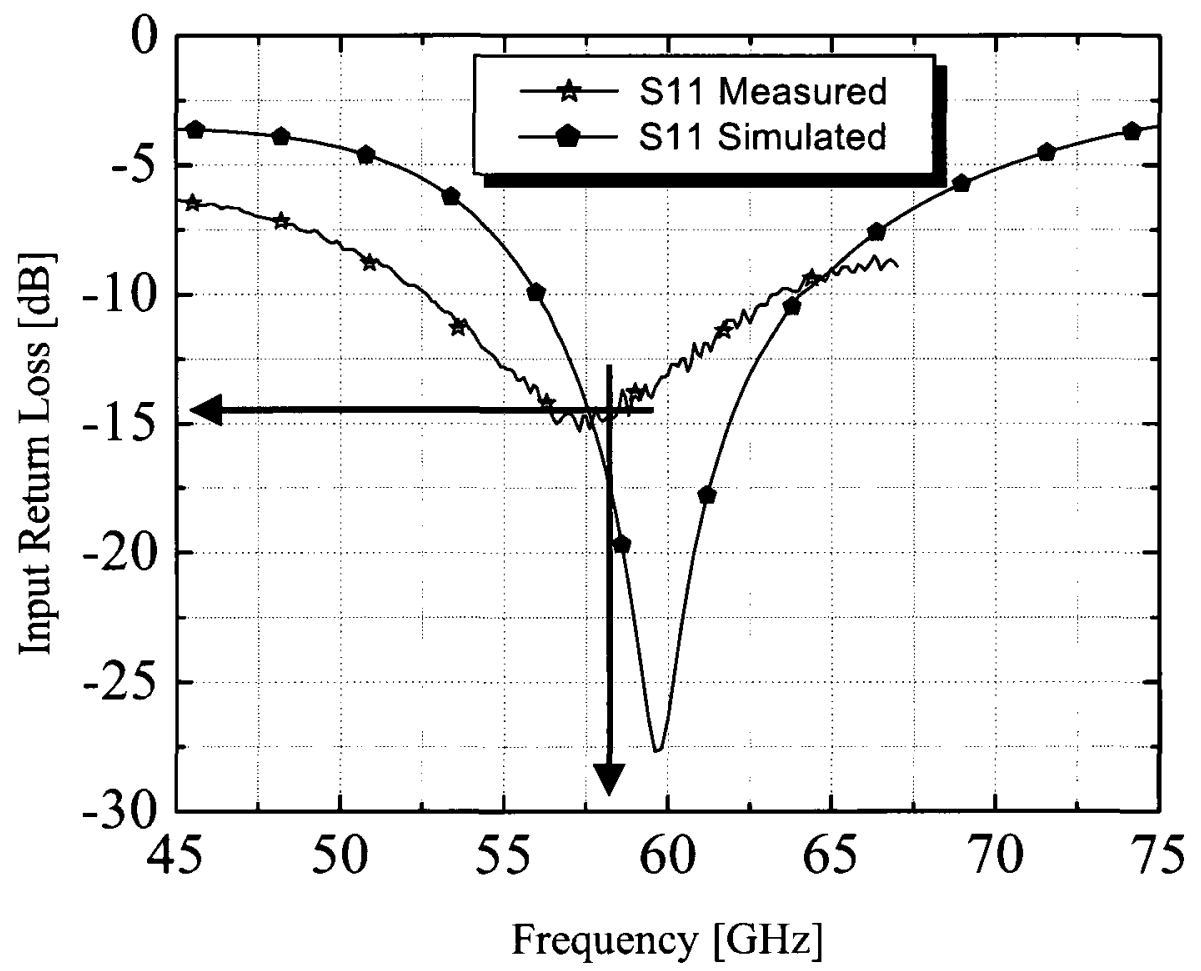

Figure 4.23: Measured and post-layout simulated input return losses of the CMOS LG$\mathrm{CPW}$ single-stage amplifier $(\mathrm{Vdd}=1.5 \mathrm{~V}, \mathrm{Idd}=11 \mathrm{~mA})$ 


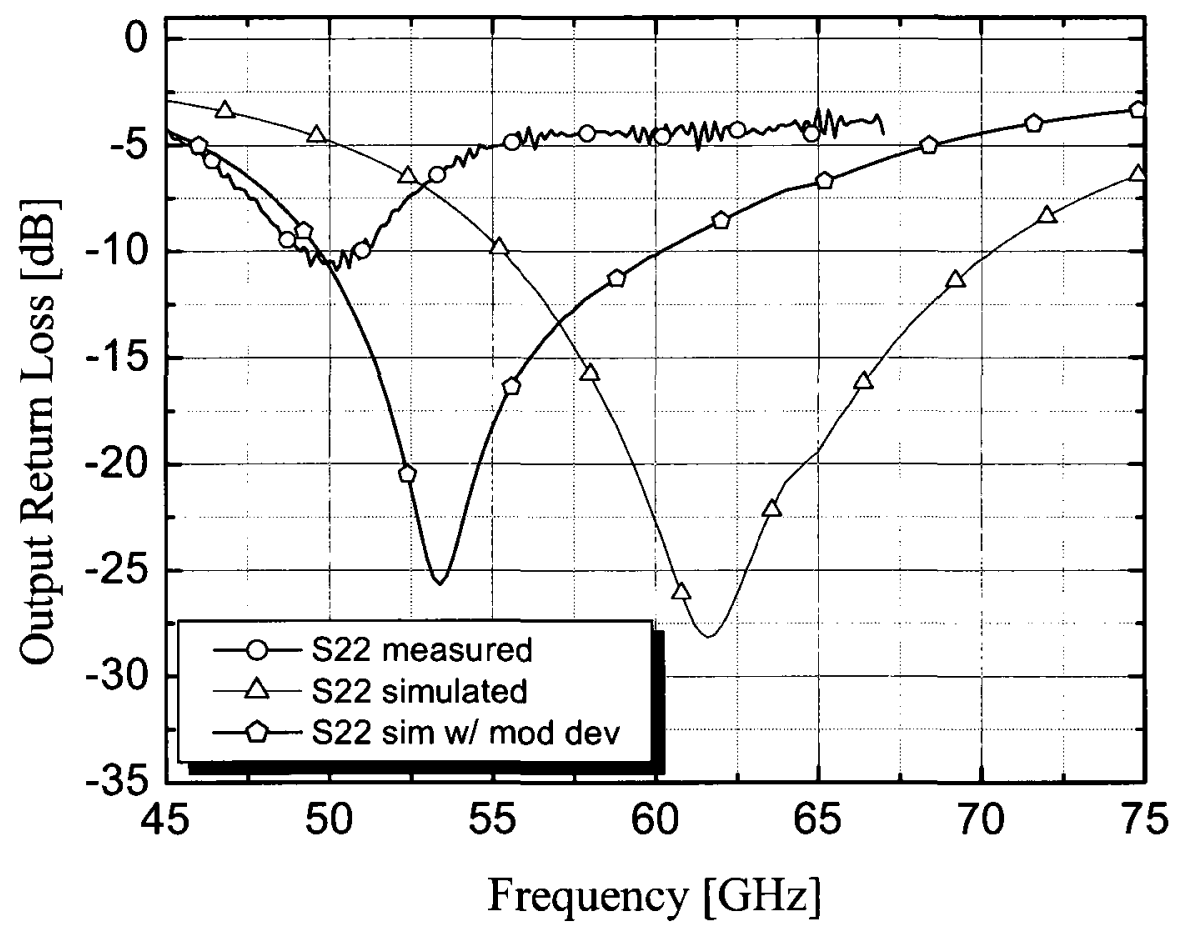

Figure 4.24: Measured and post-layout simulated S22, and simulated S22 with modified transistor model of the CMOS LG-CPW single-stage amplifier

From Figure 4.24, the response of the measured output return loss is shifted down in frequency, which resulted in a return loss higher than that of the simulated return loss at $57 \mathrm{GHz}$. By tuning the value of the $C_{\mathrm{ds}}$ of the device model, the simulated return loss moved closer to the measured results as shown in Figure 4.24. This indicates that the device model of the CMOS design kit which was used here needs to be validated at frequencies beyond $30 \mathrm{GHz}$ (the design kit is only validated up to $30 \mathrm{GHz}$ only). The test setup for measuring the gain and return losses of the single-stage chip is shown in Figure 4.25. 


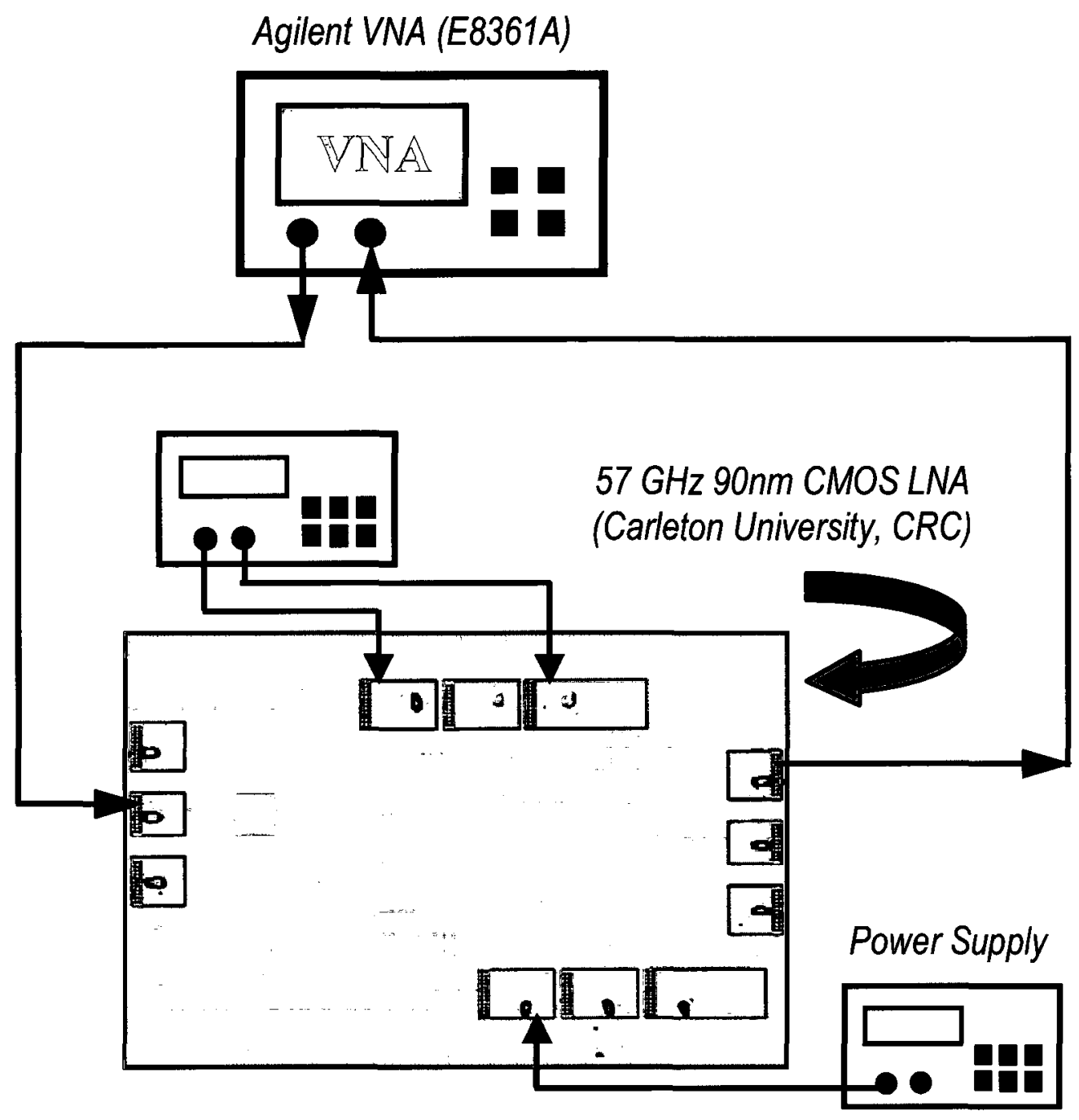

Figure 4.25: Test setup for measuring the gain and return losses of the single-stage LNA chip.

The linearity measurement of the amplifier demonstrated an input IP3 of $3 \mathrm{dBm}$ at 57 GHz with a $100 \mathrm{MHz}$ tone space, Figure 4.26 shows the measured IP3. The measured IP3 is higher than other recently published results on IP3 for V-band 90nm CMOS 
amplifiers [69]. Figure 4.27 shows the test setup which is used to measure the amplifier's IP3.

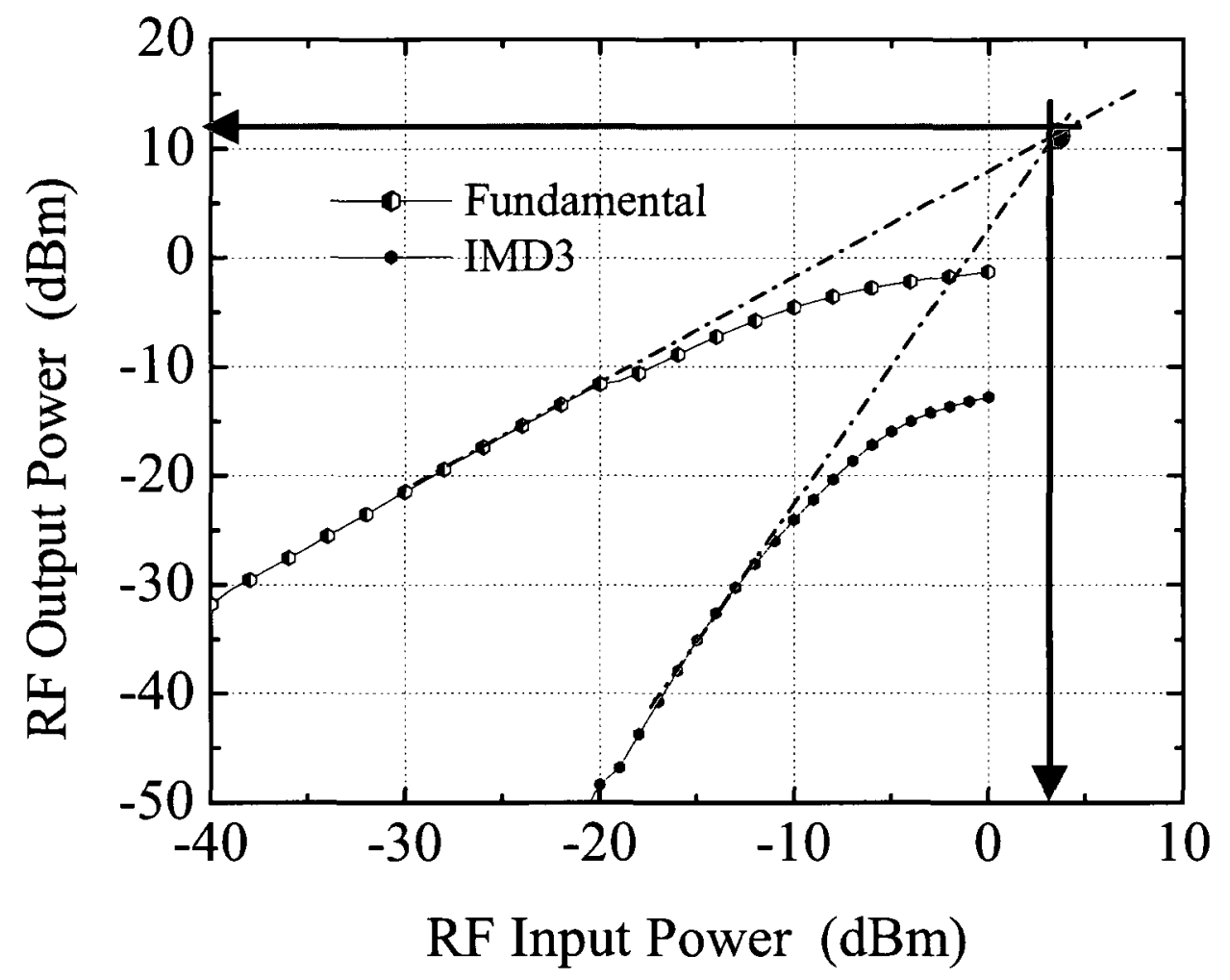

Figure 4.26: Measured $\mathrm{IP}_{3}$ of a $57 \mathrm{GHz}$ EC-CPW Single-Stage Amplifier with 100 $\mathrm{MHz}$ Tone Spacing 
Spectrum Analyzer

Agilent E4448A (3 Hz - $50 \mathrm{GHz})$

$+$

Preselected Millimeter Mixer Agilent $11974 \mathrm{~V}(50 \mathrm{GHz}-75 \mathrm{GHz})$

Signal Generator

Agilent E8257D

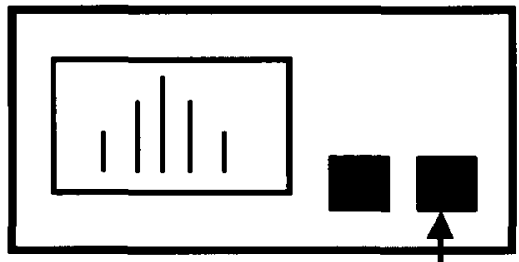

$(250 \mathrm{KHz}-67 \mathrm{GHz})$

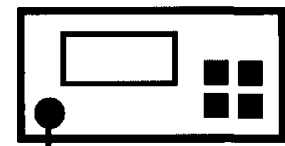

$57 \mathrm{GHz}$ 90nm CMOS LNA

(Carleton University, CRC)
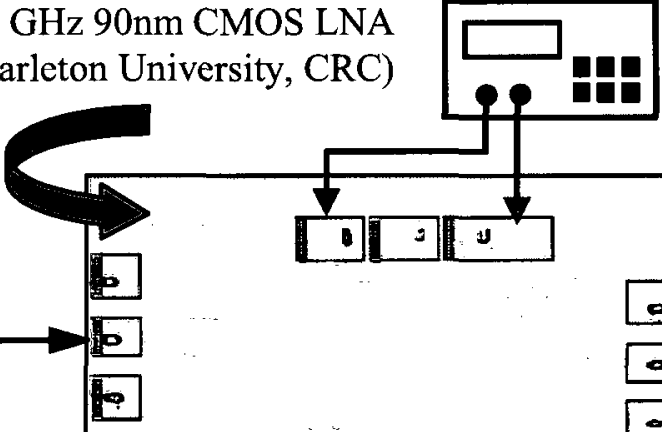

Matched Hybrid Tee

Quinstar

(QJH-ULFB00)

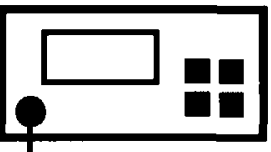

Signal Generator

Agilent E8257D

(250 KHz- 67GHz)

Power Supply

Figure 4.27: Test set up for measuring the third-intercept point of the single-stage amplifier 
A summary of the measurements of this single-stage amplifier in comparison with other recently published papers on V-band 90nm CMOS amplifiers [66],[69],[72],[73] is given in Table 4.1.

Table 4-1: Performance summary of this single-stage amplifier in comparison with V-band 90nm CMOS amplifiers.

\begin{tabular}{|l|l|c|c|c|c|}
\hline Ref/Year & \multicolumn{1}{|c|}{ \# of stages/Topology } & $\begin{array}{c}\text { Freq. } \\
(\mathrm{GHz})\end{array}$ & $\begin{array}{c}\mathrm{NF} \\
(\mathrm{dB})\end{array}$ & $\begin{array}{c}\text { Gain } \\
(\mathrm{dB})\end{array}$ & $\begin{array}{c}\text { IIP3 } \\
(\mathrm{dBm})\end{array}$ \\
\hline \hline$[69] / 2007$ & $2 /$ cascode (lumped) & 58 & 5.5 & 14.6 & -6 \\
\hline$[66] / 2007$ & $2 /$ common source & 62 & 6 & 12.2 & NA \\
\hline$[73] / 2008$ & $2 /$ cascode $(\mathrm{CPW})$ & 64 & 6.5 & 15 & NA \\
\hline$[72] / 2008$ & 3/ common source (lumped) & 58 & 4.4 & 15 & NA \\
\hline This Work & $1 /$ cascode (LG-CPW) & 58 & 4.1 & 10 & $>3$ \\
\hline
\end{tabular}

Assuming the proposed single-stage amplifier is cascaded to form a two-stage amplifier, the cascaded NF of the proposed amplifier would be among the lowest NF of Table 4.1. Also, the gain and IP3 of the proposed amplifier would be higher than those of Table 4.1. The power consumption of the single-stage amplifier is $15.2 \mathrm{~mW}$ from a 1.5 volt supply and is among the lowest power consumption for $60 \mathrm{GHz}$ low noise amplifiers in CMOS technology. 


\subsection{Chapter Summary}

This chapter presented and discussed a novel design approach in CMOS technology to design a V-band CMOS low-noise amplifier by using LG-CPW transmission lines as the matching elements. The use of LG-CPW elements enable achieving a wide range of impedances that facilitates the realization of optimal matching networks. The proposed single-stage amplifier demonstrated a noise figure of less than $4.1 \mathrm{~dB}$ and a gain higher than $10 \mathrm{~dB}$ in the frequency range of $57-58 \mathrm{GHz}$, as well as an input IP3 greater than $3 \mathrm{dBm}$. The noticeable frequency shift between the measured and simulated results is attributed to the accuracy of the available device models at the 60 $\mathrm{GHz}$ band frequencies. Aside from that, a demonstrated noise figure of $4.1 \mathrm{~dB}$ is lower than previously reported state of the art values of 4.4-6.5dB in $90-\mathrm{nm}$ CMOS technology. 


\section{Chapter 5}

\section{Design of a V-band CMOS Reduced-Size LG-CPW $90^{\circ}$ Coupler}

In this chapter, a V-band reduced-size quadrature coupler is designed based on the lower-ground coplanar waveguide (LG-CPW) transmission lines which were studied in Chapter 3. The coupler is implemented in a 90-nm CMOS process with 9-metal layers. Analysis and design details of the coupler as well as on-wafer measurements and electromagnetic (EM) post-layout simulation results are presented and discussed.

\subsection{Motivation}

The growing demand for high data rate wireless applications as well as the availability of $7 \mathrm{GHz}$ in the unlicensed $60 \mathrm{GHz}$ ISM band $(57-64 \mathrm{GHz})$ has created significant interest in developing low-cost $60 \mathrm{GHz}$ wireless systems. Quadrature couplers [75]-[84] are key components in radio front-ends of $\mathrm{mmW}$ systems and they are used in balanced amplifiers [85]-[87] and balanced mixers [88]. Figure 5.1 shows a $57 \mathrm{GHz}$ radio-over-fiber transceiver front-end which uses quadrature couplers in the balanced power amplifier section in order to improve the amplifier stability, the return loss, the system's reliability. In Figure 5.1, the transmit chain consists of an optical-to-electrical device, frequency up-converter which converts the base-band signal to a $57 \mathrm{GHz}$ signal, two quadrature couplers, and two mirror imaged power amplifiers (PAs). In general, the realization of quadrature couplers requires a large 
area because of their use of quarter-wavelength $(\lambda / 4)$ transmission lines. Thus, it is essential to reduce the coupler's size which significantly lowers the fabrication costs. Si-based CMOS technology is used in this study because of its low-cost and its capability for the realization of high level of integration that adaptes both the digital and RF circuits on a single chip. Although CMOS technology has many advantages, its design rules present a major challenge for the realization of high impedance lines which are needed for size reduction. This challenge motivates the design effort to develop a reduced-size V-band CMOS branch-line coupler. 


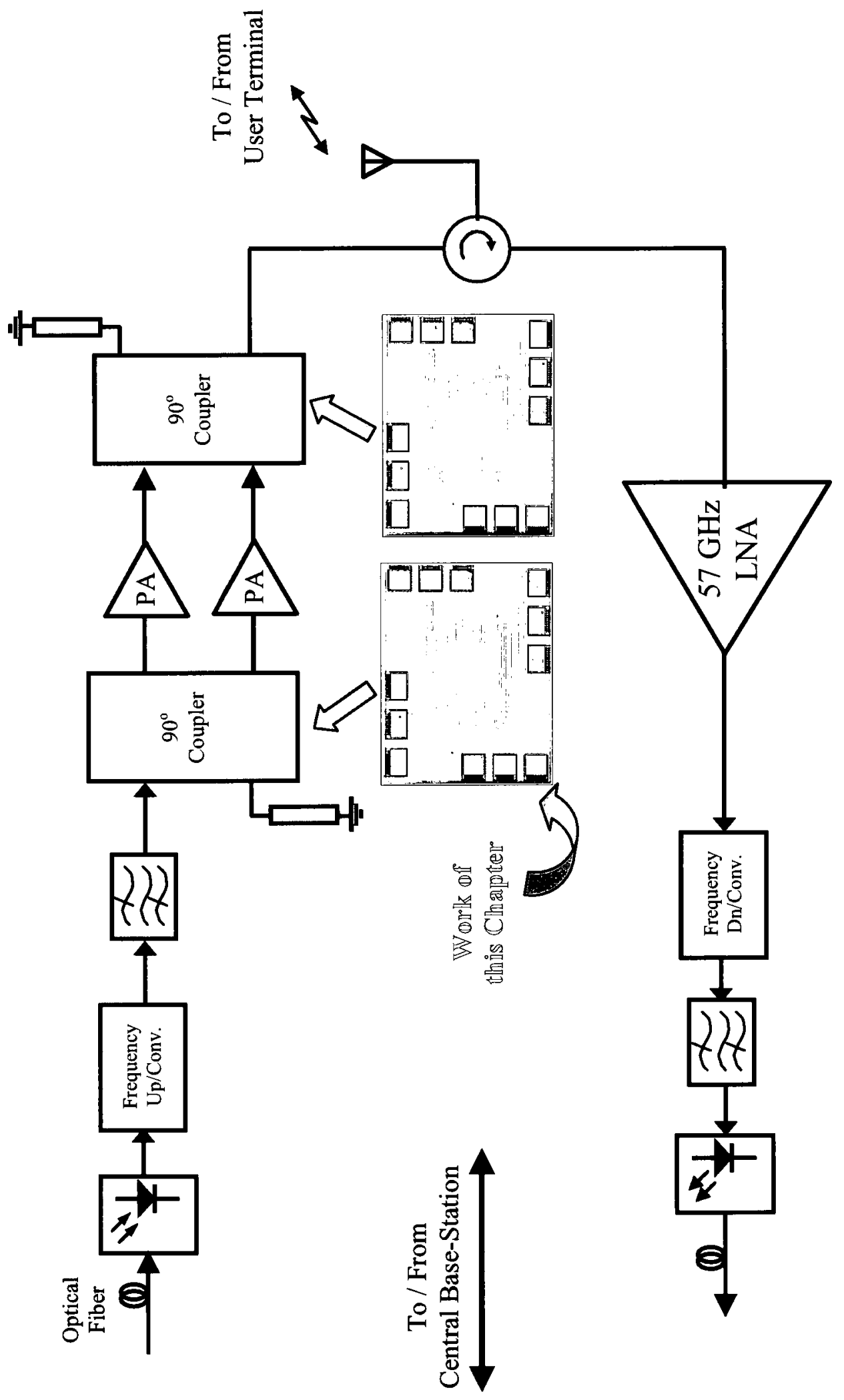

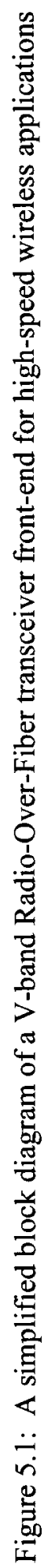




\subsection{Branch-Line Couplers in CMOS Technology}

A branch-line coupler is a four-port microwave component which provides a 90 degree phase difference between port-2 (direct port) and port-3 (coupled port) where port-4 (isolated port) is connected to a $50-\mathrm{ohms}$ load. The coupler consists of four quarter wavelength $\lambda / 4$ transmission line elements as shown in Figure 5.2. Conventional couplers tend to consume an expensive chip area because of the use of $\lambda / 4$ elements. Many efforts have been made to reduce the size of these couplers by using high impedance lines that are capacitively loaded at their ends [89]-[94]. However, having high impedances in CMOS technology could be a challenge due to the technology design rules, also, obtaining lumped capacitors with the required values are not always available in all CMOS design kits. Furthermore, the devices of most of the design kits are validated up to $20 \mathrm{GHz}$ only.

The effort in this work is to design a compact branch-line coupler that achieves a significant size reduction while maintaining the CMOS process design rules. The proposed branch-line coupler utilizes LG-CPW high impedance transmission line elements, which are loaded at their ends with metal-insulator-metal (MIM) capacitors. In this coupler, the ground-plane strips of the LG-CPW lines are also used as the lower plates of the MIM capacitors. 


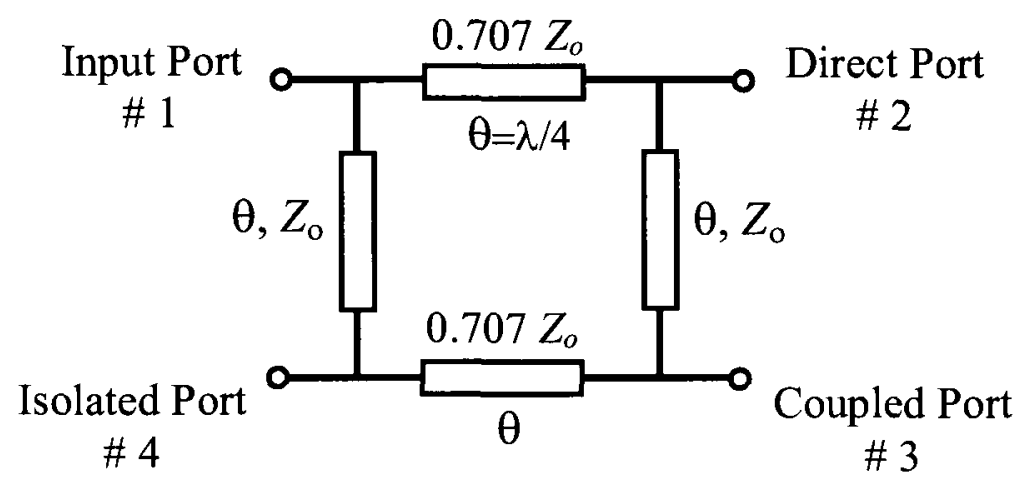

Figure 5.2: A conventional branch-line coupler

\subsubsection{CMOS Capacitively Loaded LG-CPW Transmission Lines}

The elements of the branch-line coupler of this work are implemented by using loaded LG-CPW transmission lines. In a CMOS LG-CPW transmission line structure, the signal line is implemented on the top metal layer, whereas the ground-plane strips are implemented on a lower metal-layer. These lines offer several advantages over conventional CPW lines. First, LG-CPW structures enable achieving highimpedances without reducing the widths of their signal-lines, thus, minimizing the dependency of the structure on the manufacturing tolerance; and reducing the conductor loss which lowers the signal attenuation. Second, the area above the ground-plane strips can be used with another metal layer to form the MIM capacitors for loading the lines without increasing the chip-area. Increasing the line's capacitance and the inductance (due to the high impedance) reduces the signal's phase velocity $v_{\mathrm{p}}$ which leads to a reduced wavelength $\lambda$, which is given by: 


$$
\begin{gathered}
\lambda=\frac{v_{p}}{f}, \\
v_{p}=\frac{1}{\sqrt{L C}},
\end{gathered}
$$

where $L$ and $C$ are the inductance and capacitance per unit length, respectively. Reducing the wavelength of the quarter-wavelength elements contributes to the size reduction of the coupler. Figure 5.3 shows a capacitively loaded CMOS LG-CPW transmission line.

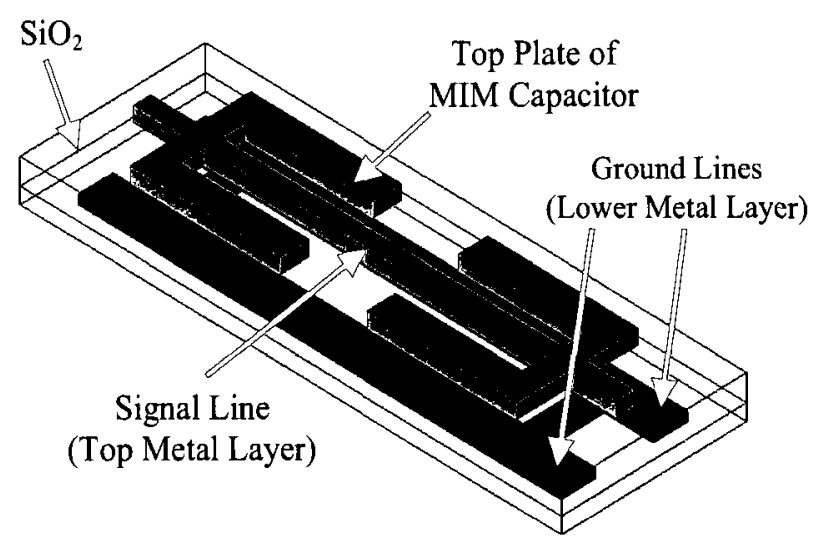

Figure 5.3: Capacitively loaded CMOS LG-CPW transmission line element

\subsection{Analysis of Reduced-Size Transmission Lines}

Figures 5.4(a) and 5.4(b) show a conventional transmission line section and its equivalent capacitively loaded line. In Figure 5.4 (b), $\theta$ and $Z$ are the electrical length 
and the characteristic impedance of the shortened line, and $C$ is capacitance of the capacitive loads at the ends of the shortened line.

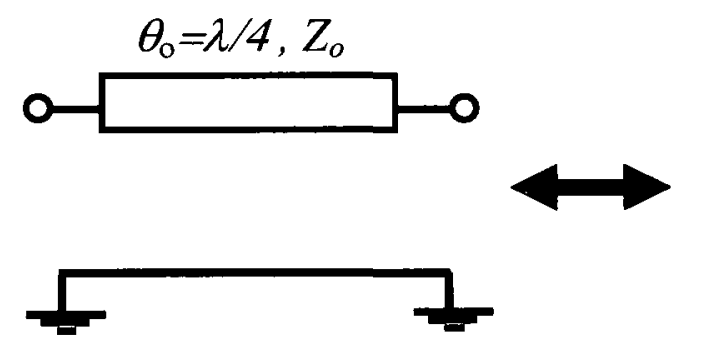

(a)

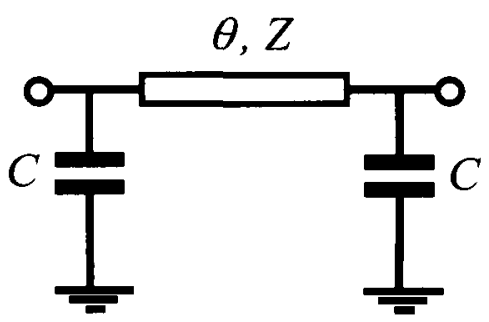

(b)

Figure 5.4: Schematic representation of (a) conventional transmission line section, (b) capacitively loaded line

The characteristic impedance, $Z$, and the loading capacitance, $C$, can be determined by equating the $A B C D$-matrices of the two-port networks of Figures 5.4(a) and 5.4(b) which are given by:

$$
\begin{aligned}
{[A B C D]_{a} } & =\left[\begin{array}{cr}
\cos \theta_{o} & j Z_{o} \sin \theta_{o} \\
j \frac{1}{Z_{o}} \sin \theta_{o} & \cos \theta_{o}
\end{array}\right] \\
{[A B C D]_{b} } & =\left[\begin{array}{cc}
1 & 0 \\
j B & 1
\end{array}\right] \cdot\left[\begin{array}{cc}
\cos \theta_{o} & j Z_{o} \sin \theta_{o} \\
j \frac{1}{Z_{o}} \sin \theta_{o} & \cos \theta_{o}
\end{array}\right] \cdot\left[\begin{array}{cc}
1 & 0 \\
j B & 1
\end{array}\right] \\
& =\left[\begin{array}{ll}
\cos \theta-B \cdot Z \sin \theta & j Z \sin \theta \\
j \frac{\sin \theta}{Z}\left(1-Z^{2} B^{2}+2 Z \cdot B \cot \theta\right) & \cos \theta-B \cdot Z \sin \theta
\end{array}\right]
\end{aligned}
$$

where $B=\omega C$. From (5.2) and (5.3) the parameters $Z$ and $C$ can be expressed as 


$$
Z=Z_{o} \frac{\sin \theta_{o}}{\sin \theta}
$$

and

$$
C=\frac{1}{\omega}\left(\frac{\cos \theta}{Z_{o} \sin \theta_{o}}-\frac{\cos \theta_{o}}{Z_{o} \sin \theta_{o}}\right)
$$

From (5.4), by choosing $Z$ to be twice the value of $Z_{0}$ and with $\theta_{o}=90$ degrees, a line reduction of $66 \%$ can be achieved (i.e., $\theta=30$ degrees).

\subsection{Design of a Reduced-Size $90^{\circ}$ Coupler using CMOS LG-CPW Elements}

In this design, the impedances and electrical lengths of the longitudinal and transverse branches of the quadrature coupler can be estimated from (5.4), thus,

$$
\frac{\sin \theta_{T}}{\sin \theta_{L}}=\sqrt{2} \frac{Z_{L}}{Z_{T}}
$$

where $\theta_{L}, \theta_{T}, Z_{L}$, and $Z_{T}$ are the electrical lengths and the impedances of the longitudinal and the transverse branches of the coupler, respectively. Making $Z_{\mathrm{T}}$ the same as $Z_{\mathrm{L}}$ to facilitate the fabrication of the coupler, (5.6) can be written as

$$
\sin \theta_{T}=\sqrt{2} \sin \theta_{L}
$$

From (5.7), choosing $\theta_{\mathrm{L}}=\lambda / 12$ results in $\theta_{\mathrm{T}}=\lambda / 8$. With $Z_{\mathrm{T}}$ the same as $Z_{\mathrm{L}}$ and $Z_{0}=100$ ohms, the impedance of each branch in the coupler becomes 70.7 ohms. To accomplish the required length reduction, the capacitance at each corner of the 
coupler needs to be $0.1025 \mathrm{pF}(0.047 \mathrm{pF} / / 0.055 \mathrm{pF})$ at $60 \mathrm{GHz}$. Figure 5.5 shows the circuit schematic of the capacitively loaded coupler using ideal transmission line elements and lumped capacitors. The simulated scattering parameters of the coupler shown in Figure 5.5 are presented in Figure 5.6. The results of Figure 5.6 reveal that the coupling between the input and the direct ports (S21) is $-3 \mathrm{~dB}$ at $60 \mathrm{GHz}$ as expected since the transmission lines are lossless. The coupling between the input and the coupled ports is also $-3 \mathrm{~dB}$, and the isolation between the input and the isolated port is $-60 \mathrm{~dB}$. The simulated phase difference between the direct and the coupled ports of the coupler is shown in Figure 5.7. From Figure 5.7, the phase difference between the output ports of the coupler is 90 degrees. 


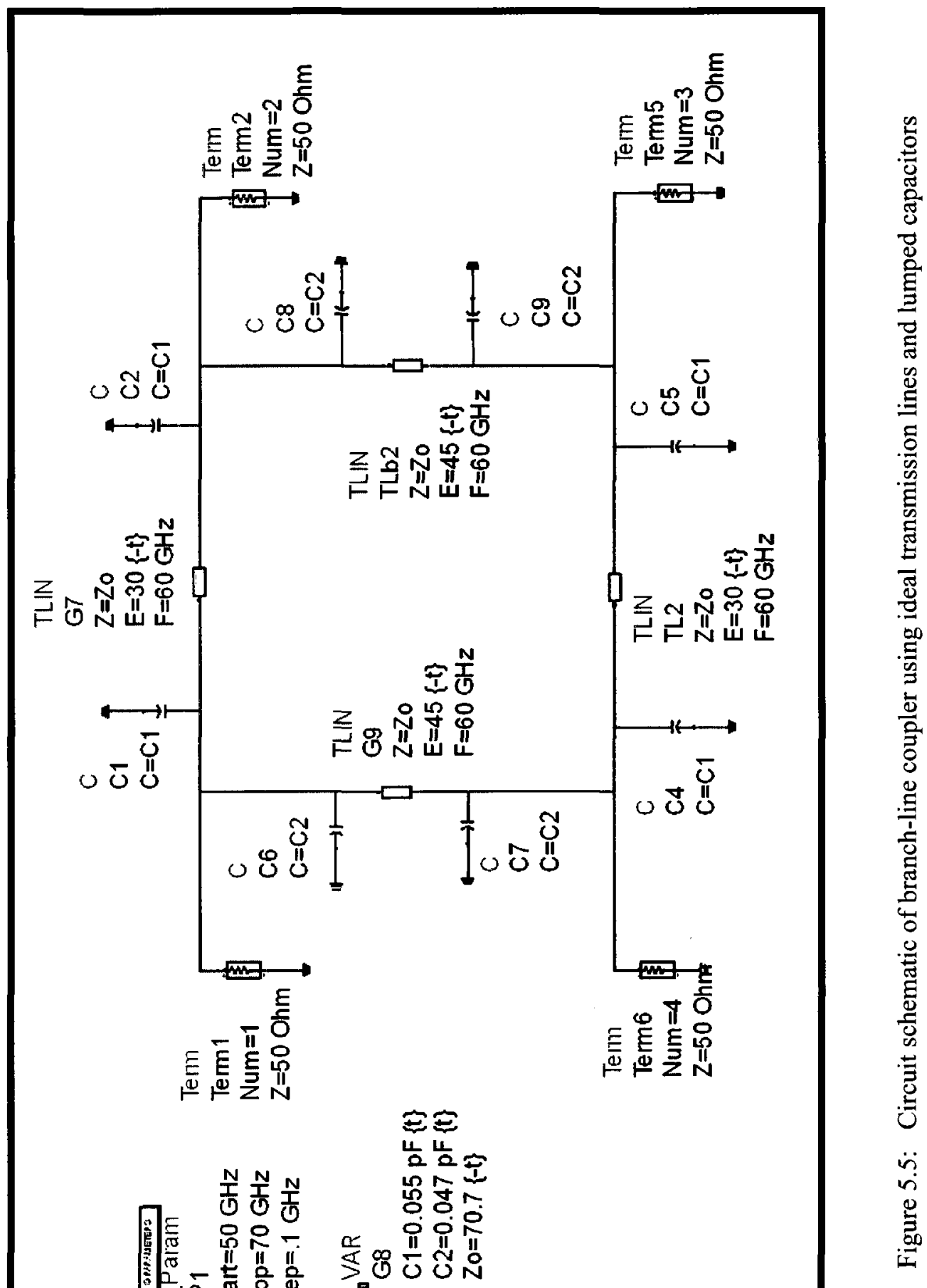




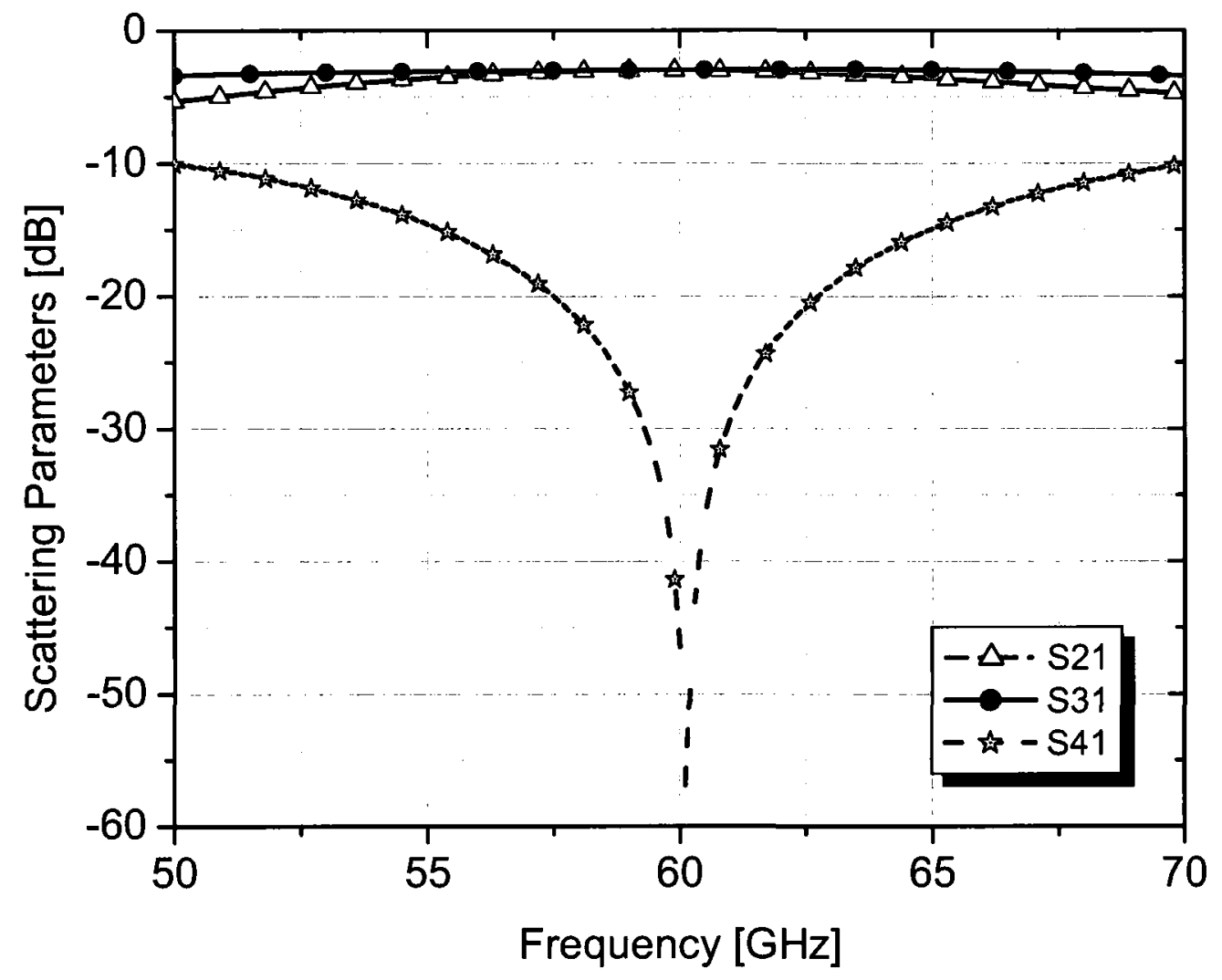

Figure 5.6: Simulated S-parameters of the branch-line coupler (ideal transmission lines and lumped capacitors) 


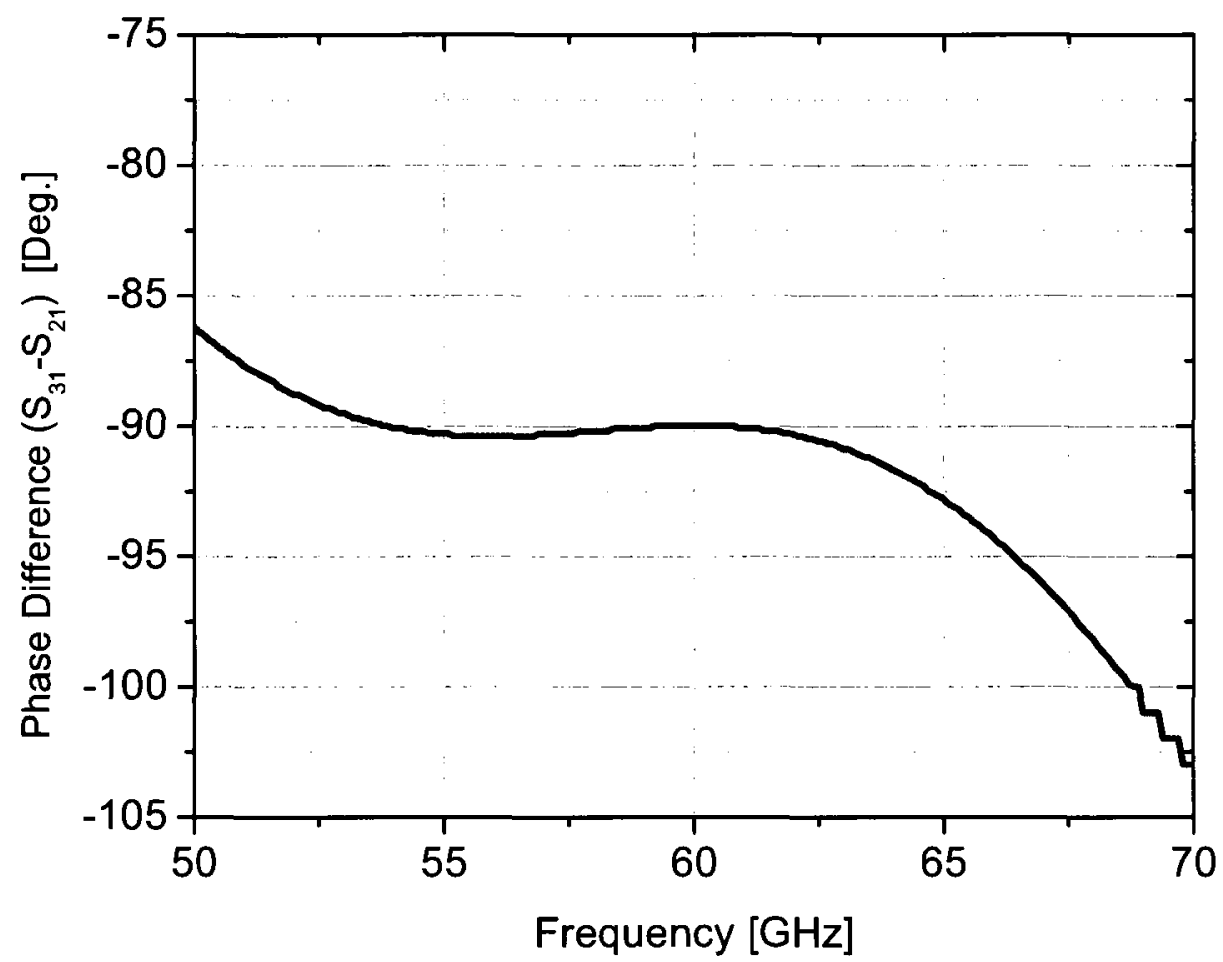

Figure 5.7: Simulated phase difference of the branch-line coupler (ideal transmission lines and lumped capacitors) 


\subsection{Layout of the CMOS LG-CPW $90^{\circ}$ Branch-Line Coupler}

The layout of the coupler shown in Figure 5.5 is implemented by replacing the ideal transmission line elements and lumped capacitors with LG-CPW elements and MIM capacitors. Figure 5.8 shows the 3-D view of the layout of the proposed coupler. In Figure 5.8, the signal lines are implemented on the top metal-layer M9 while the ground-plane strips of the LG-CPW elements are implemented on M7. The width of the signal-lines is $8 \mu \mathrm{m}$, and the width of the ground-plane strips is $12 \mu \mathrm{m}$. The MIM capacitors are formed by the two metal layers M9 and M7 and are located at the corners of the coupler. The size of the fabricated coupler without test the pads is 355$\mu \mathrm{m} \times 287-\mu \mathrm{m}$ which is approximately $73 \%$ size-reduction in comparison with layout of a conventional CPW coupler. The coupler's size is relatively smaller than those previously published [91]-[96]. Figure 5.9 shows the microphotograph of the coupler chip which was fabricated [97]-[98] in a 90nm 9-metal layers CMOS technology. 


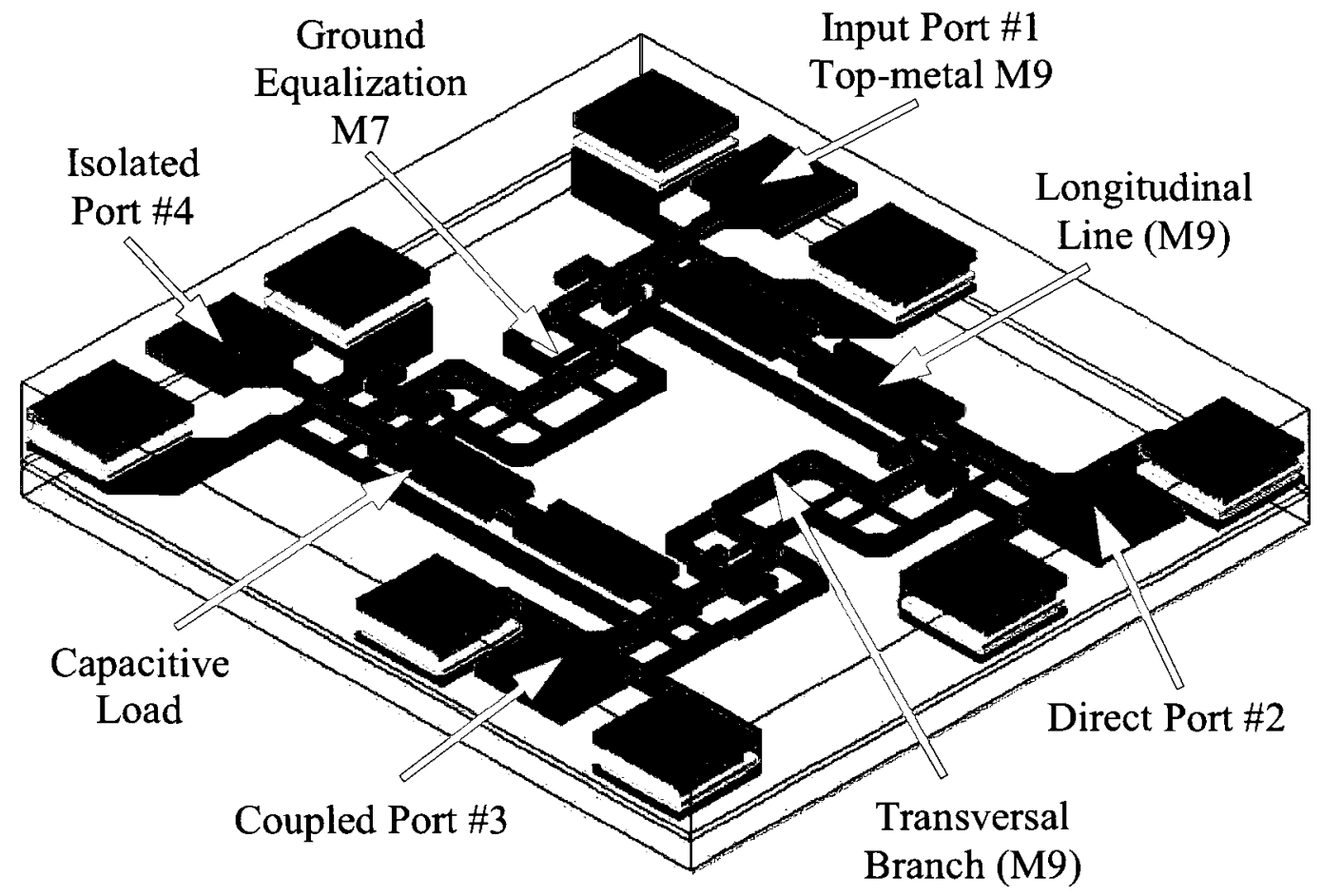

Figure 5.8: 3-D view (Si-substrate is not shown) of the layout of the $60 \mathrm{GHz}$ branchline coupler

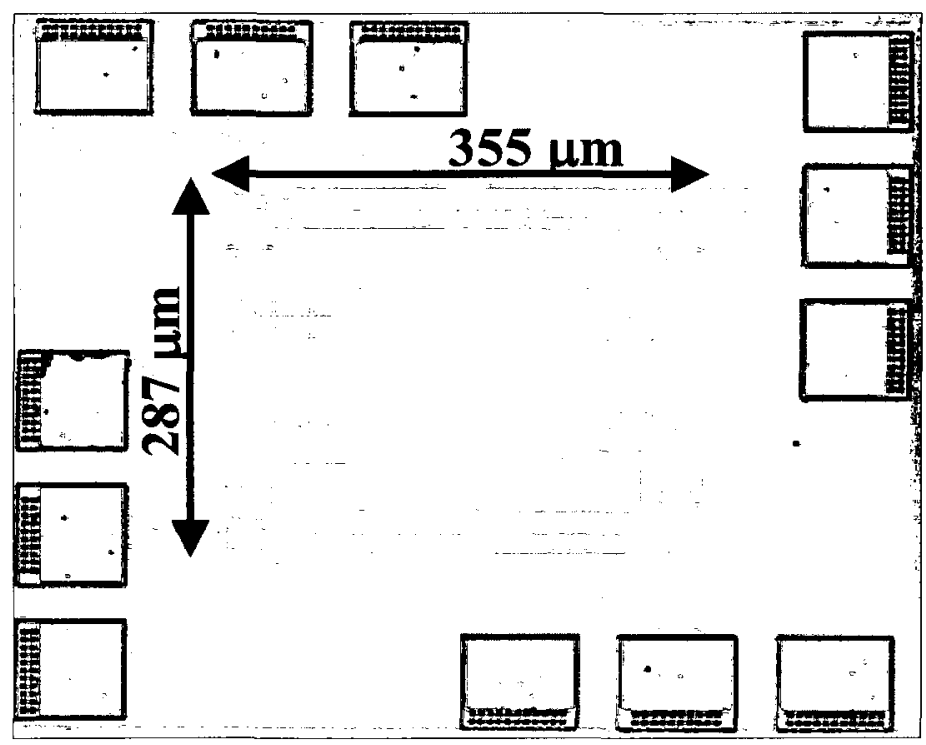

Figure 5.9: Microphotograph of the fabricated $60 \mathrm{GHz}$ CMOS reduced-size coupler 


\subsection{Design Flow of a CMOS LG-CPW Branch Line Coupler}

The design methodology of the CMOS LG-CPW branch line coupler of this study is summarized in the design flow shown in Figure 5.10.

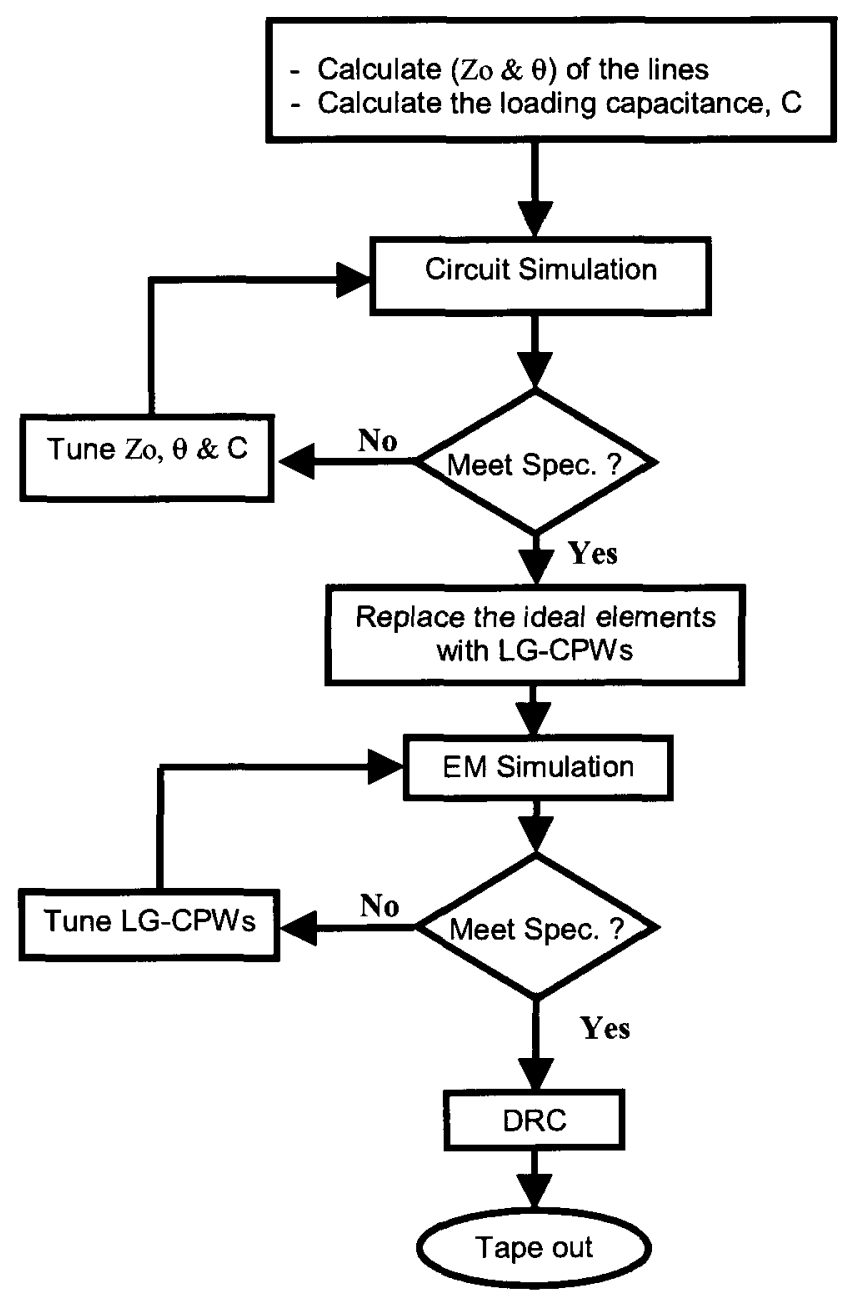

Figure 5.10: Design flow of the LG-CPW Low Noise Amplifier 


\subsection{Experimental and Post-layout Simulation Results}

The on-wafer measurements of the coupler were carried out using $67 \mathrm{GHz}$ vector network analyzer (Agilent E8361A), and the EM simulation was performed by using an electromagnetic simulator (Agilent ADS momentum). Figure 5.11 shows the measured and post-layout simulated S-parameters of the $60 \mathrm{GHz}$ CMOS LG-CPW branch line coupler. From Figure 5.11, the measured insertion loss between the input and the direct ports is about $1.8 \mathrm{~dB}$ at $60 \mathrm{GHz}$, and that between the input and the coupled ports is about $2.5 \mathrm{~dB}$. The measurements also show an isolation of $16.5 \mathrm{~dB}$ at $60 \mathrm{GHz}$ between the input and the isolated ports. The measurement and the postlayout simulation results closely agree over the $60 \mathrm{GHz}$ band.

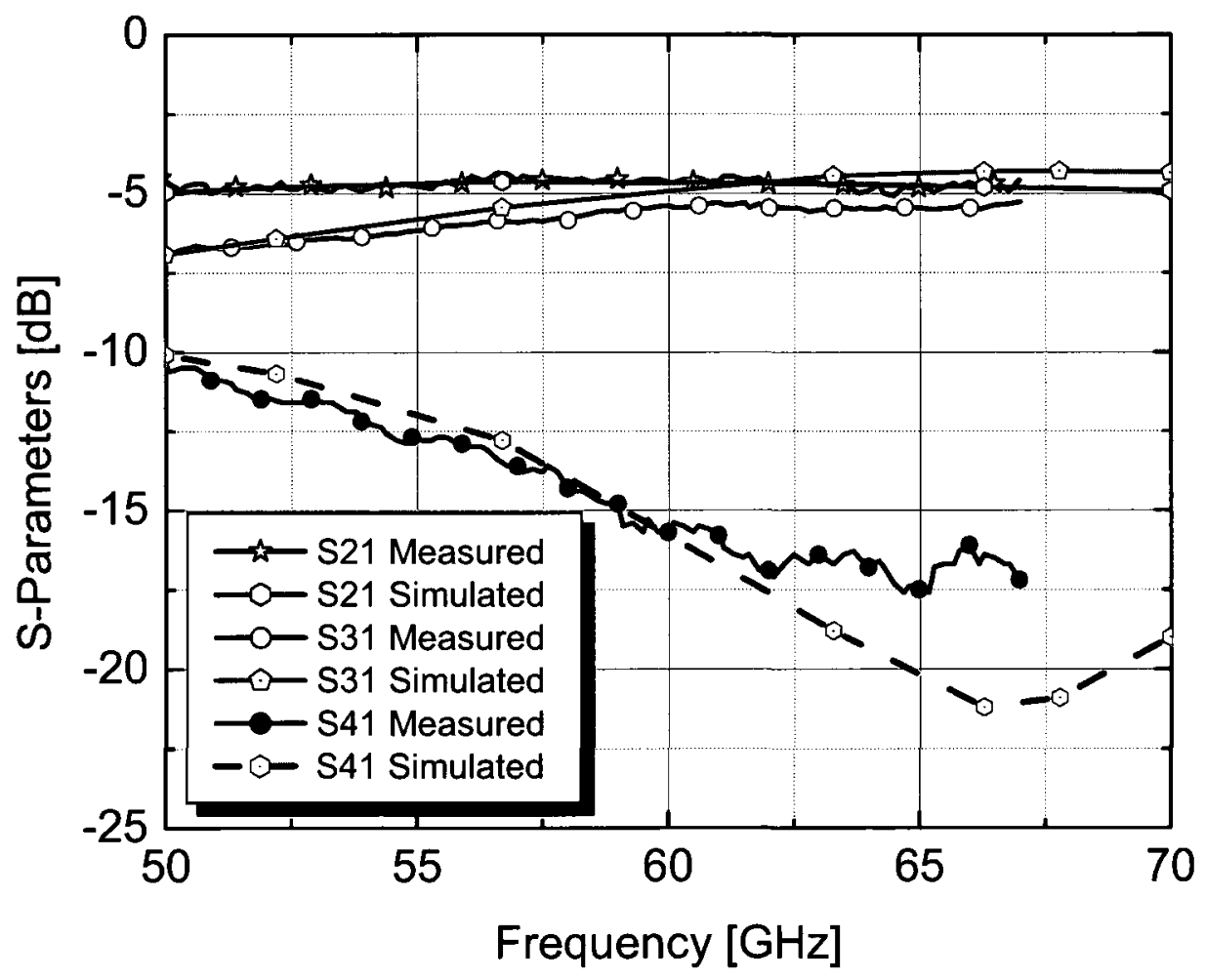

Figure 5.11: Measured and post-layout simulated S-parameters of the LG-CPW branch line coupler 
Figure 5.12 shows the measured and the post-layout simulated phase-difference between the direct and coupled ports (phase $\left(\mathrm{S}_{31}-\mathrm{S}_{21}\right)$ ). From Figure 5.12, the measured phase $\left(\mathrm{S}_{31}-\mathrm{S}_{21}\right)$ is about 2 degrees off from the required 90 degrees at 60 GHz. The measurements are better than the post-layout simulation results. The discrepancy between the measured and simulated results is due to the accuracy of the substrate parameters of the technology's design kit. Figure 5.13 shows the measured and simulated amplitude imbalance between the direct and coupled ports. The results of Figure 5.13 reveal that the amplitude imbalance is about $0.7 \mathrm{~dB}$ at $60 \mathrm{GHz}$, the measured amplitude imbalance is off by $0.5 \mathrm{~dB}$ from the simulated value at $60 \mathrm{GHz}$. The experimental characterization of the coupler showed a down shift in the frequency response of the return loss at all the ports of the coupler. Such a shift in frequency is due to the accuracy of the substrate parameters of the technology's design kit. However, the return loss at any port is less than $-15 \mathrm{~dB}$ in the entire 60 $\mathrm{GHz}$ band $(57-64 \mathrm{GHz})$. 


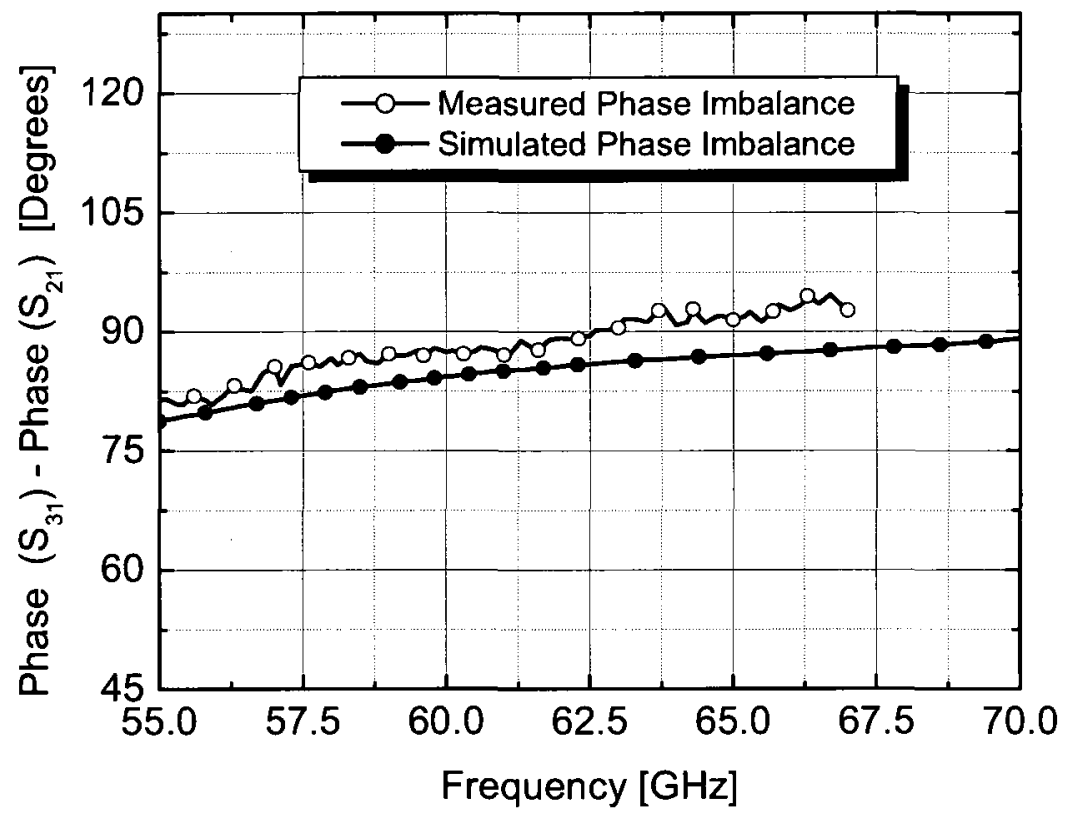

Figure 5.12: Measured and post-layout simulated phase-difference of the LG-CPW branch line coupler 


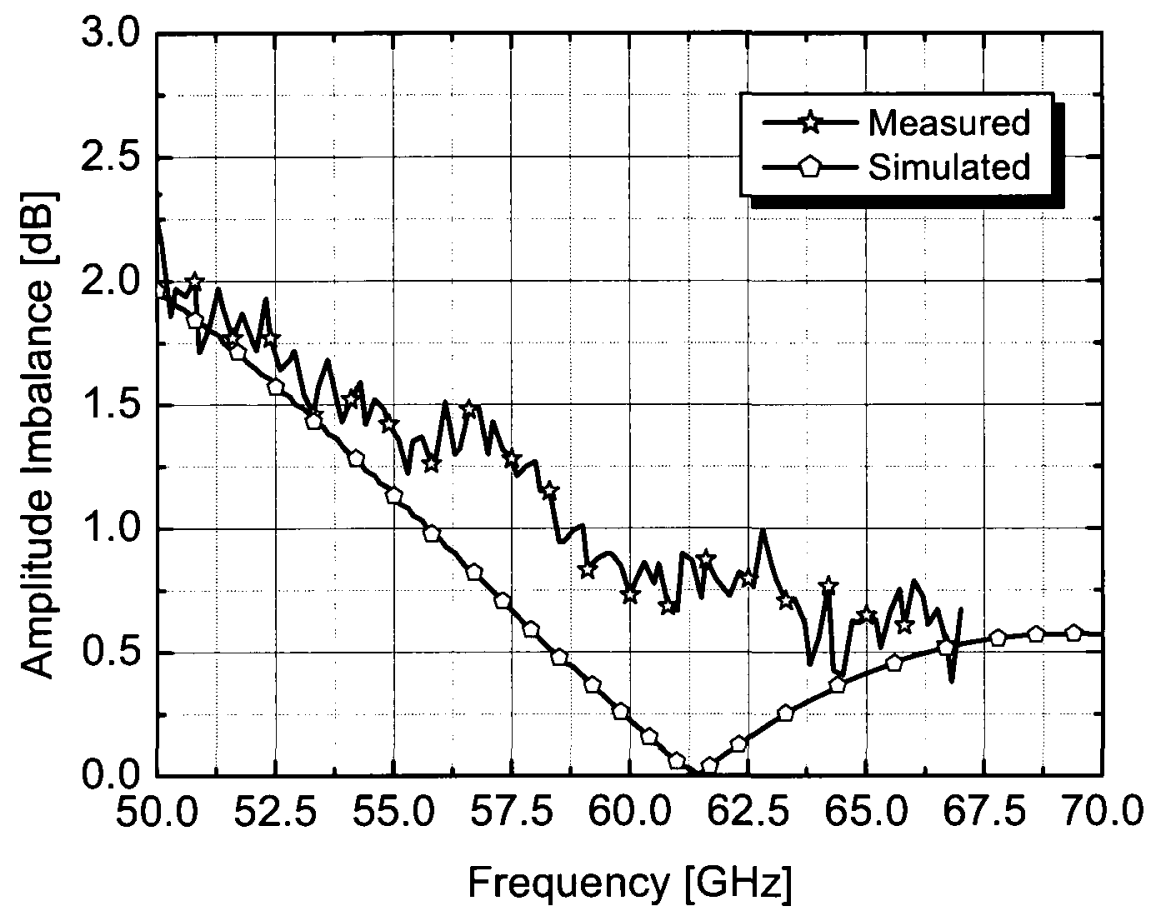

Figure 5.13: Measured and post-layout simulated amplitude-imbalance of the LGCPW branch line coupler 


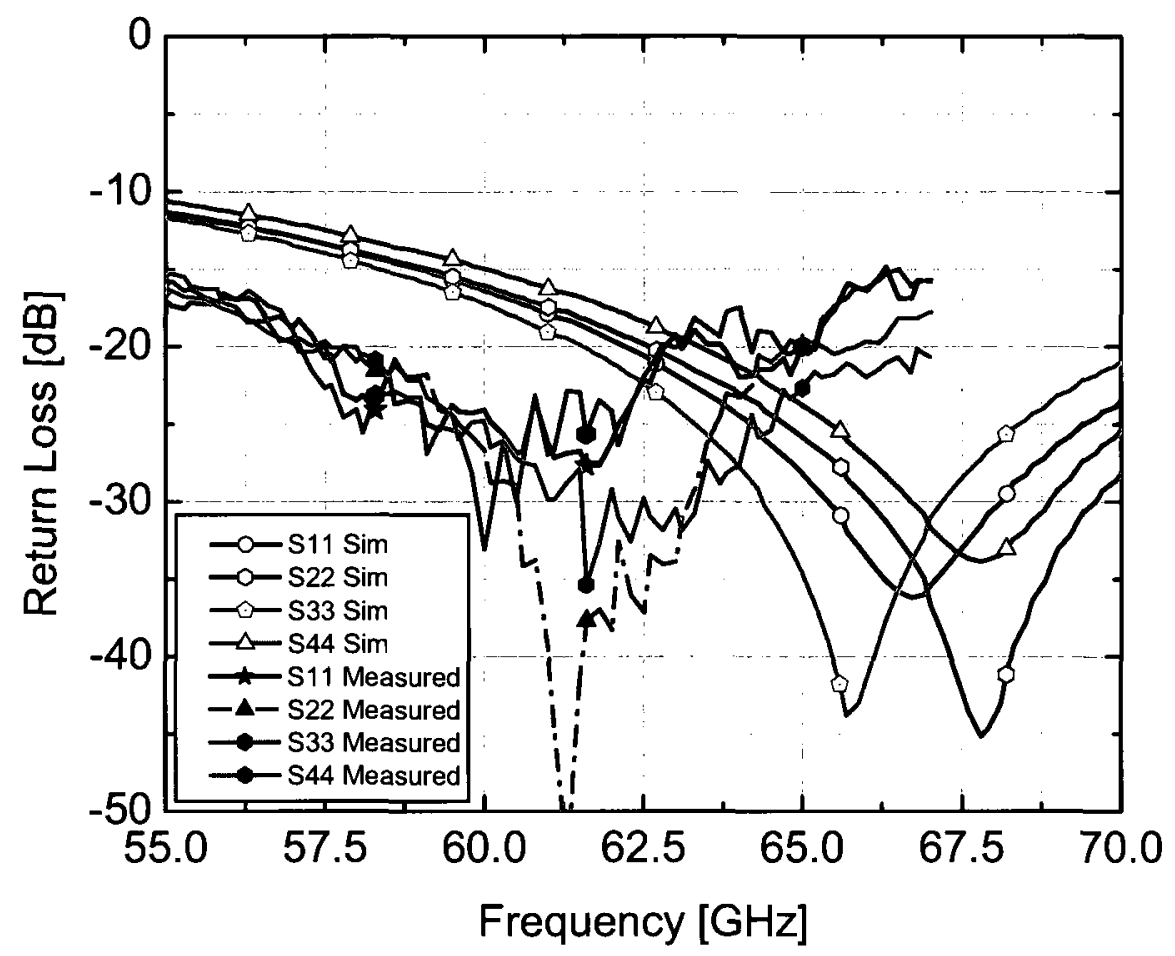

Figure 5.14: Measured and post-layout simulated return loss of the EC-CPW branch line coupler 


\subsection{Chapter Summary}

A $60 \mathrm{GHz}$ band reduced-size LG-CPW branch line coupler has been designed and developed in a 90nm 9-metal layers CMOS technology. An area-reduction of more than $70 \%$ in comparison with a conventional CPW coupler was achieved. The sizereduction was implemented by using capacitively loaded LG-CPW transmission lines, which enable the realization of high-impedance lines, and MIM capacitors. The measurements and the EM post-layout simulation results of the amplitude imbalance and the phase difference closely agree at $60 \mathrm{GHz}$ and reasonably agree over the 60 $\mathrm{GHz}$ band. The measured results of the return losses indicated a shift in the frequency response, which is attributed to the accuracy of the substrate parameters of the CMOS design kit. However, the return losses are below $-15 \mathrm{~dB}$ over the entire $60 \mathrm{GHz}$ band for all the ports.

The chip size of the fabricated coupler is $355 \mu \mathrm{m} \times 287 \mu \mathrm{m}$, which is smaller than that of all published data on $60 \mathrm{GHz}$ couplers. The coupler of this chapter is well suited for use in the RF front-ends of $\mathrm{mmW}$ radio systems for high-speed short-range communication links. 


\section{Chapter 6}

\section{Conclusions and Future Work}

This thesis has investigated the development of V-band Si-based CMOS lowerground coplanar waveguide (LG-CPW) transmission lines and their successful implementation in the development of a V-band CMOS low noise amplifier and a 60 $\mathrm{GHz}$ CMOS reduced-size branch-line coupler.

The proposed transmission lines provide design flexibility to achieve a wide range of impedances which facilitates the design of the matching networks and the passive components in $\mathrm{mmW}$ radio front-ends, to achieve optimal system performance. The proposed transmission lines also mitigate the metal design rule restrictions of the CMOS technology, which could hinder the performance of CMOS systems.

The low noise amplifier of this research is based on the use of LG-CPW transmission lines as matching elements to enable the realization of the needed impedances for achieving optimal noise performance. To the author's best knowledge, the proposed amplifier is one of the lowest noise figure and the highest linearity that have been achieved with a 90-nm CMOS technology at $\mathrm{mmW}$ frequencies in V-band; the power consumption is also among the lowest power consumption of low noise amplifiers in $90-\mathrm{nm}$ CMOS. The experimental characterization of the proposed amplifier revealed that the noise figure is less than 
$4.2 \mathrm{~dB}$ at $57 \mathrm{GHz}$, the gain is greater than $10 \mathrm{~dB}$, the input third-order intercept point is close to $3 \mathrm{dBm}$, and the power consumption is about $15.2 \mathrm{~mW}$ from a $1.5 \mathrm{~V}$ supply.

The proposed quadrature branch-line coupler utilized capacitively loaded LGCPW elements to reduce the physical size of the coupler's quarter-wavelength branches. The coupler's size is $0.102 \mathrm{~mm}^{2}$ which is more than $75 \%$ area reduction compared to conventional CPW branch-line couplers. The size of the proposed coupler is the smallest known $60 \mathrm{GHz}$ couplers in CMOS technology. The coupler achieved a measured phase difference of less than 3 degrees at $60 \mathrm{GHz}$ and an amplitude imbalance of less than $2.5 \mathrm{~dB}$ over the frequency range of $57-64 \mathrm{GHz}$.

The research of this thesis demonstrated the potential of using LG-CPW transmission lines to enhance the performance and reduce the size of the active and the passive RF building blocks in CMOS technology. This research completes the thesis objective and presents a considerable contribution to the field of mmW CMOS passive and active components as well as systems-on-chip.

The proposed LG-CPW transmission lines will be very useful in the design of reduced-size mmW CMOS balanced amplifiers, balanced mixers, phase shifters, power combiners/splitters, filters, and antenna feeding networks.

Future research can be carried out to develop accurate device models for CMOS technology at frequencies in the V-band. Also, utilizing the branch-line coupler of this work, the design of a $60 \mathrm{GHz}$ band balanced power amplifier in $90 \mathrm{~nm}$ CMOS technology can be undertaken. 


\section{References}

[1] Meinel, H.H., "Commercial applications of millimeter waves History, Present Status, and Future Trends", IEEE Transaction on Microwave Theory and Technique, vol. 43, no. 7, July 1995, pp. 1639-1653.

[2] Luis M. Correia and Ramjee Prasad, "An overview of wireless broadband communication", IEEE Communications Magazine, Jan. 1997, pp. 28-33.

[3] Yoneyama, T., "Millimeter-Wave Research Activities in Japan", IEEE Trans. MTT, June 1998, Vol. 46, No. 6.

[4] Taimoto, Y., Thara, T., "Research Activities on Millimeter-Wave Indoor Communicaton systems in Japan", 1993 IEEE MTT-S Digest, pp. 673.

[5] Smulders, P., "Exploiting the $60 \mathrm{GHz}$ Band for Local Wireless Multimedia Access: Prospects and Future directions", Commun. Mag. IEEE, Jan. 2002, Vol. 40, Issue 1, pp. 140-147.

[6] Choi, S., et al., "A $60 \mathrm{GHz}$ Point-to-Multioint Millimeter-Wave Fiber-Radio Communication System", IEEE Trans. MTT, May 2006, Vol. 54, No. 5.

[7] Long, John R., "Can Silicon Catch the Millimeter Wave?," $35^{\text {th }}$ European Microwave Conference 2005..

[8] O'Hara, B., Petrick, A., 802.11 Handbook: A Designer's Companion, IEEE Press, 1999.

[9] Chong, C., Kumar, S., "Sensor Networks: Evolution, Opportunities, and Challenges", Proc. IEEE, Vol. 91, No. 8, August 2003.

[10] Akylindiz, I., et al., "A Survey on Sensor Networks", IEEE Comm. Mag., August, 2002.

[11] Wen, C., "Coplanar waveguide: A surface strip transmission line suitable for nonreiprocal gyromagnetic device applications," IEEE Trans. Microwave Theory Tech, vol. 17, pp. 1087-1090, Dec. 1969.

[12] Ghione, G., and Nadli, C., "Coplanar Waveguides for MMIC applications: Effect of upper shielding, conductor backing, finite-extent ground planes, and line-to-line coupling," IEEE Tran. Microwave Theory Tech., fol. MTT-35, pp60-267, Mar. 1987. 
[13] Gillick, M., et al., "Uniplanar techniques for monolithic microwave integrated circuits", Electronics and Communication Engineering Journal, August 1994, pp. 187-194.

[14] Simons, R., Coplanar Waveguide Circuits, Components, and Systems, Wiley and Sons, New York, $1^{\text {st }}$ Ed., 2001.

[15] Wolff, Ingo, Coplanar Microwave Integrated Circuits, Wiley Interscience, A John Wiley \& Sons, Inc., Hoboken, New Jersey, 2006.

[16] Doan, H. Chinh, et al., "Design of CMOS for $60 \mathrm{GHz}$ Applications", IEEE International Solid-State Circuit Conference 2004.

[17] Doan, C. H., Emami, S., Sobel, D. A., Niknejad, A. M., and Brodersen, R. W., "Design Considerations for $60 \mathrm{GHz}$ CMOS Radios", IEEE Communication Magazine, Dec 2004, vol. 42, no. 12, pp. 132-140.

[18] Doan, H. Chinh, Niknejad, A., et al., "Design Considerations for $60 \mathrm{GHz}$ CMOS Radios", IEEE Communications Magazine, December 2004

[19] Doan, H. Chinh, et al., "Millimeter-Wave CMOS Design", IEEE Journal of Solid-State Circuits, January 2005, vol. 40, no. 1.

[20] Behzad Razavi, RF Microelectronics, Prentice-Hall, Inc., 1998.

[21] Haroun, I., Hsu, Y-C., Wight, J., Plett, C., "A CMOS Low-Noise Amplifier with VPW Matching Elements for 60-GHz-band Gbit/s Wireless Systems," 2009 Asia Pacific Microwave Conference, Singapore, December 2009.

[22] Haroun, I., Wight, J., Plett, C., Fathy, A., "A V-band 90-nm CMOS Low-Noise Amplifier with Modified CPW Transmission Lines for UWB Systems," IEEE Radio and Wireless Symposium (RWS-2010), New Orleans, January 2010.

[23] Haroun, I., Wight, J., Plett, C., Fathy, A., "Multi-band 700MHz/2.4GHz/60GHz RF Front-End for Radio-over-Fiber Base-Stations," IEEE Radio and Wireless Symposium (RWS-2010), New Orleans, January 2010.

[24] Haroun, I., Wight, J., Plett, C., Fathy, A., "A V-band 90-nm CMOS Low-Noise Amplifier with Modified CPW Transmission Lines for UWB Systems," Invited Paper, 2010 IEEE Symposium on SiRF, New Orleans, January 2010.

[25] Yue, P.,, et al, "Study on Substrate Effects of Silicon-Based RF Passive Components", 1999 IEEE MTT-S Digest, pp. 1625.

[26] Reyes, A., et al, "Silicon as a Microwave Substrate", 1994 III MTTS-Digest, pp. 1759. 
[27] Hasegawa, H., et al., "Properties of microstrip line on Si-SiO2 system," IEEE Tran. Microwave Theory Tech., vol. MTT-19, pp. 869-881, Nov. 1971

[28] Zagge, S., "Characterization of the broad-band transmission behavior of interconnections on silicon substrate," IEEE Trans. Comp., Hybrids, Manufact. Technol., vol. 16, Nov. 1993.

[29] Gupta, K., Garg, R., Bahl, I., Bhartia, P., Microstrip Lines and Slotlines, Artch House, Norwood, MA, 1996.

[30] Pozar, David M., Microwave Engineering, $3^{\text {rd }}$ Edition, pp. 143, John Wiley \& Sons, Inc, 2005.

[31] Hettak, K., Morin, G., Stubbs M., "The Integration of Thin-Film Microstrip and Coplanar Technologies for Reduced-Size MMICs", IEEE Trans. MTT, January 2005 , vol. 53 , no. 1 .

[32] Hasegawa T., et al, "Characteristics of Valley Microstrip Lines for use in Multilayer MMIC's", IEEE Microwave and Guided Wave Letters, October 1991, vol. 1, no. 10.

[33] Collin, E., Foundations for Microwave Enginnering, Second Edition, Wiley Interscience, A John Wiley \& Sons, Inc., Hoboken, New Jersey, 2001.

[34] Lin, Y-S, et al., "Ultralow-Loss and Broadband Microwmachined Transmission Line Inductors for 30-60 GHz CMOS RFIC Application," IEEE Trans. on Electron Devices, vol. 54, no. 9, 2007, pp. 2513-2519.

[35] Chang, J-F., "An Analysis of Substrate Effects on Transmission Lines for Millimeter-wave CMOS RFIC Applications," Microwave and Optical Letters, vol. 50, no. 2,2008 , pp. $3419-324$.

[36] Milanovic, V., et al., "Microwmachined Microwave Transmission Lines in CMOS Technology," IEEE Trans. on MTT, vol. 45, no. 5, 1979, pp. 630-635.

[37] Cheung, T., et al. "Shielded Passive Devices for Silicon-Based Monolithic Microwave and Millimeter-Wave Integrated Circuits," IEEE JSSC, vol. 41, no. 5, 2006, pp. 1183-1200.

[38] Park, J-H., et al., "Novel Micromachined Coplanar Waveguide Transmission Lines for Application in Millimeter-Wave Circuits," Jpn. J. Appl. Phys. Vol. 39, (2000), part 1, no. 12B, pp. 7120-7124.

[39] Cheung, T., "On-Chip Interconnect for mm-Wave Applications Using an AllCopper Technology and Wavelength Reduction," ISSCC 20003, Section 22. 
[40] Kaddour, D., "Design Guidelines for Low-Loss Slow-Wave Coplanar Transmission Lines in RF-CMOS Technology," Microwave and Optical Tech. Letters, vol. 50, no. 12, 2008, pp. 3029-3036.

[41] Warns, C., "Transmission Lines and Passive Elements for Multilayer Coplanar Circuits on Silicon," IEEE Trans. on MTT, vol. 46, no. 5, 1998, pp. 616-622.

[42] Lai, J., et al., "Analysis of On-Chip Asymmetric Coaxial Waveguide Structure for Chip Area Reduction," Japanese Journal of Applied Physics, vol. 46, no. 4B, 2007, pp. 2261-2264.

[43] Lai, I., "High-Q Slow-Wave Transmission Line for Chip Area Reduction on Advanced CMOS Processes," IEEE Int. Conf. on Microelectronic Test Structures, 2009, pp. 192-195.

[44] Zhang, Lu, et al, "Effects of the Thin-Film Metal Ground Embedded in OnChip Microstrip Lines", IEEE Microwave and Wireless Components Letters, June 2007, vol. 17, no. 6 .

[45] Schnieder, F., et al., "Coupling Between Neighboring CPW's in MMIC's", IEEE Microwave and Guided Wave Letters, August 1998, vol. 8, no. 8.

[46] Schnieder, F., et al., "High Impedance Coplanar Waveguides with Low Attenuation", IEEE Microwave and Guided Wave Letters, March 1996, Vol. 6, No. 3., pp.117-119

[47] Jeong, S., et al., "Elevated-CPW for High-Speed Digital Interconnects," Antenna and Propagation Socity International symposium, 2001, pp. 541-544.

[48] Hwang, Y-J., et al., "Design of an Elevated CPW for High Speed Digital Circuit Applications," Antenna and Propagation Society International Symp., IEEE, vol. 1, 2001, pp. 588-591.

[49] Hettak, K., et al., "Design and Characterization of Elevated CPW and Thin Film Midrostrip Structures for Millimeter-Wave Applications", 2005 European Microwave Conference, vol. 2, Oct. 2005.

[50] Hofschen, S., "Simulation of an Elevated Coplanar Waveguide Using 2-D FDTD," IEEE Microwave and Guided Wave Letters, vol. 6, no. 1, 1996, pp. 28-30.

[51] Kamitsuna, H., "A Very Small, Low-Loss MMIC Rat-Race Hybrid Using Elevated Coplanar Waveguides," IEEE Microwave and Guided Wave Letters, vol. 2, no. 8, 1992, pp. 337- 339. 
[52] Lee, S-N., "Design and Characterization of Low-Loss and Low Dispersion Micromachined Elevated CPW," Microwave and Optical Technology Letters, vol. 39, no. 3, 2003, pp. 169-171.

[53] Reichelt, A., et al., "New Coplanar-Like Transmission Lines for Applications in Monolithic Integrated Millimeter-Wave and Submillimeter-wave Circuits," 1998 IEEE MTT-S Digest. Pp. 99-102.

[54] Kim, H-T, et al., "A New Microwmachined Overlap CPW Structure with Low Attenuation over Wide Impedance Ranges," 2000 IEEE MTT-S Digest. pp. 299-302.

[55] Milanovic, V., et al., "Characterization of Broad-Band Transmission for Coplanar Waveguides on CMOS Silicon Substrate," IEEE Trans. on MTT, vol. 46, no. 5, 1998, pp. 632-640.

[56] Eisenstadt, W.R, et al., "S-parameter based IC interconnect transmission line characterization," IEEE Trans. on Components, Hybrids, and Manufacturing Tech., vol. 15, no. 4, 1992, pp. 483-490.

[57] Williams, D., et al., "Accurate Transmission Line Characterization," IEEE Microwave and Guided Wave Letters, vol. 3, no. 8, 1993. pp. 247-249.

[58] Mangan, A., "De-Embedding Transmission Line Measurements for Accurate Modeling of IC Designs," IEEE Trans. on Electronic Devices, 2006, vol. 53, no. 2, pp. 235-241.

[59] Haroun, I., et al., "Experimental Study of WLAN 802.11b Over Optical Fiber", European Microwave Conference 2003, Munich, Germany, October 2003.

[60] Haroun, I., Gouin, F., "Measured Results for WLAN 802.11a Over Optical Fiber Radio System", $7^{\text {th }}$ Wireless World Research Forum (WWRF7), Eindhoven, The Netherlands, December 2002.

[61] Al-Raweshidy, H., Komaki, S., Radio over Fiber Technologies for Mobile Communications Network, Norwood, MA, Artech House, 2002.

[62] Kuri, Toshiaki, et al., "60-GHz-Band Full-Duplex Radio-On-Fiber System Using Two-RF-Port Electroabsorption Transceiver," IEEE Photoics Technology Letters, vol. 12, no. 4, pp. 419-421, Apr. 2000.

[63] Don, C.H, et al., "Millimeter-Wave CMOS Design," Trans. on JSSC, vol. 40, no. 1,2005 , pp. 144- 155 . 
[64] Don, C.H, et al., "Design Considerations for $60 \mathrm{GHz}$ CMOS Radios," IEEE Communications Magazine, December 2004, pp. 132-140.

[65] Don, C.H, et al., "Design of CMOS for $60 \mathrm{GHz}$ Applications," IEEE Int. Solid-State Circuits Conference, 2004, Session 24.

[66] Heydari, B., et al., "Low-Power mm-Wave Components up to $104 \mathrm{GHz}$ in 90nm CMOS", 2007 International Solid-State Circuits Conf., 2007, pp. 200201.

[67] Khanpour, M., "A Wideband W-Band Receiver Front-End in 65-nm CMOS," IEEE Trans. on JSSC, vol. 43, no. 8, 2008, pp. 1717-1730.

[68] Babakhani, A., "A 77-GHz Phased-Array Transceiver With On-Chip Antennas in Silicon: Receiver and Antennas," IEEE Trans. on JSSC, vol. 41, no. 12,2006 , pp. $2795-2806$.

[69] Yao, T., et al., "Algorithmic Design of CMOS LNAs and PAs for $60 \mathrm{GHz}$ Radio", IEEE Journal of Solid State Circuits, Vol. 42, No. 5, May 2007, pp. 1044- 1057.

[70] Lo, C-M., "A Miniature V-band 3-Stage LNA in 0.13um CMOS," IEEE Int. Solid-State Circuit Conf., 2006, Session 17.

[71] Sayag, A., "A 25 3.3 dB NF Low Noise Amplifier based upon Slow Wave Transmission Lines and the 0.18um CMOS Technology," IEEE RF Integrated Circuits Symposium, 2008, pp. 373-487.

[72] Cohen, E., et al., “ An ultra low power LNA with $15 \mathrm{~dB}$ gain and $4.4 \mathrm{~dB} \mathrm{NF}$ in $90 \mathrm{hm}$ CMOS process for $60 \mathrm{GHz}$ phase array radio", IEEE Radio Frequency Integrated Circuit Symposium, 2008, pp. 61-64.

[73] Pellerano, S., et al., "A $64 \mathrm{GHz}$ LNA with $15.5 \mathrm{~dB}$ Gain and $6.5 \mathrm{NF}$ in $90 \mathrm{~nm}$ CMOS", IEEE Journal of Solid State Circuit, vol. 43, no. 7, 2008, pp. 15421552 .

[74] Severino, R., et al., "A transformer-based 60GHz CMOS LNA for low voltage applications," IEEE RF Integration Technology, 2007, pp. 62-65.

[75] Kimberley, W., et al., "Compact Planar Microstrip Branch-Line and Rat-Race Couplers," IEEE Trans. on MTT, vol. 51, no. 10, 2003, pp. 2119-2125.

[76] Wang, J., et al., "A compact Slow-Wave Microstrip Branch-Line Coupler with High Performance," IEEE Microwave and Wireless Components Letters, vol. 17 , no. 7, 2007, pp. $501-503$. 
[77] Sun, K-O., et al., "A Compact Branch-Line Coupler Using Discontinuous Microstrip Lines," IEEE Microwave and Wireless Components Letters, vol. 15 , no. 8, 2005, pp. $519-520$.

[78] Lin, C-T., et al., "Finite-Ground Coplanar-Waveguide Branch-Line Couplers," IEEE Microwave and Wireless Components Letters, vol. 11, no. 3, 2001, pp. 127-129.

[79] Johnosono, S., et al., "Design of Broadband CPW Branch-Line 3-dB Couplers "Proceedings of the $36^{\text {th }}$ European Microwave Conference, 2006, pp. 36-39.

[80] Ozgur, M., et al., "Micromachined Branch Line Coupler in CMOS Technology ," 2000 IEEE MTT-S Digest, pp.291-294.

[81] Fujii, T., et al., "Miniature Broad-Band CPW Branch-Line Couplers in SlowWave Structure," IEICE Trans. Electron., vol. E90-C, no. 12, 2007, pp. 22452235.

[82] Yun, T-S., et al., "Millimeter Wave Branch Line Coupler Using Metal-AirMetal Capacitors ," Japanese Journal of Applied Physics, vol. 44, no. 2005, pp. 371-372.

[83] Lim, J., et al., " Design of $10 \mathrm{~dB} 90^{\circ}$ branch line coupler using microstrip line with defected ground," Electronics Letters, October 2000, vol. 36, no. 21, pp. 1784- 1785 .

[84] Reid, J., et al., "Micromachined Rectangular-Coaxial Transmission Lines ," IEEE Trans. on MTT, vol. 54, no. 8, 2006, pp. 3433-3441.

[85] Chong, T., "A Low-Noise, High-Linearity Balanced Amplifier in Enhancement-mode GaAS pHMET Technology for Wireless Base-Stations," $13^{\text {th }}$ GAAS Symposium, 2005, pp. 461- 464.

[86] Floyd, B., "V-Band and W-Band Low Noise Amplifiers and VoltageControlled Oscillator," IEEE Radio Frequency Integrated Circuits Symposium, 2004, pp. 295-298.

[87] Jin, J., et al., "A 1-V 45-GHz Balanced Amplifier With 21.5-dB Gain Using 0.18-um CMOS Technology," IEEE Trans. on MTT, vol. 56, no. 3, 2008, pp.599-603.

[88] Sharoury, F., et al., "The Design of Low LO-Power $60-\mathrm{GHz}$ CMOS Quadrature-Balanced Self-Switching Current-Mode Mixer," IEEE Microwave and Wireless Components Letters, vol. 18, no. 10, 2008, pp.692-694. 
[89] Hirota, T., et al., "Reduced-Size Branch-Line and Rat-Race Hybrids for Uniplanar MMIC's, "IEEE Trans. on MTT, vol. 38, no. 3, 1990, pp. 270-275.

[90] He, J., et al., "A Compact Microstrip Barnch-Line Coupler with Capacitor Loading," IEEE MTT-S, 2008, pp. 139-141.

[91] Hettak, K., et al., "Compact MMIC CPW and Asymmetric CPS Branch-Line Couplers and Wilkinson Dividers Using Shunt and Series Stub Loading," IEEE Trans. on MTT, vol. 53, no. 5, 2005, pp. 1624- 1635.

[92] Ponchak, G.E., "Experimental Analysis of Reduced-Sized Coplanar Waveguide Transmission Lines," IEEE MTT-S Digest, 2003, pp. 971-974.

[93] Scardelletti, M., et al., "Miniaturized Wilkinson Power Dividers Utilizing Capacitive Loading," IEEE Microwave and Wireless Components Letters, vol. 12 , no. 1,2002 , pp. 6-8.

[94] Singh. R., et al., "Miniaturized $20 \mathrm{GHz}$ CPW Quadrature Coupler using Capacitive Loading," Microwave and Optical Technology Letters, vol. 30, no. 1, 2001, pp.3-5.

[95] Reid, R.J., et al., "A $60 \mathrm{GHz}$ Branch Line Coupler Fabricated Using Integrated Rectangular Coaxial Lines," IEEE MTT-S Disgest, 2004, pp. 441444.

[96] Amaya, R.E., et, al., "A $60 \mathrm{GHz}$ CMOS Balanced Downconversion Mixer with a Layout Efficient $90^{\circ}$ Hybrid Coupler ," IEEE Custom Integrated Circuits Conference (CICC), 2009, pp. 235-238.

[97] Haroun, I., Wight, J., Plett, C., Fathy, A., Chang, D.,"Experimental Analysis of a $60-\mathrm{GHz}$ Compact EC-CPW Branch-Line Coupler for mm-Wave CMOS Radios," accepted for publication in IEEE Microwave and Wireless Components Letters. Manuscript Number: P03431.

[98] Haroun, I., Wight, J., Plett, C., Fathy, A,“" Experimental Characterization of EC-CPW Transmission Lines and Passive Components for $60-\mathrm{GHz}$ CMOS Radios," accepted for publication in IEEE MTT 2010 International Microwave Symposium, May 23-28, 2010. 